EXPERIMENTAL INVESTIGATION OF A 2-D AIR AUGMENTED ROCKET:

HIGH PRESSURE RATIO AND TRANSIENT FLOW-FIELDS

\author{
A Thesis \\ Presented to \\ The Faculty of California Polytechnic State University, \\ San Luis Obispo
}

\author{
In Partial Fulfillment \\ Of the Requirements for the Degree of \\ Master of Science in Aerospace Engineering
}

\author{
By \\ Josef Salvador Sanchez \\ March 2012
}


(C) 2012

Josef Salvador Sanchez

ALL RIGHTS RESERVED 
TITLE:

AUTHOR:

DATE SUBMITTED:

COMMITTEE CHAIR:

COMMITTEE MEMBER:

COMMITTEE MEMBER:

COMMITTEE MEMBER:
Experimental Investigation of a 2-D Air Augmented Rocket: A Study of High Pressure Ratios and Transient Flow-fields

Josef Salvador Sanchez

March 2012
Dr. Dianne DeTurris, Aerospace Engineering Dept.

Dr. David Marshall, Aerospace Engineering Dept.

Dr. Joseph Mello, Mechanical Engineering Dept.

D. Ryan Gist, Pratt \& Whitney Rocketdyne 


\section{ABSTRACT \\ Experimental Investigation of a 2-D Air Augmented Rocket: \\ High Pressure Ratio and Transient Flow-fields \\ Josef Salvador Sanchez}

A 2-D Air Augmented Rocket, the Cal Poly Air Augmented Rocket (CPAAR) Test Apparatus operating as a mixer-ejector was tested to investigate high stagnation pressure ratio and transient flow fields of an ejector. The primary rocket ejector was supplied with high pressure nitrogen at a maximum chamber pressure of 1758 psia and a maximum mass flow rate of $1.4 \mathrm{lb} / \mathrm{s}$. The secondary flow air was entrained from a fixed volume plenum chamber producing pressures as low as 3.3 psia. The maximum total pressure ratio achieved was 221. The original CPAAR apparatus was rebuilt re-instrumented and capability expanded. A fixed volume plenum was attached to the secondary ducts through a constant area square section to mimic the cross section of the secondary ducts with a bell mouth inlet. The mixing duct length was increased from 8 in. to 18 in.

An investigation of the mixing duct flow-field was done with data from pressure and temperature instrumentation. A study of the transient operation of the rocket was compared with results from former research to qualify the quasi-steady assumption of the flow-field. The CPAAR produced Fabri-choked operation, the startup transient observed caused the secondary flow to become established during Fabri-choke mode operation. The supersonic saturated mode was not observed during quasi-steady operation. The quasi-steady operation was defined based on characteristics from previous quasi-steady models of transient operation of supersonic ejectors.

The measurement of the data during testing resulted in a $2.96 \%$ experimental uncertainty in the entrainment ratio calculation. The smallest entrainment ratio observed was 0.05 at a total pressure ratio of 220. The location of the Fabri-choke point was shown through the interpretation of the primary and secondary flow as a result of the pressure and temperature measurements. The experimental evidence showed the location of the secondary choke point has a logarithmic relationship with the total pressure ratio. At a total pressure ratio of 220, the area of the aerodynamic throat of the secondary flow is 0.26 in $^{2}$ and the location occurs 6 inches downstream from the nozzle exit. The secondary flow un-choke is related to the breakdown of the shock structure of the primary flow and produces a flow-field asymmetry which blocks the right duct flow.

The CPSE simulation was unable to accurately predict AAR performance when the inputs are changed from the original CPAAR configuration. At high pressure ratios $(\mathrm{PR}=220)$, the error in the prediction is $90 \%$. 


\section{ACKNOWLEDGMENTS}

I would like to thank my advisor Dr. Dianne DeTurris. Without her guidance, knowledge, and enthusiasm I could not have had the degree of success which I have experienced in my educational career thus far. I need to give special thanks to Cody Thompson of the Aerospace Engineering Department for the knowledge and support he offered me. The experience I received while working as his assistant provided me with the tools, literally and figuratively, to complete this research.

I must thank Ryan Gist, Trevor Foster and Brett Morham for the initial work and basis of this research. Martin Popish and Trevor Montre for their work on this project, which without, this research could not have been completed.

Without the help and guidance I received from my family and friends I would be lost. My parents, Maria and Jose, have continually inspired me and supported me throughout all of my efforts. My grandparents, Ines and Vern, have offered an invaluable amount of guidance and support in my pursuit of education. My aunt and uncle, Socorro and Tom, have treated me like a son and have always offered a helping hand when I needed it. My brothers, Niko and Mikael, have always been a source of inspiration and motivation for success. I would also like to thank all of my friends who helped me step back, relax, and come back to my work with fresh ideas and motivation. Daniella Castro for her perpetual support, Romel Auza and Jason Baltierrez for their friendship and inspiration to be a better and harder working individual. And for the mutual support in our pursuit of graduate education, Mike Podevin, who is witness to the amount of time and energy spent on this work. 


\section{TABLE OF CONTENTS}

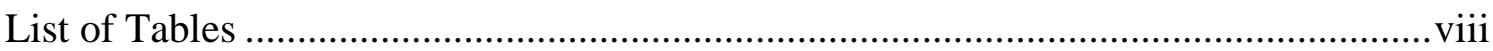

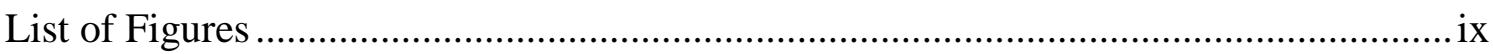

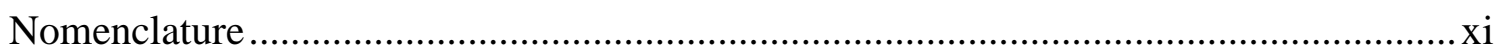

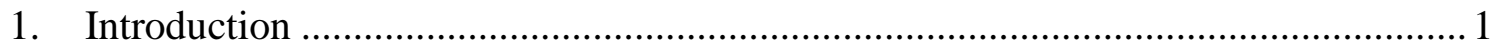

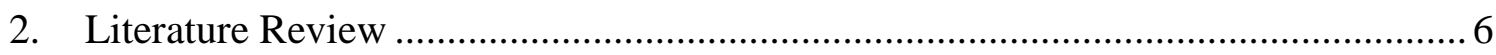

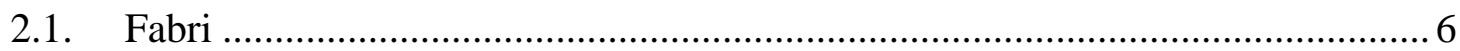

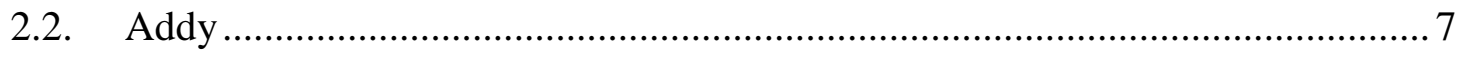

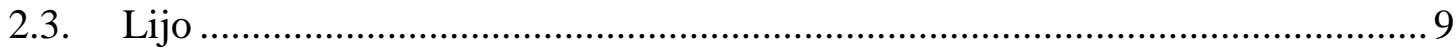

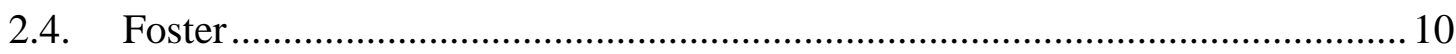

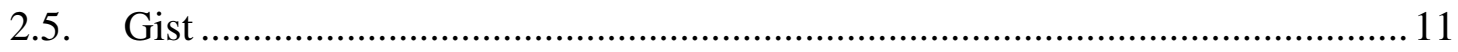

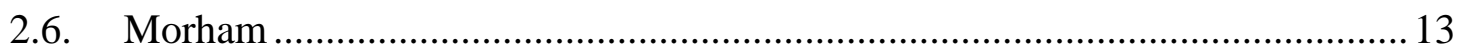

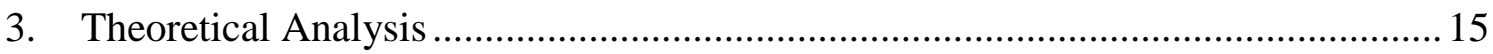

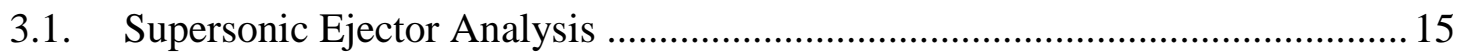

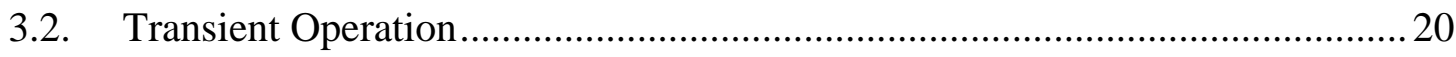

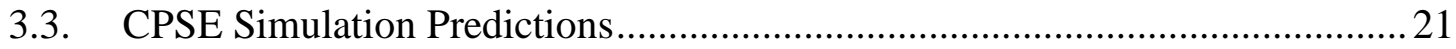

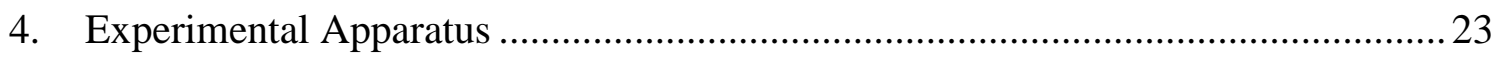

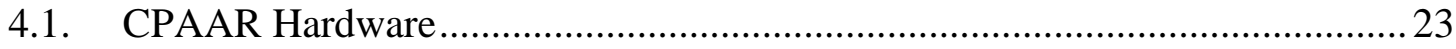

4.1.1. Air Augmented Rocket ...................................................................... 24

4.1.2. Secondary Duct Vacuum Plenum .........................................................2

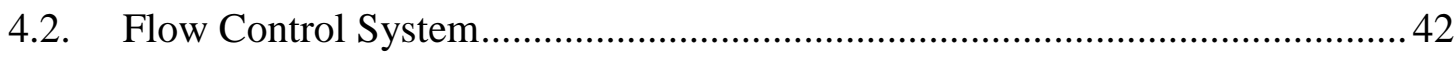

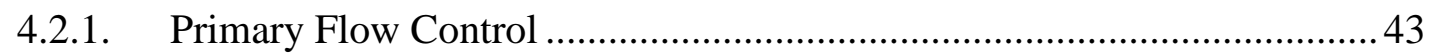

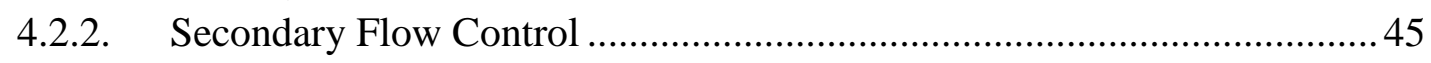

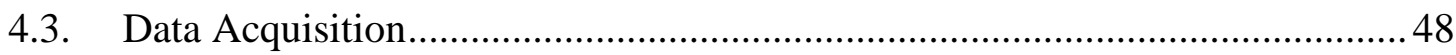

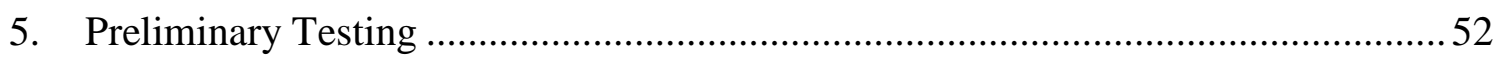

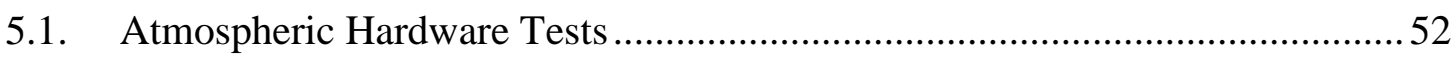

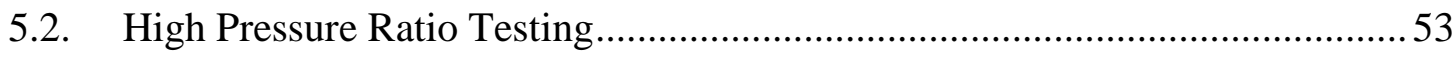

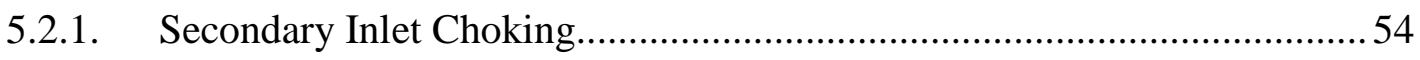

5.2.2. Secondary Inlet Constant Area Duct Configuration .................................55

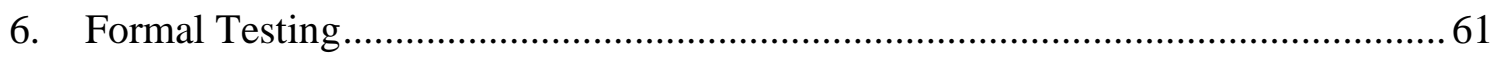

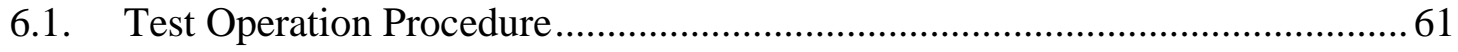

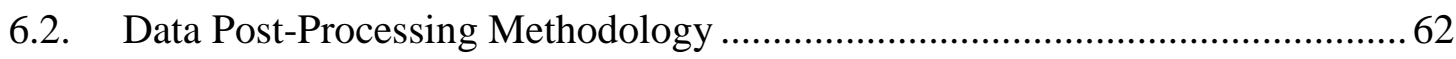

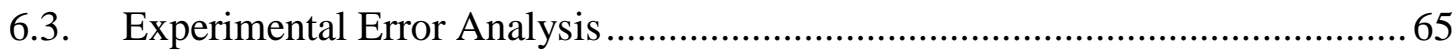

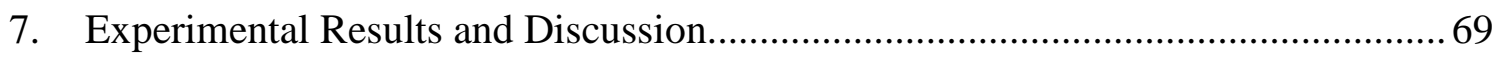

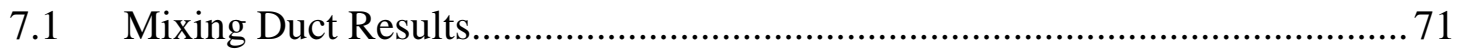

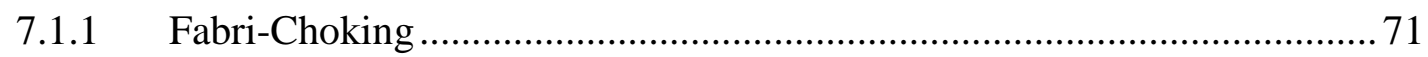

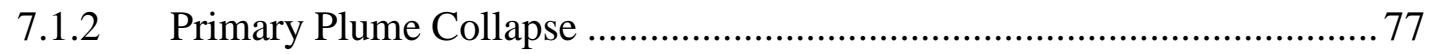

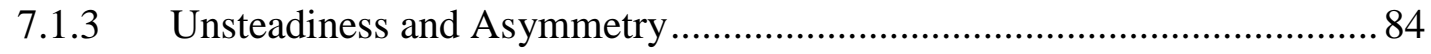

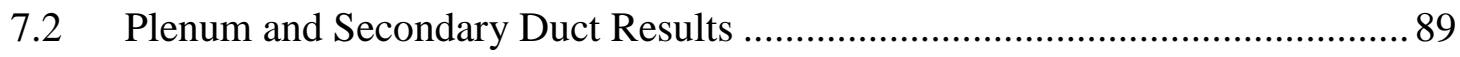

7.2.1 Secondary Flow Measurement Differences ............................................ 89 


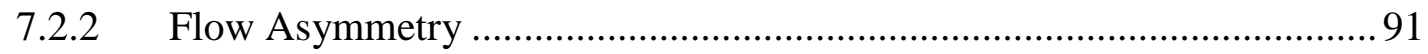

7.2.3 Plenum Quasi-Steady Assumption ..................................................... 92

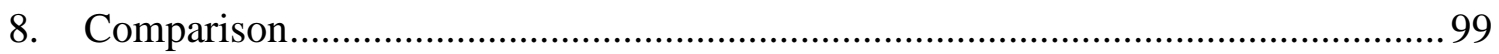

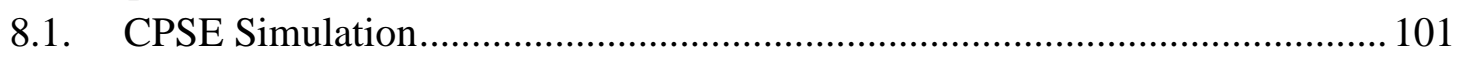

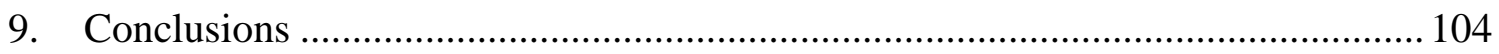

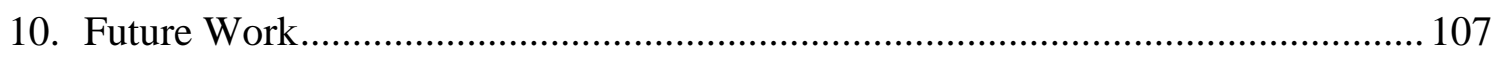

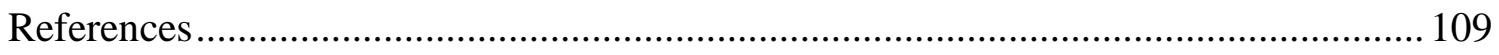

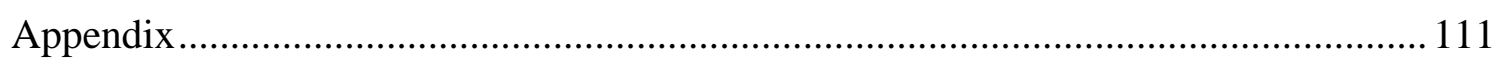




\section{List of Tables}

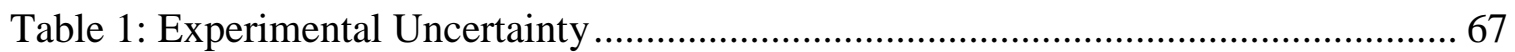

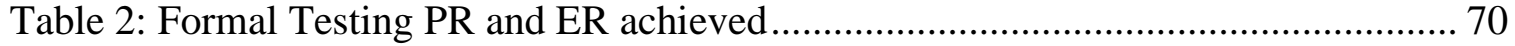

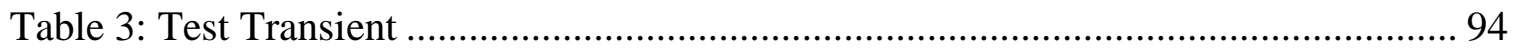

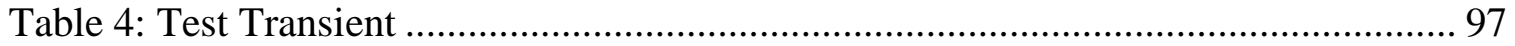

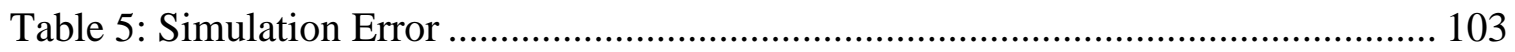




\section{List of Figures}

Figure 1: Propulsion Efficiency of High Speed Propulsion Engine Cycles ${ }^{4}$..................... 3

Figure 2: Air-Augmented Rocket Schematic ${ }^{4}$............................................................. 4

Figure 3: Addy's Transient Flow Process ${ }^{7}$.................................................................... 8

Figure 4: Variation between ideal and actual primary plume expansion ${ }^{4}$......................... 12

Figure 5: Primary plume growth, ideal to actual size due to empirical correction factor ${ }^{9} 12$

Figure 6: CPSE code compared to Gist experimental data and empirical predictions ${ }^{11}$.. 14

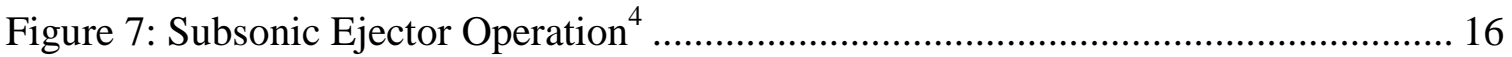

Figure 8: Supersonic Mixed Ejector Operation ${ }^{4}$....................................................... 17

Figure 9: Supersonic Ejector Operation, Saturated Mode ............................................ 18

Figure 10: Supersonic Ejector Operation, Fabri-choke Mode ....................................... 18

Figure 11: Supersonic Ejector Operation, Fabri-Block Mode ........................................ 19

Figure 12: Transient Flow Region ${ }^{7}$.............................................................................. 21

Figure 13: CPSE, PR = 250, Static Pressure Distribution of the blocked flow pattern ${ }^{11} .22$

Figure 14: CPAAR Test Apparatus (Not to Scale) .......................................................... 24

Figure 15: Model of the Cal Poly Supersonic Ejector ${ }^{10}$.............................................. 25

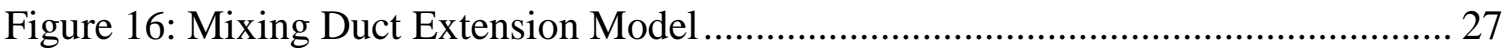

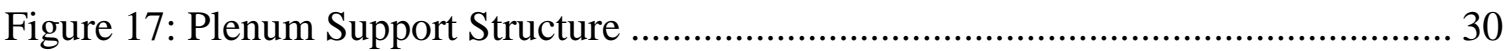

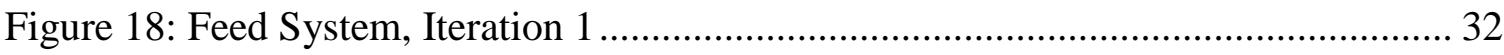

Figure 19: Rocket Manifold Feed System Inlet, Iteration 2 ...................................... 33

Figure 20: Secondary Inlet Area Differences Diagram, Iteration 2 ............................... 34

Figure 21: Secondary Duct, Plenum to Inlet Connection ............................................. 35

Figure 22: Flow Path for Inlet Configuration Iteration 3............................................. 36

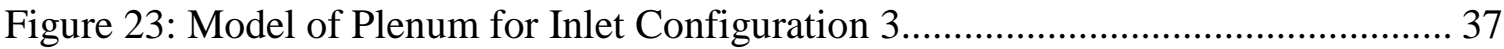

Figure 24: Secondary Duct Configuration, Inlet Horn and Inlet Extension ..................... 38

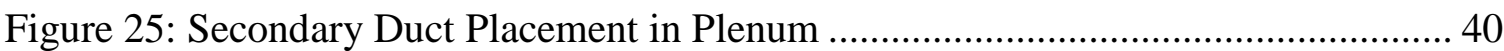

Figure 26: Support Plate with Secondary and Primary Inlets Installed, Side View ......... 41

Figure 27: Interface Plate with Secondary and Primary Inlets Installed, Top View ........ 42

Figure 28: Primary Flow Pressurization System ....................................................... 43

Figure 29: Primary Flow Pressurization System .......................................................... 44

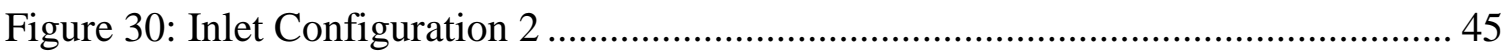

Figure 31: Relief System, Inlet Configuration 3........................................................ 46

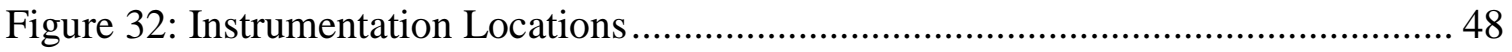

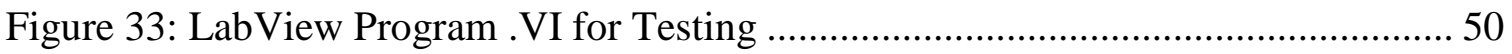

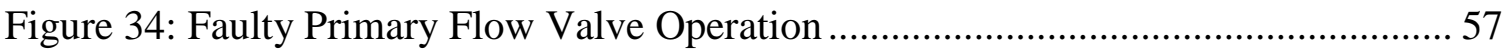

Figure 35: Normal Primary Flow Valve Operation ..................................................... 57

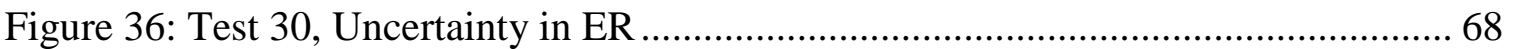

Figure 37: Test 31, Secondary Flow Mach number.................................................. 72

Figure 38: Test 31, Mach Numbers along Right Mixing Duct Flow-field ...................... 73

Figure 39: Test 30, Mach number vs. Location .......................................................... 74 
Figure 40: Wall Location of Secondary Fabri-choke Point ........................................... 75

Figure 41: Fabri-choke Location and Size................................................................ 76

Figure 42: Mixing Duct Centerline Mach number, Test 33........................................... 78

Figure 43: Centerline Static Pressure, Test 33........................................................... 79

Figure 44: Mixing Duct Wall Static Pressure, Test 33 ............................................... 80

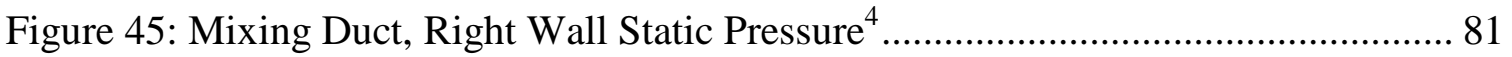

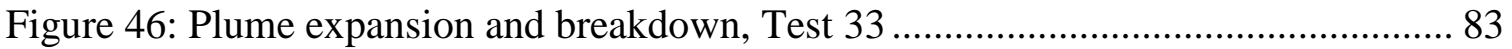

Figure 47: Test 33, Secondary Mach number and Total Pressure Ratio .......................... 84

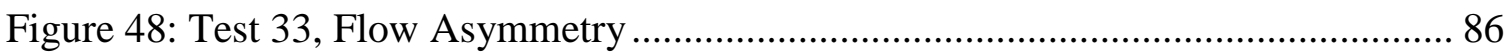

Figure 49: Test 30, Mixing Duct Right Wall Mach.................................................... 87

Figure 50: Test 30, Ejector Performance and Right Duct Block ................................... 88

Figure 51: Test 31, Secondary Inlet, Left Duct Stagnation Pressure Loss ...................... 90

Figure 52: Test 31, Secondary Duct Asymmetry....................................................... 92

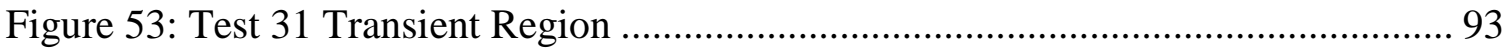

Figure 54: Test 31, Total Transient Region .............................................................. 95

Figure 55: Test 31, Maximum Pressure Ratio during Working Transient Region ........... 96

Figure 56: Test 31, Secondary Mach number and Total Pressure Ratio ......................... 97

Figure 57: Test 33, Secondary Mach number and Total Pressure Ratio ......................... 98

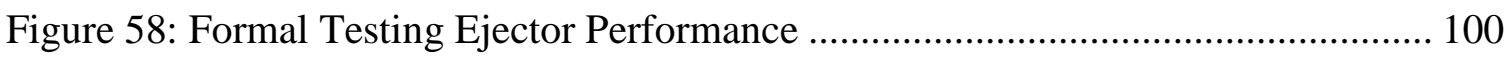

Figure 59: Ejector Performance and CPSE Simulation .............................................. 102 


\section{Nomenclature}

\begin{tabular}{|c|c|c|}
\hline A & Area & $\left(\mathrm{ft}^{2}, \mathrm{in}^{2}\right)$ \\
\hline $\mathrm{a}$ & Speed of Sound & $(\mathrm{ft} / \mathrm{s})$ \\
\hline $\mathrm{E}$ & Young's Modulus & $\left(\mathrm{lb}_{\mathrm{f}} / \mathrm{in}^{2}\right)$ \\
\hline ER & Entrainment Ratio $=\dot{m}_{S} / \dot{m}_{P}$ & - \\
\hline F & Force & $\left(\mathrm{lb}_{\mathrm{f}}\right)$ \\
\hline I & Area Moment of Inertia & $\left(\mathrm{in}^{4}\right)$ \\
\hline $\mathrm{K}$ & Column Effective Length Factor & - \\
\hline $\mathrm{L}$ & Length & (in) \\
\hline M & Mach Number & - \\
\hline $\mathrm{M}_{\mathrm{c}}$ & Critical Mach Number $=\left(V_{p}-V_{s}\right) /\left(a_{p}-a_{s}\right)$ & - \\
\hline$\dot{m}$ & Mass Flow Rate & $\left(\mathrm{lb}_{\mathrm{m}} / \mathrm{s}\right)$ \\
\hline OD & Outer Diameter & (in) \\
\hline $\mathrm{P}$ & Stagnation Pressure & $\left(\mathrm{lb}_{\mathrm{f}} / \mathrm{in}^{2}\right)$ \\
\hline $\mathrm{p}$ & Static Pressure & $\left(\mathrm{lb}_{\mathrm{f}} / \mathrm{in}^{2}\right)$ \\
\hline PR & Stagnation Pressure Ratio $=\mathrm{P}_{0 \mathrm{p}} / \mathrm{P}_{0 \mathrm{~s}}$ & \\
\hline psi,psia & Absolute Pressure & $\left(\mathrm{lb}_{\mathrm{f}} / \mathrm{in}^{2}\right)$ \\
\hline psig & Gauge Pressure & $\left(\mathrm{lb}_{\mathrm{f}} / \mathrm{in}^{2}\right)$ \\
\hline $\mathrm{R}$ & Universal Gas Constant & $\left(\mathrm{lb}_{\mathrm{f}} \mathrm{ft} / \mathrm{lb}_{\mathrm{m}}{ }^{\circ} \mathrm{R}\right.$ \\
\hline $\mathrm{T}$ & Temperature & $\left({ }^{\circ} \mathrm{F},{ }^{\circ} \mathrm{R}\right)$ \\
\hline $\mathrm{t}$ & Time & (s) \\
\hline th & Thickness & (in) \\
\hline V & Velocity & $(\mathrm{ft} / \mathrm{s})$ \\
\hline $\mathrm{x}$ & Distance Downstream of Nozzle Lip & (in) \\
\hline \multicolumn{3}{|l|}{ Greek } \\
\hline$\gamma$ & Ratio of Specific Heats & - \\
\hline$\varepsilon$ & Nozzle Expansion Ratio $=A_{\mathrm{e}} / \mathrm{A}^{*}$ & - \\
\hline$\mu$ & Dynamic Viscosity & $\left(\operatorname{slug} / \mathrm{ft}^{*} \mathrm{~s}\right)$ \\
\hline$v$ & Poisson’s Ratio & - \\
\hline$\rho$ & Density & $\left(\operatorname{slug} / \mathrm{ft}^{3}\right)$ \\
\hline$\sigma$ & $1^{\text {st }}$ Standard Deviation & - \\
\hline
\end{tabular}

\section{Subscripts}

0

c Critical

e Nozzle Exit

i Upstream of Choking

p, 1 Primary Stream

s, 2 Secondary Stream

$\mathrm{v} \quad$ Plenum

\section{Superscripts}

* $\quad$ Critical Point (sonic throat condition) 


\section{Introduction}

For over a century, engineers have researched, designed and developed propulsion systems for powered flight. The success of the Wright brothers' first powered flight can be heavily attributed to their development of a research facility capable of studying airfoil shapes in a controlled laboratory setting, the wind tunnel. The father of modern rocketry, Robert $\mathrm{H}$. Goddard would perform static engine tests, after the development of or change to a new design of engine before laboratory tests. ${ }^{1}$

The ability to test theories developed by scientists and engineers is essential to the scientific method and the development of new technologies. By testing scaled down models of systems, time and cost are able to be drastically reduced on research and development projects. The difficulty lies in being able to develop a facility that can properly mimic the operational conditions of the system. Being able to accurately reproduce those conditions is essential to the success of the research and development phase of any project.

The process of testing in a controlled situation has been used since the beginning of modern aerospace development. But at a certain point, there is only so much static or small scale testing that can be done to prove a theory of flight or performance. A full scale test must be performed, and have systems which allow us to understand that the test being conducted proves or disproves understood theories. Some of the finest examples of this in aerospace are the $\mathrm{X}$-planes that have been developed and tested throughout history.

The North American X-15 was powered by a single XLR99 rocket engine capable of propelling the vehicle above Mach 6 . The X-15 still holds the speed record for manned 
rocket-powered flight ${ }^{2}$. The X-15 test series produced an incredible amount of scientific data about flight at hypersonic speeds and continues to be an inspiration for spaceplane development.

The $\mathrm{X}-15$, despite its performance capabilities, shares the main disadvantage associated with rocket-powered vehicles, the necessity of carrying large quantities of propellant, fuel and oxidizer, increasing weight and reducing range. Research done in the area of combined cycle engines, try to use the advantages of different engine types and combine them into a single system.

Combined cycle engines have been an area of research for the past 50 years in the aerospace industry. A combined cycle engine has many advantages over a single cycle engine such as a rocket, turbojet or ramjet. A classic example of a combined cycle engine is the J-58 propulsion system of the Lockheed SR-71 Blackbird ${ }^{3}$.

The $\mathrm{J}-58$ is a combined cycle turbo-ramjet engine. At low speeds the engine operates as a typical turbojet engine and at high speeds the engine transitions to ramjet operation. This allows the Blackbird to operate continuously at speeds that afterburning turbojets are incapable of sustaining. This gives the vehicle an advantage in propulsive efficiency over range of speed when compared to other types of propulsion systems as Figure 1 presents. 


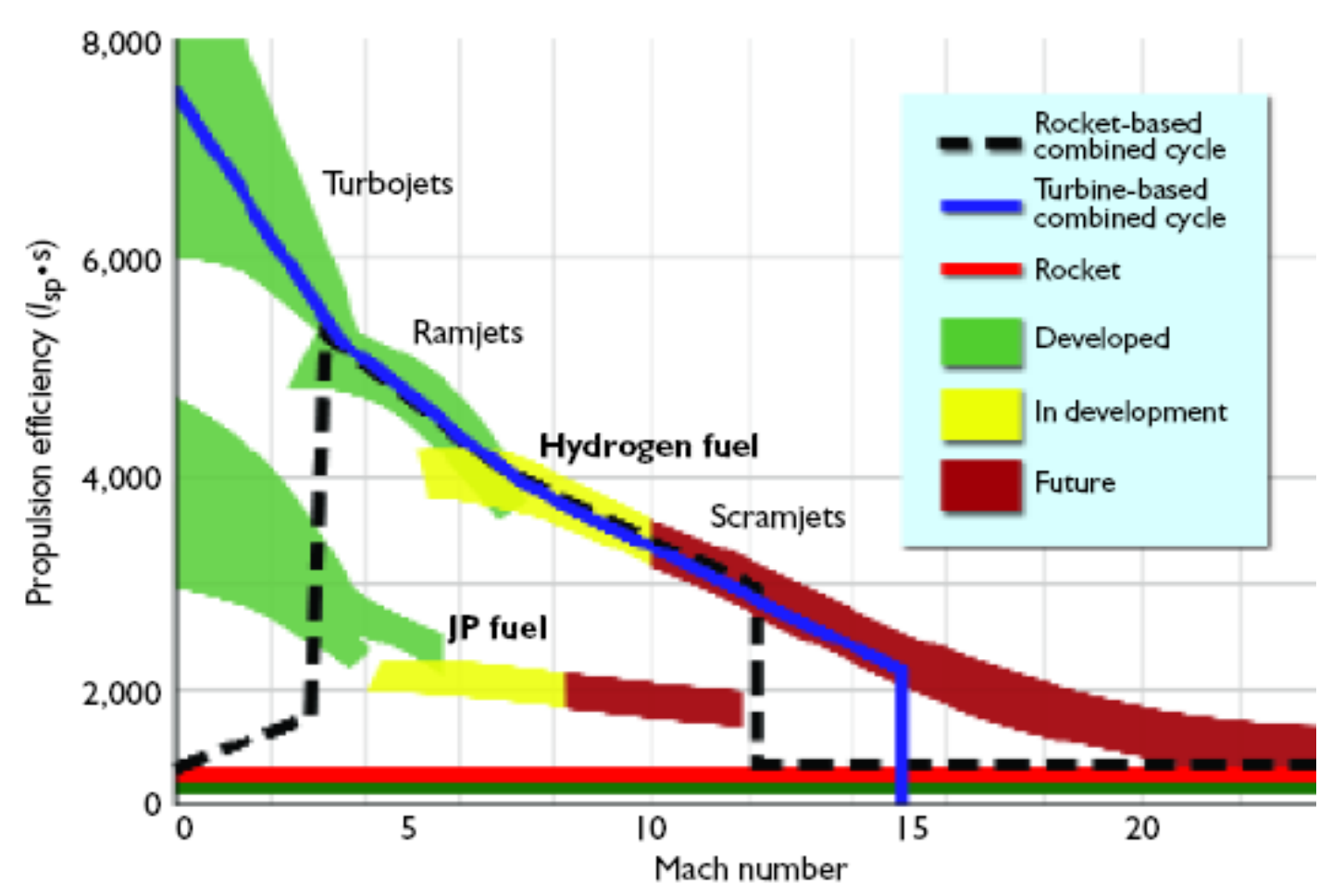

Figure 1: Propulsion Efficiency of High Speed Propulsion Engine Cycles ${ }^{4}$

A rocket based combined cycle engine has several clear advantages over other forms of high speed propulsion. It offers the high thrust take off capability of a rocket, and the high speed efficiency and capability of a ram or scramjet. By utilizing an air-breathing mode, the amount of oxidizer required for the aircraft to carry onboard is drastically reduced. As a technology still under significant amounts of research, the development of hardware capable of reproducing several operation conditions is required.

The first operational mode of a Rocket Based Combined Cycle (RBCC) engine is the AirAugmented Rocket (AAR) mode. This mode is used to propel the vehicle from take off to supersonic speeds such that the engine can be operated in its ramjet mode.

A Rocket-Based Combined Cycle engine is a type of hypersonic air-breathing engine that is capable of propelling a vehicle into orbit without the assistance of multiple stages, a Single Stage to Orbit (SSTO) operation. The RBCC engine operates with several 
distinct modes to achieve orbital insertion, an air-augmented rocket take off, ejectorramjet, pure ramjet, scramjet, and pure rocket. By utilizing different modes of operation, the system can utilize each mode during a period of flight when its efficiency is the highest.

Over the past six years research at Cal Poly San Luis Obispo has been conducted with the goal of understanding the development of flow in a supersonic ejector. The research has focused on the development and operation of hardware capable of operating as a supersonic ejector in cold and hot flow conditions simulating the operation of an AirAugmented Rocket (AAR), as shown in Figure 2.

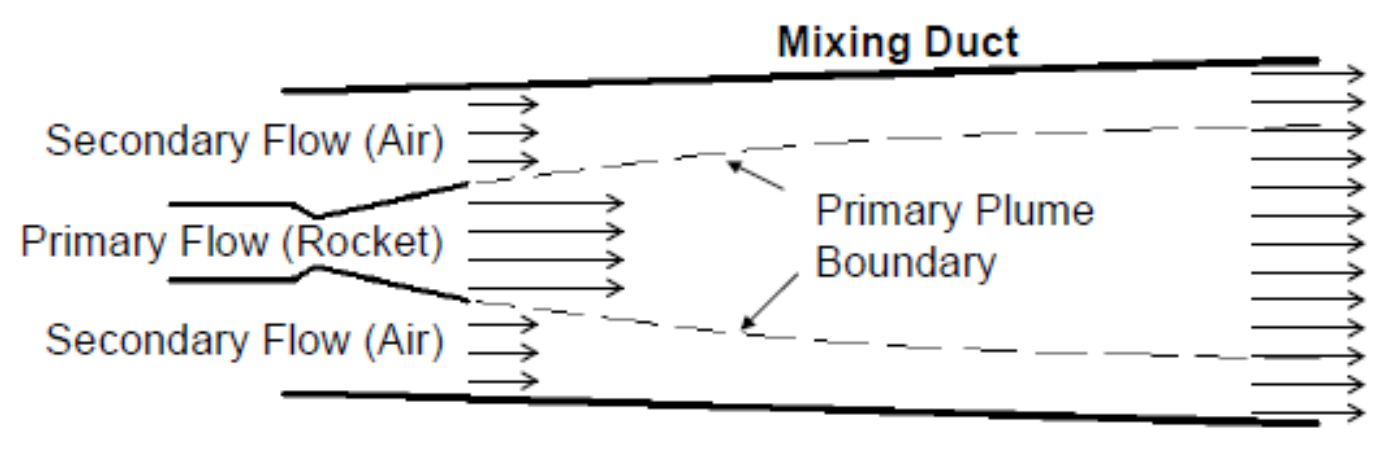

Figure 2: Air-Augmented Rocket Schematic ${ }^{4}$

The Air-Augmented Rocket system is, when simplified, similar to an ejector. An ejector is a device composed of two separate flows that converge to produce a mixed flow. In an ejector the primary flow is the flow which has, relative to the secondary flow, significantly higher momentum. The primary flow entrains a lower momentum secondary flow into an area where the two flows interact. The area where the two flows interact is called the "mixing duct." In terms of an Air-Augmented Rocket system, the primary flow is considered the rocket exhaust, and the secondary flow is an inlet exposed to 
atmospheric flow, the mixing duct would then be a secondary chamber where the two flows can interact.

The goal of this research was to expand the capabilities of the existing experimental apparatus and understand the change in performance compared to expected results. The expansion of capabilities was done by designing, building and operating a plenum chamber connected to the secondary inlets of the Cal Poly Air-Augmented Rocket (CPAAR) to reduce the stagnation pressure of the secondary flow and artificially influence the interaction of the primary and secondary flows. The results of the tests were compared to previous research by Foster and Gist, as well as theoretical predictions produced by Morham’s Cal Poly Supersonic Ejector Simulation code.

In current experiments as well as previous experiments by Foster and Gist, the pressure ratio is not constant, due to a simultaneously changing secondary pressure and primary chamber pressure. The primary chamber energizes to a maximum in a very quick transient operation. This quick change initiates the decrease in pressure of the plenum. The rate of evacuation is at its highest during this point and then begins to decrease as a function of the decreasing primary chamber pressure. 


\section{Literature Review}

The works used to provide information for the analysis and development of the current research were from authors who defined characteristics of general ejector flow and authors of the previous research done at Cal Poly.

Fabri defined the operation of air to air ejectors. Addy went into further detail of supersonic air to air ejectors, by studying axisymmetric flow with viscous and transient analysis. Lijo studied the operation of an ejector in a vacuum operation and used computational fluid dynamics to model and understand the flow conditions and assumptions at critical operational points.

The design, development and initial analysis of the Cal Poly Air-Augmented Rocket (CPAAR) were done by Gist and Foster. A subsequent simulation code, the Cal Poly Supersonic Ejector (CPSE) Simulation code was developed by Morham and produced predictions of the CPAAR operation based on experimental data from Gist and Foster.

\section{1. $\quad$ Fabri}

Fabri et al. ${ }^{5,6}$ presents the first investigation of a supersonic air to air ejector. An axisymmetric ejector with a supersonic primary and subsonic secondary flow was studied. The analysis done by Fabri does not take into account viscous interactions between the low supersonic primary flow and subsonic secondary flow, but does use a correction for pressure loss due to friction between the secondary flow and the duct wall. The flow at the exit of the mixing duct is uniform and equal to ambient conditions.

To interpret the flow field in ejector operation, Fabri defines the flow with relation to the flow velocity at the exit of the mixing duct: the subsonic case, saturated supersonic, and 
Fabri-choke supersonic case. In these cases the flow at the mixing duct is considered uniform. In the case that the mixing duct is too short, insufficient mixing of the two flows can produce a non-uniform flow at the exit consisting of a supersonic primary and subsonic secondary with an identifiable slip line, called the mixed case.

Fabri concludes that his inviscid analysis is valid for supersonic ejector flow-fields with similar specific heat ratios, high Mach numbers and high Reynolds numbers. He concludes that the flow field is maintained by primarily inviscid forces and viscous effects are not significant in the interaction of the two flows.

\subsection{Addy}

$\operatorname{Addy}^{7}$ presented a model to study the flow phenomenon of supersonic air-to-air axisymmetric ejectors. The assumptions of his model are analogous to Fabri's assumptions of similar flow composition, supersonic primary, subsonic or sonic secondary flow, and a uniform flow at the exit of the mixing duct. To improve upon the Quasi-1D model proposed by Fabri, Addy suggests a 2-D model based on the method of characteristics to model the supersonic primary plume and a 1-D model for the secondary flow. The solution is based on an iterative method which assumes a pressure matched boundary condition between the two flows. Addy also suggests using a viscous superposition method to model the viscous interaction of the flows based on the inviscid solution.

Addy also discusses the topic of transient operation based on characteristic times. The characteristic time is based on flow and ejector geometry. Addy suggests that based on characteristics times it is justifiable to analyze transient ejector problems on a quasisteady basis. The transient flow can be considered quasi-steady if the time required to 
establish the secondary flow is considered to be negligible in comparison with the overall process time ${ }^{7}$. He breaks the transient flow process into two regimes, the starting, and quasi-steady regimes. He characterizes the transient flow process as quasi-steady if the starting regime is about $1 \%$ of the overall process time. The characterization is done by comparing the entrainment ratio as a function of the test time as represented in Figure 3.

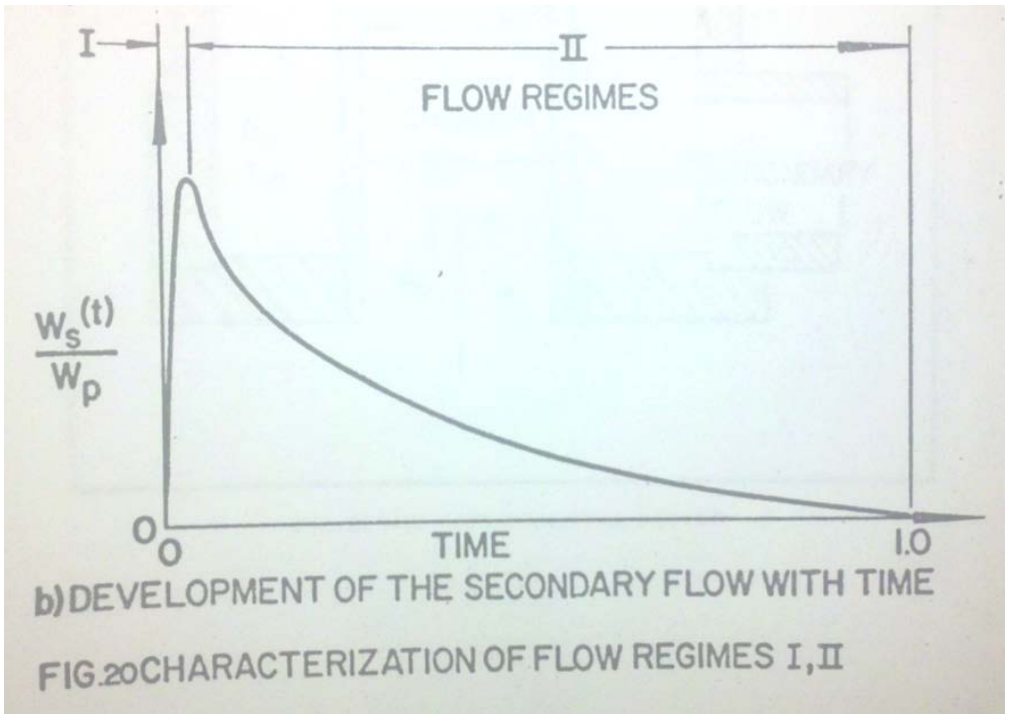

Figure 3: Addy's Transient Flow Process ${ }^{7}$

Figure 3 shows the two flow regimes which Addy describes. The first flow regime is the startup regime where the secondary flow becomes established to a peak mass flow rate and decreases during the second flow regime, the quasi-steady state regime, to a base value over a characteristic time of the flow. The characteristic flow is dependent on the starting and establishment of the secondary flow.

The flow mechanism which governs the majority of mass transfer between the two flows is the viscous mixing at small secondary flow mass rates ${ }^{7}$. Addy develops this method because the steady state ejector theory developed by Fabri was inadequate at low 
secondary mass flow rates when viscous mixing is the primary method of mass transfer between the two flows.

He concludes that the correlations between his analytical and available experimental results are "indistinguishable" for transient conditions and acceptable for steady-state conditions.

\subsection{Lijo}

$\mathrm{Lijo}^{8}$, with a multinational team of researchers studied the transient flow of a vacuum ejector system with the help of computational fluid dynamics. The team attempted to investigate the phenomenon of continuous entrainment into the primary stream with a limited mass supply from the secondary chamber. The team studied a supersonic primary flow which interacted with a subsonic secondary flow connected to a fixed volume chamber. The investigation observed the transient region of the ejector operation and characterized the formation of the primary plume and boundary layer through CFD software. In the model, the two flows are separated by a thin lip before the flows interact.

The results showed that continuous mass entrainment can be possible in this configuration of ejector systems when a recirculation zone near the primary nozzle exit is created. The recirculation zone is created after the transient region of operation and the flow in the secondary chamber achieves a state of dynamic equilibrium of pressure. At the dynamic equilibrium point, the static pressure in the secondary chamber remains constant while the primary flow continues to operate within its design parameters. The phenomenon of continuous mass entrainment after this point is created by the formation of the recirculation zone at the nozzle lip. The steady state assumption can only be applied to the flow field after the point of dynamic equilibrium. 
The current study of flow phenomena of the CPAAR was developed from the recommendations of previous research on the subject. The apparatus was developed and studied initially by two former Cal Poly Aerospace Engineering graduate students, Ryan Gist ('07) and Trevor Foster ('08). Several series of tests, including hot and cold flow were conducted to observe the characteristics of the developed system and compared to theoretical model results. Based on those initial series of tests a simulation code was developed by Brett Morham ('10) to be able to predict the performance of the system with given inlet conditions of the system.

\section{4. $\quad$ Foster}

Trevor Foster's research ${ }^{9}$ on supersonic ejectors was done with his experimentation with the Cal Poly Air Augmented Rocket. Foster developed the CPAAR, a two-dimensional supersonic ejector, simulating the operation of an Air Augmented Rocket. The primary flow of the system was combusted Methane and Gaseous Oxygen. The secondary flow was standard air entrained from atmosphere. The objective of the study was to observe the interaction of the primary plume with the entrained secondary flow and to observe theorized Fabri conditions for the CPAAR application. The hot fire tests were conducted after an extended test series of cold flow high pressure nitrogen tests.

The configuration of the CPAAR was based on a design suggested by Aerojet and the X43B program. The intent was to experimentally verify initial studies they had conducted

on the adverse affects on secondary entrainment by an air drop launch condition for a ducted rocket. The launch condition in question was an altitude of approximately 38,000 ft with a Mach number of $0.7^{9}$. 
The tests were conducted with primary chamber pressures ranging from 325 psi to 1032 psi with an oxidizer to fuel mixture ratio of 2 . For the duration of this test series the Methane Oxygen Combustion achieved a maximum pressure ratio of 74 . This was not enough to create a Fabri-choke condition in the mixing duct. Foster observed the location of the secondary flow restriction in the mixing duct was independent of the primary flow chamber pressure and temperature. The observation of a constant secondary flow area demonstrates the inability of the primary flow to cause a reduction in entrainment of the secondary flow at the achieved pressure ratio. The testing verified the saturated case of supersonic ejector operation.

\subsection{Gist}

While using the hardware developed by Foster, Gist conducted a series of cold flow tests with high pressure nitrogen to obtain higher pressure ratios than those obtained during hot fire tests ${ }^{4,10}$. The objective was to produce tests which exhibited Fabri-choking and Fabri-block conditions of the supersonic ejector system. He used nitrogen for the primary flow and entrained air from atmosphere, using the assumption that the two flows have equal gas constants. To achieve a Fabri-choke condition in the mixing duct, Gist modified the apparatus set up by Foster to operate in a cold flow configuration. The primary flow was connected to a high pressure gaseous nitrogen system, which produced primary flow chamber pressures up to 1690 psi. Gist's prediction of the plume size was based on 1-D isentropic assumptions, and attempted to visually verify the size of the flow. The actual flow was predicted to be larger due to the shock structure of the primary plume which is not accounted for in ideal calculations, Figure 4. 

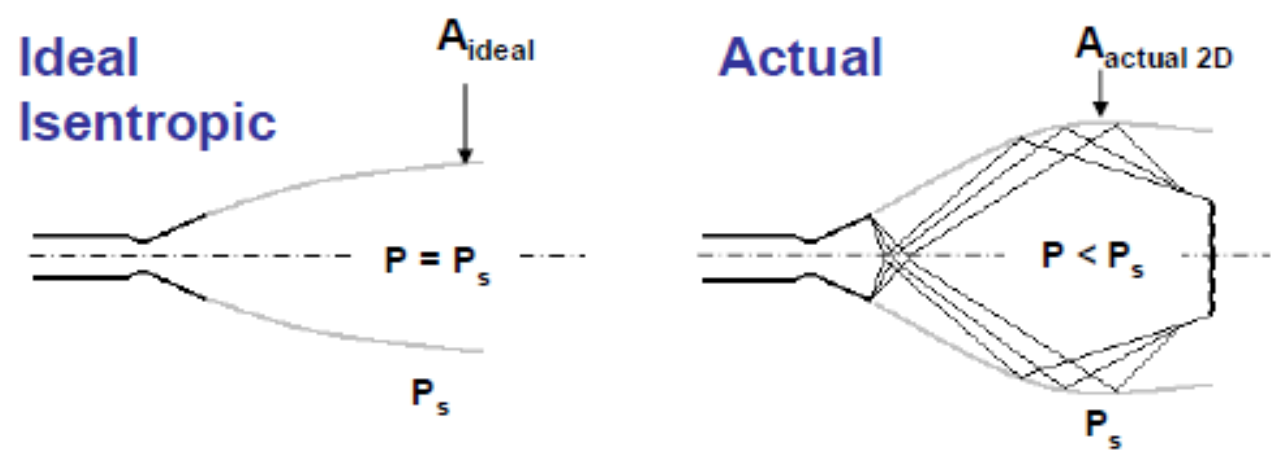

Figure 4: Variation between ideal and actual primary plume expansion ${ }^{4}$

Gist was able to visualize the shock structure of the primary flow during supersonic operation. He was able to capture the oblique shocks emanating off the lip of the nozzle on video to demonstrate the increase in plume area due to non-isentropic expansion. To theoretically predict the entrainment ratio of the system he used a one dimensional isentropic approximation developed by Fabri visually approximated in Figure 5.

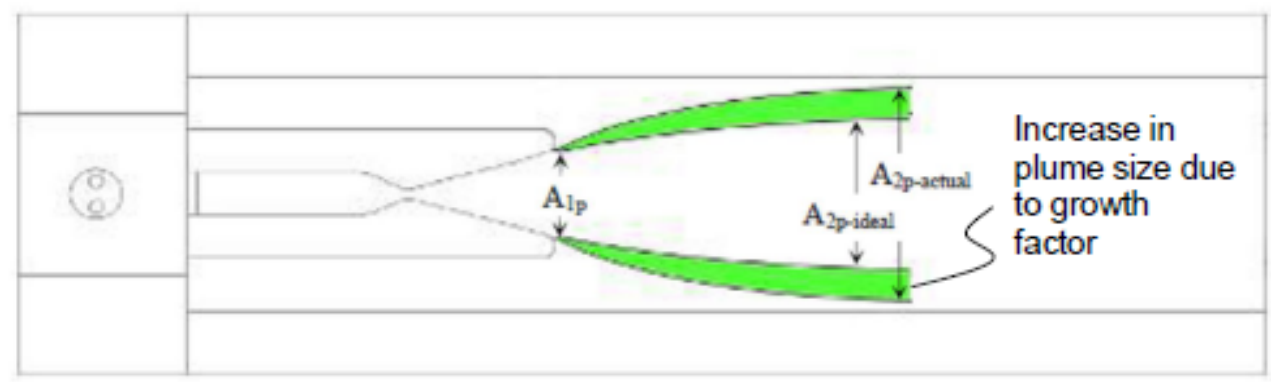

Figure 5: Primary plume growth, ideal to actual size due to empirical correction factor ${ }^{9}$

To account for the increased plume size due to the shock structure of the primary plume, Gist used an empirical correction factor, k-expand. The correction factor improves prediction of entrainment during the Fabri-choke mode of operation in the ejector. The correction factor used to estimate the increased plume is determined from values which match experimental data. 


\subsection{Morham}

Brett Morham ${ }^{11}$ developed a MATLAB simulation code the approximate the flow development of the CPAAR.

"The purpose of the simulation is to identify the operating conditions which produce the saturated, Fabri-choke and Fabri-block aerodynamic flow patterns. The effect of primary to secondary stagnation pressure ratio on the efficiency of the ejector operation is measured using the entrainment ratio which is the secondary to primary mass flow ratio.” 11

"The primary flow of the ejector is supersonic and the secondary (entrained) stream enters the ejector at various velocities at or below Mach 1 . The primary and secondary streams are both composed of air. The primary plume boundary and properties are solved using the Method of Characteristics. The properties within the secondary stream are found using isentropic relations along with stagnation conditions and the shape of the primary plume. The solutions of the primary and secondary streams iterate on a pressure distribution of the secondary stream until a converged solution is attained. Viscous forces and thermo-chemical reactions are not considered."

Morham compared between the results produced by the simulation code he developed the experimental data obtained by Gist, and the 1-D analysis approximation with $\mathrm{k}_{\text {expand }}$ approximations. Figure 6 is a presentation of the accuracy of Morham's predictions to the experimental data and 1-D approximation. 


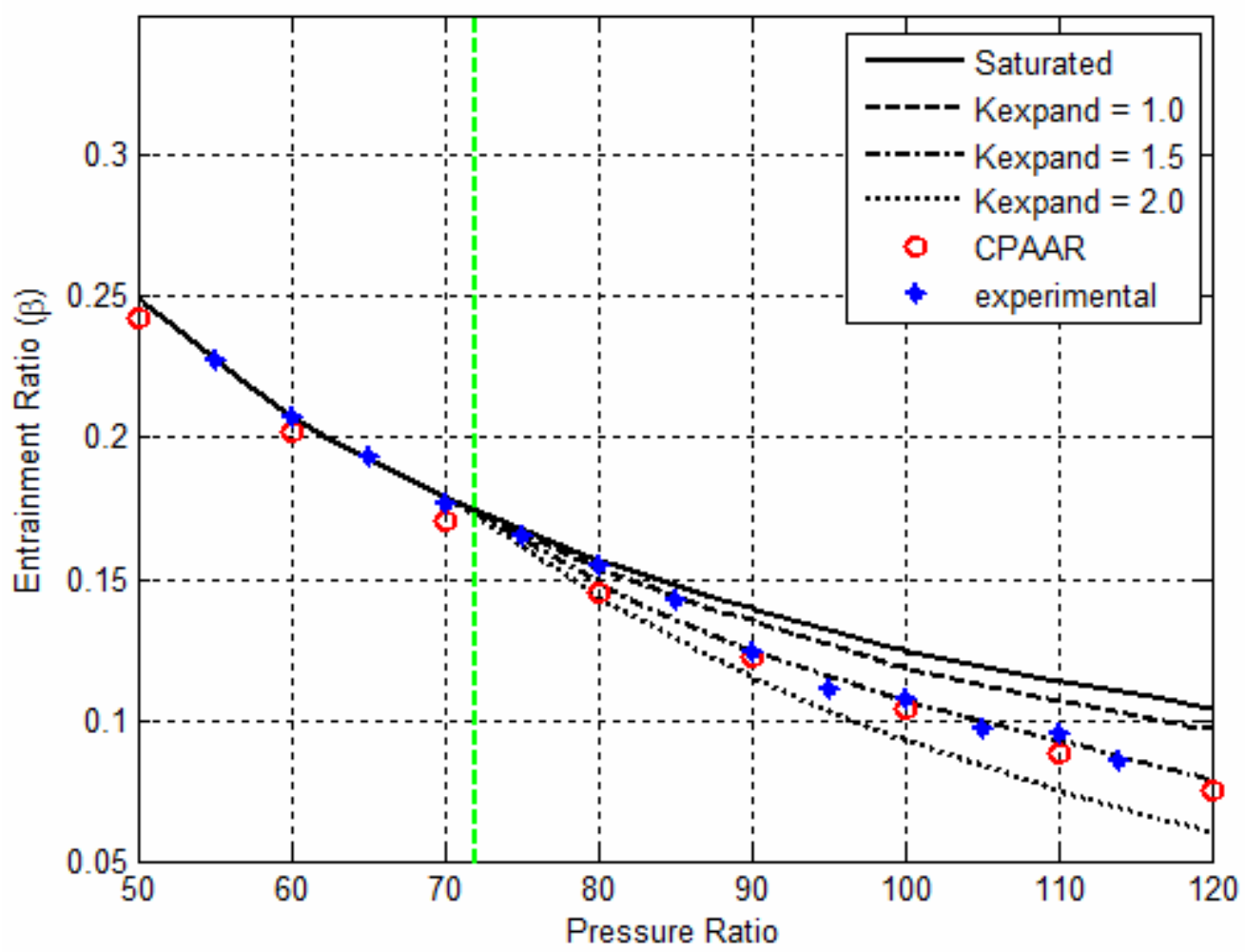

Figure 6: CPSE code compared to Gist experimental data and empirical predictions ${ }^{11}$

The simulation was based on the conditions of both flows at certain times during a test with the geometry data of the apparatus. Morham validated the saturated flow condition at pressure ratios below 74, as well as the Fabri-choke case at pressure ratios above 74 . The simulation had an average error of $3.57 \%$ relative to Gist's experimental data. Morham was also able to simulate up to a pressure ratio of 230, where the Fabri-block condition would theoretically occur. He concluded ejector operation is driven by pressure gradient effects rather than viscous effects. 


\section{Theoretical Analysis}

The current work uses theory similar to previous research on the apparatus with respect to supersonic-ejector analysis. In addition, the nature of the current research requires an analysis of the transient properties of the configuration. The objective of the research was to broaden the experimental range of the hardware and to produce data to be used as validation of a simulation code as well as validation of flow assumptions placed on transient flow. The theoretical analysis will describe the application of the assumptions to different flow conditions of a supersonic ejector.

\subsection{Supersonic Ejector Analysis}

As a subsonic compressible fluid flows into the converging section of a nozzle, the static pressure will decrease as the velocity increases. The properties of a supersonic nozzle state that at the location of minimum area, the flow has a Mach number of one. As the flow expands through the diverging section of the nozzle it will decrease in static pressure and increase in velocity until the exit.

An air-augmented rocket (AAR) operates on the same principles as an ejector. An ejector is a simple device where a primary high speed flow interacts with a secondary flow in a mixing duct. The secondary flow is entrained by the primary flow into the mixing duct. In the AAR, the high speed flow is the rocket exhaust and the secondary flow is entrained from inlets exposed to atmosphere.

The CPAAR configuration is a simulated AAR, operating as an ejector. An ejector has several distinct modes of operation which are dictated by the flow conditions at the inlet 
and exit of the mixing duct. The modes are identified by the condition of the flow at the exit of the mixing duct.

Subsonic operation of the ejector occurs when the primary flow pressure is low enough or the secondary flow and mixing duct exit pressure is high enough such that the primary plume is over-expanded and terminated in a normal shock as shown in Figure 7. The primary flow and secondary flow will mix and become subsonic. The flow at the exit of the mixing duct will be subsonic, and considered to be a fully mixed composition of the two flows. Full mixing of the two flows will only occur at the exit of the mixing duct when the mixing duct has a length to diameter ratio of ten ${ }^{12}$.

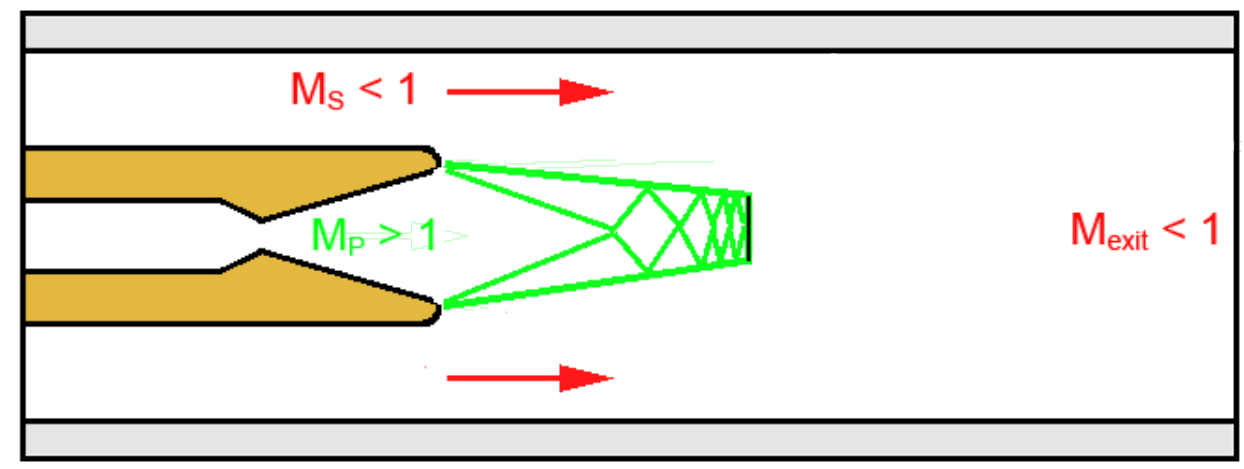

Figure 7: Subsonic Ejector Operation ${ }^{4}$

Ejectors can also operate in a mixed condition where, the supersonic primary pressure is greater than the previous condition but still slightly over-expanded. The resulting flow, shown in Figure 8 does not mix and the flow at exit of the mixing duct is composed of the subsonic secondary flow and supersonic primary flow. The entrainment of the secondary flow into the mixing duct is dependent on the conditions of the primary flow and at the exit of the mixing duct during subsonic and mixed ejector operation. 


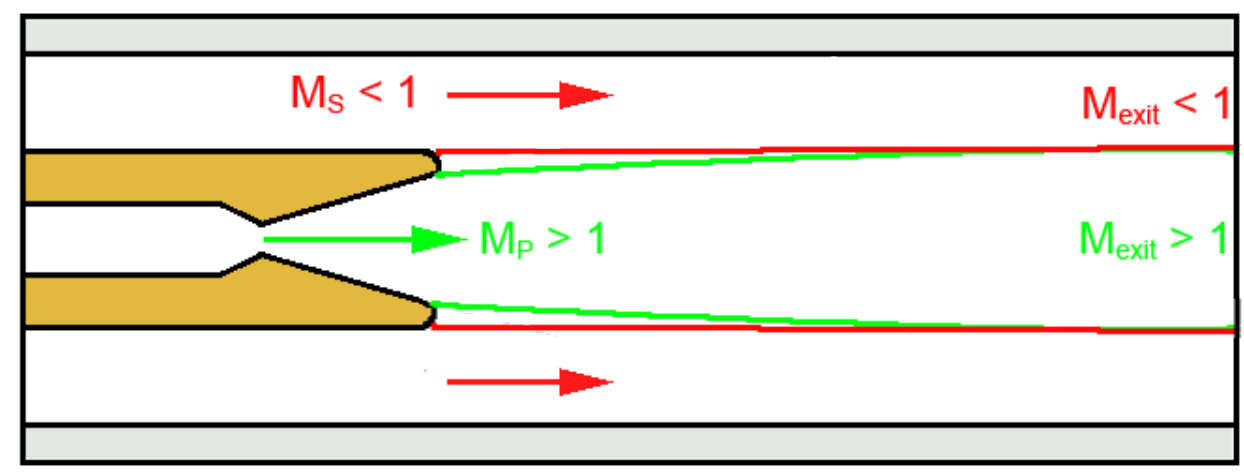

Figure 8: Supersonic Mixed Ejector Operation ${ }^{4}$

During supersonic operation, the secondary entrainment is independent of conditions at the mixing duct exit and only dependent on the primary to secondary pressure ratio, ejector geometry, and if the flow is choked ${ }^{12}$.

Supersonic operation of the ejector occurs when the primary flow pressure is large enough to cause under-expansion of the plume such that the plume boundary interferes with the flow path of the secondary flow. The reduction of flow area for the secondary causes the flow at the wall to accelerate to a sonic condition. The flow then begins to mix, and if the mixing duct is long enough, the flows are fully mixed and supersonic at the exit of the mixing duct.

The purpose of this research is to expand on the previous research of the supersonic case by Gist, Foster and Morham. During supersonic operation, the flow of an ejector can be classified once again by three distinct modes, first studied and subsequently named by and after Fabri ${ }^{5}$. 


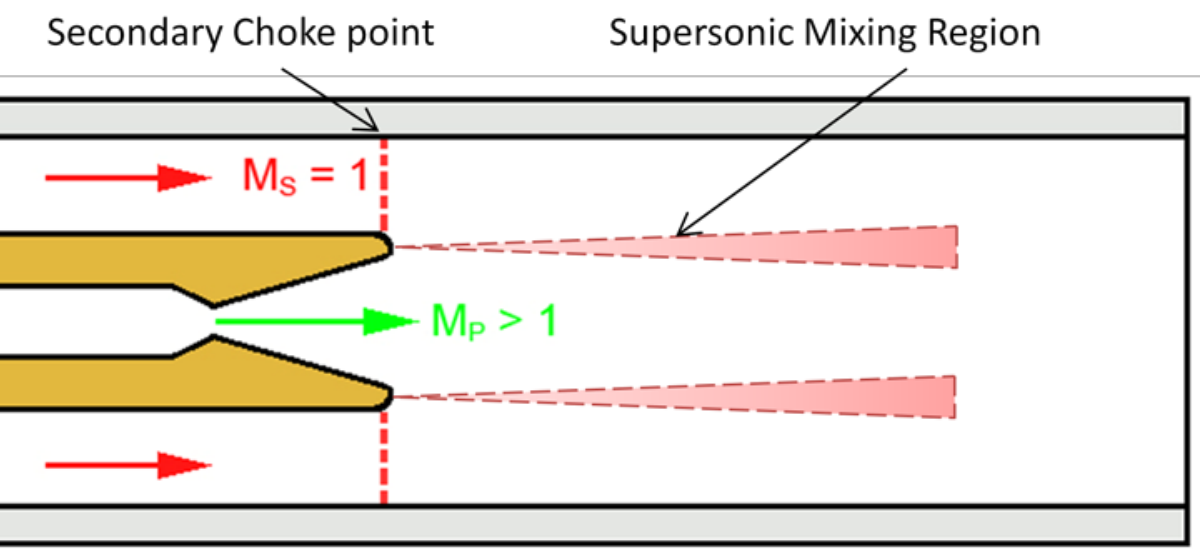

Figure 9: Supersonic Ejector Operation, Saturated Mode

The Fabri mode which is evident at the lowest primary to secondary stagnation pressure ratio of the supersonic condition is the saturated case. The saturated case contains a supersonic primary flow that is near or at its optimal expansion point. This case results in the secondary flow to become choked at the duct of the secondary flow, resulting in the maximum entrainment available during supersonic operation. Once the plume passes the point of optimal expansion, the plume begins to under-expand and begins to impinge on the secondary flow.

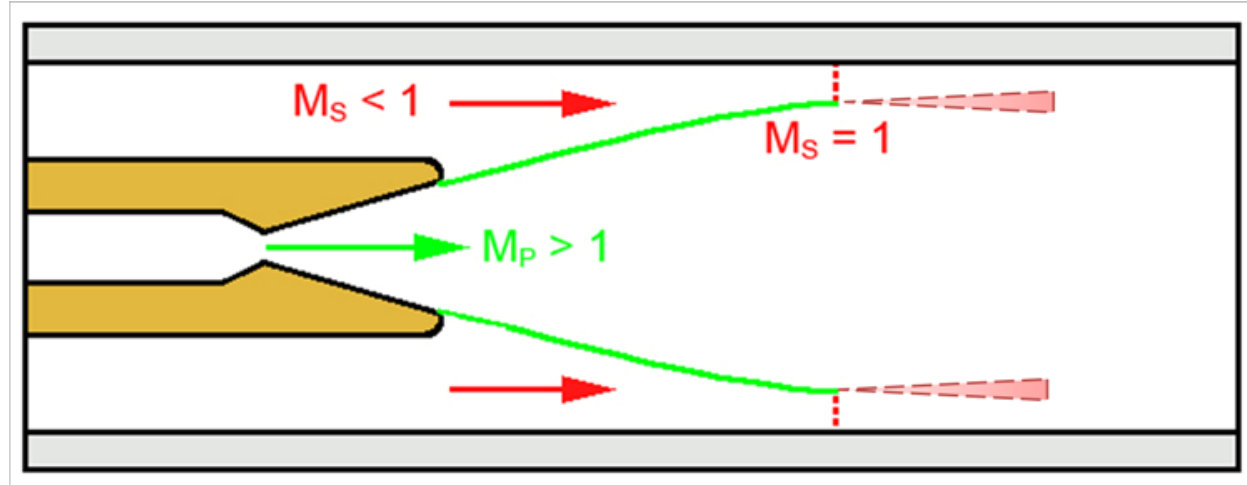

Figure 10: Supersonic Ejector Operation, Fabri-choke Mode

The Fabri-choke condition occurs when the supersonic primary plume has become underexpanded such that the minimum flow area of the secondary flow becomes reduced, resulting in a reduction in mass flow rate. At the sonic location of the secondary flow, 
mixing does not immediately occur, but the flow will become fully mixed with sufficient mixing duct length ${ }^{7}$.

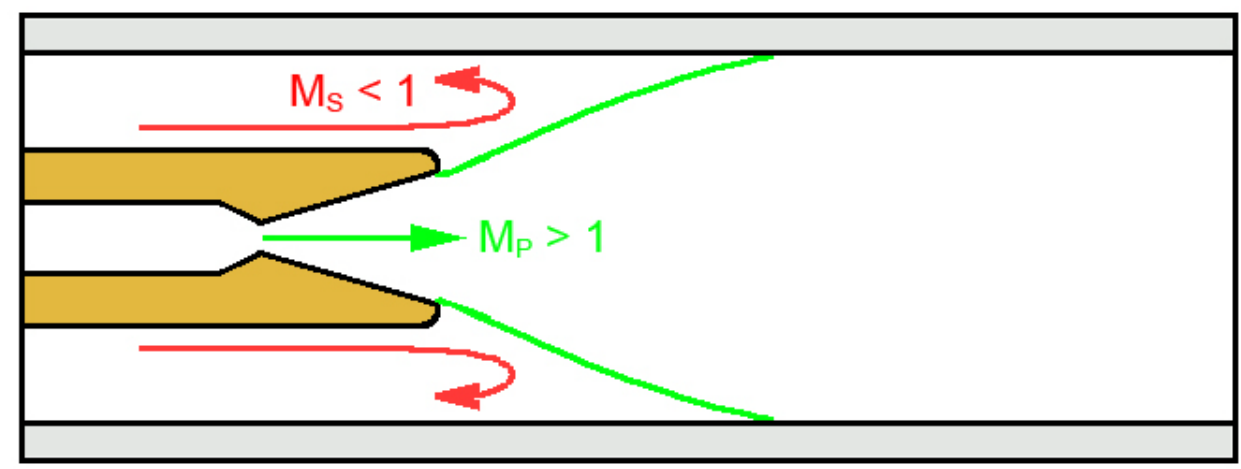

Figure 11: Supersonic Ejector Operation, Fabri-Block Mode

When the primary to secondary pressure ratio is large enough, the flow will become so under-expanded that it will impinge with the duct wall. The secondary flow will be unable to mix with the primary flow and at this point there will be no mass transfer from the secondary flow to the primary flow. The goal of this research is to study the entrainment of the secondary flow at pressure ratios near or at the Fabri-block condition. Previous research has demonstrated that the entrainment of the secondary flow into the mixing duct at high pressure ratios is governed by pressure distribution effects rather than viscous interactions of the flows ${ }^{11}$.

To quantify the efficiency of performance of supersonic ejectors, an entrainment ratio is evaluated. The entrainment ratio is the ratio of the secondary mass flow rate to the primary mass flow rate. The variable represents the composition of the flow in the mixing duct.

$$
E R=\frac{\dot{m}_{s}}{\dot{m}_{p}}
$$


The entrainment ratio is dependent on the stagnation pressure ratio, the ratio of the primary stagnation pressure to the secondary stagnation pressure.

$$
P R=\frac{P_{c}}{P_{S}}
$$

Evaluating these two parameters against each other produces an efficiency curve that represents the performance of the system. At pressure ratios above the saturated condition, the mass flow rate is reduced as a function of the under-expansion of the primary plume.

\subsection{Transient Operation}

The primary upgrade to the CPAAR is the addition of a fixed volume plenum attached to the secondary flow system. This means, as the primary flow entrains the secondary flow, the pressure inside the fixed volume plenum will decrease at a rate relative to that. This creates the condition where the flow is inherently transient throughout the entire operation. During operation, the pressure in the plenum will be reduced until a potential base pressure is achieved. But also, dependent on the primary flow, another transient parameter could be applied. Both Addy and Lijo have studied the application of the steady state assumption to a transient flow system. A steady state primary flow operates without change in the shock structure of the primary plume ${ }^{7,8}$.

The transient region during the operation of the CPAAR will occur from the start of the pressurization of the primary chamber until the minimum pressure in the plenum is achieved. This region is subsequently divided into two flow regimes ${ }^{7}$, the startup transient, and the quasi-steady transient regimes. Figure 12 shows the characteristic time of the transient flow region divided into the two regimes. 


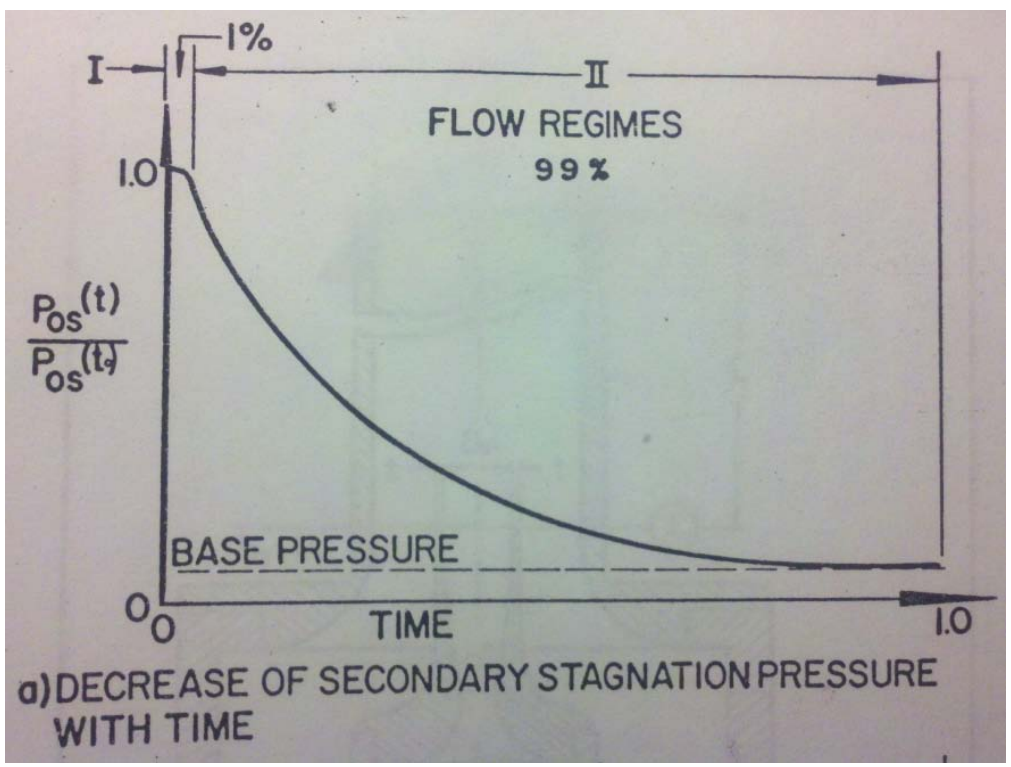

Figure 12: Transient Flow Region ${ }^{7}$

The startup transient begins at the pressurization of the primary flow, and ends when the secondary flow begins to entrain into the mixing duct. The time of the startup transient regime should be approximately $1-2 \%$ of the total transient region for the quasi-steady regime to be assumed as quasi-steady and be comparable to steady flow results ${ }^{7}$.

\subsection{CPSE Simulation Predictions}

The code requires geometry inputs of the system as well as raw data of the flow conditions of the primary and secondary flows. The data input into the simulation are derived from a data point of a test and are used to determine properties of the primary and secondary flows. The properties of interest are the size, shape and velocity of the primary plume, as well as the velocity and choke location and size of the secondary flow. The purpose of the present study is to produce experimental data to further verify the predictions of the CPSE simulation at high pressure ratios. 
The CPSE simulation code was used to predict the size of the primary plume and resulting mass flow rate dependent on conditions of the primary and secondary flows. Raw recorded data of the two flows will be used as inputs for the flow conditions of the simulation.

The code has been able to predict with a relative high accuracy the entrainment of the secondary flow. The code also predicted with the previous test set up, that a Fabri-block case would occur at pressure ratios of 230. A static pressure distribution of the two predicted flows is shown in Figure 13.

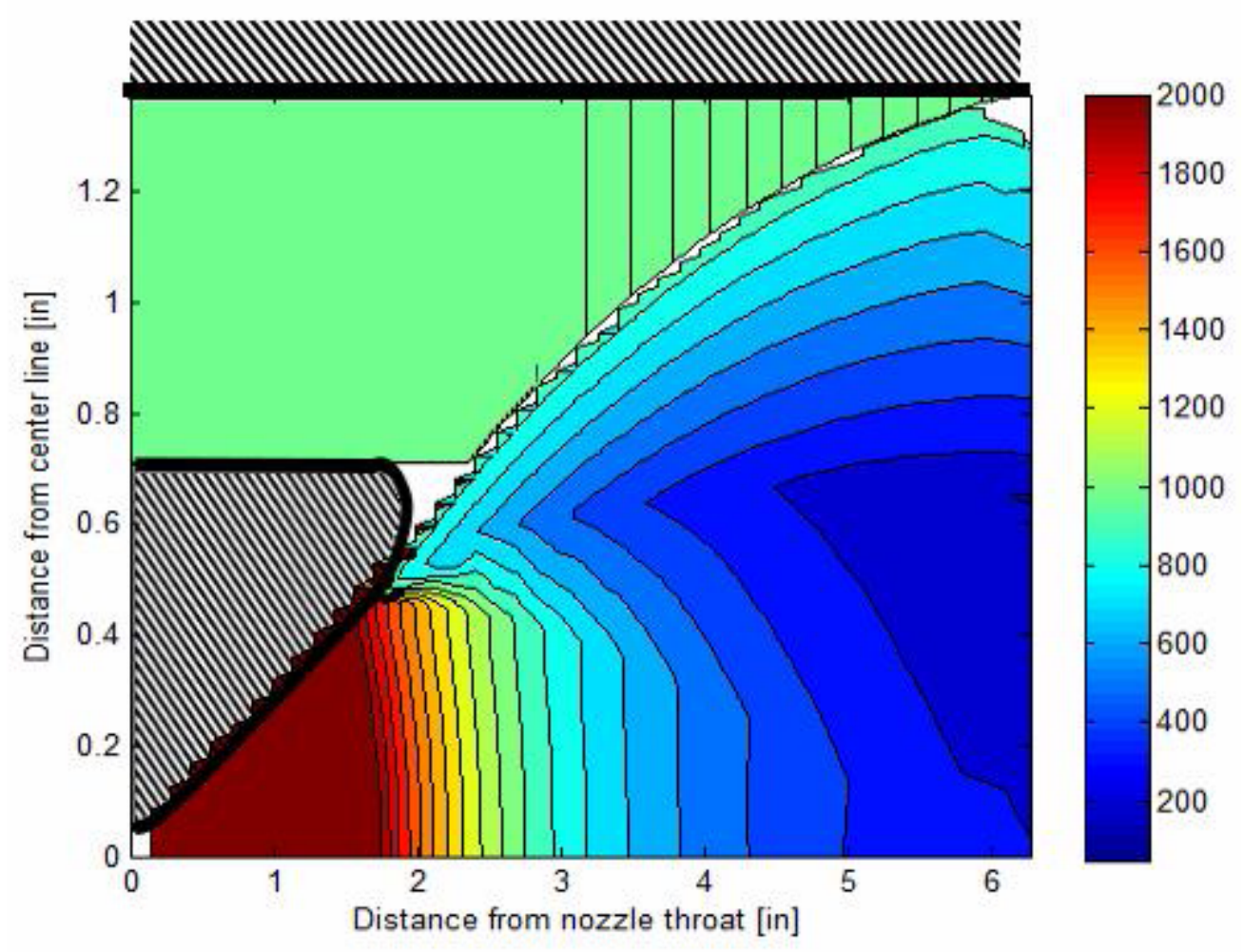

Figure 13: CPSE, PR = 250, Static Pressure Distribution of the blocked flow pattern ${ }^{11}$

Morham predicted the blocked case would occur past 4 inches from the nozzle exit plane at pressure ratios above 230. Operating as a supersonic ejector with a fixed secondary volume allows a physical reduction in mass flow rate of the secondary which become dependent on the conditions of the primary flow. 


\section{Experimental Apparatus}

The experimental apparatus contains the same basic components as previous research. Several upgrades were made to the hardware and software as well as some fundamental changes to the operation of the rocket. The system was essentially completely rebuilt, using as many previously fabricated parts as possible, although the modifications for the current set of research required a large amount of parts to be fabricated and replace parts of the apparatus.

\subsection{CPAAR Hardware}

The complete experimental apparatus can be broken up into 4 distinct components. The first is the Air Augmented Rocket. This component contains the secondary ducts, thruster and mixing duct of the rocket, which is used to produce the Fabri cases. The second component is the Plenum. This component serves as a constant volume inlet area, which allows the stagnation pressure of the secondary flow to reduce and increase the achievable pressure ratio. The third component is the Flow Control System. This component controls the flow of nitrogen to the primary thruster. The final component is the Data Acquisition System used to measure and record the experimental data during the tests. Figure 14 presents the four components in a simplified (not to scale) diagram. 


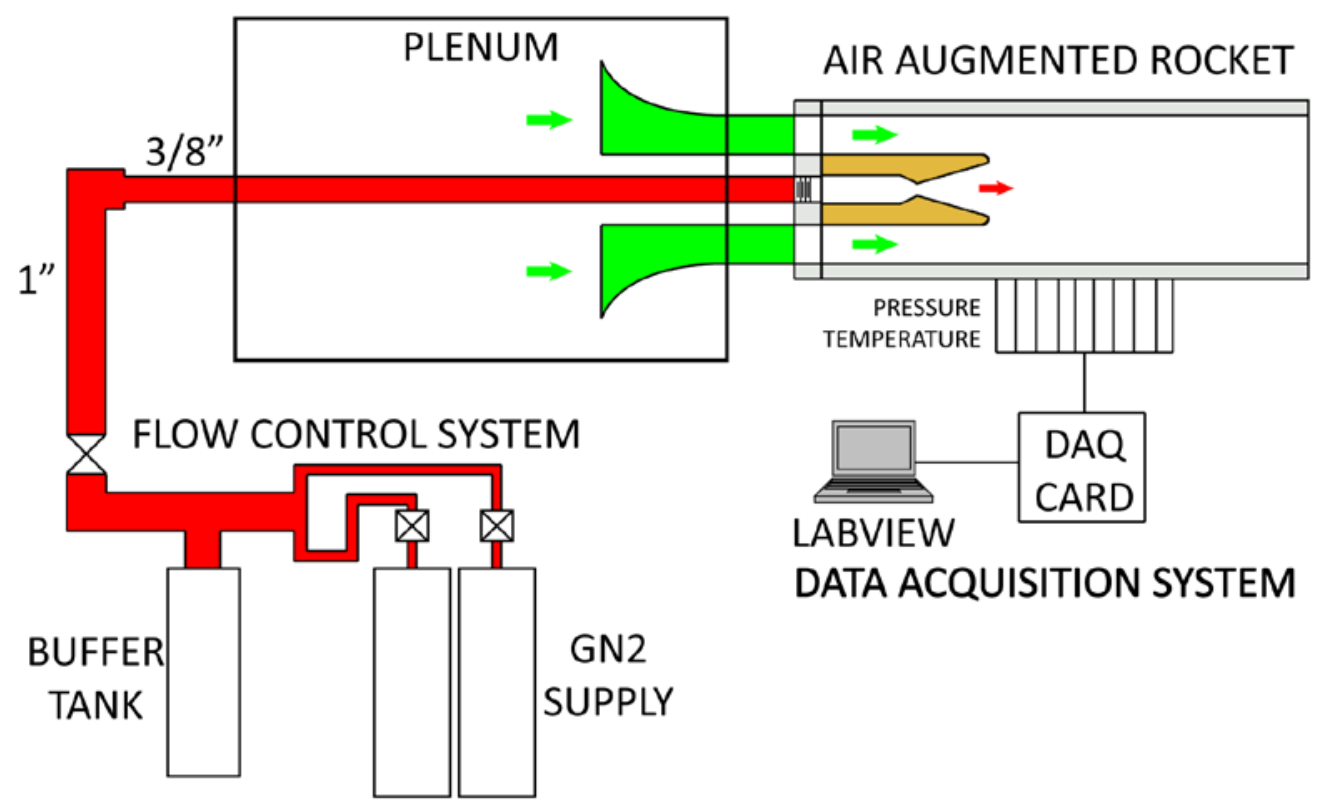

Figure 14: CPAAR Test Apparatus (Not to Scale)

\subsubsection{Air Augmented Rocket}

The Air Augmented Rocket was built and tested by former graduate students Trevor Foster and Ryan Gist for Hot flow (Methane/Oxygen Combustion) and Cold Flow (Nitrogen) Testing. The rocket was designed to approximate a 2-D thruster from the proposed ISTAR X-43B RBCC engine ${ }^{13}$. For the purposes of our experimental study the rocket was used in a cold flow configuration.

The thruster was designed and constructed originally by Trevor Foster and Ryan Gist in their study of the $\mathrm{AAR}^{4,9,10}$. The 2-D configuration of the thruster simplifies the disassembly and reassembly of the system in between tests. The thruster consists of an inlet manifold, primary thruster nozzle section, the secondary ducts, and mixing duct.

The thruster assembly is composed of the intake manifold, primary thruster and mixing duct. The inlet manifold is machined stainless steel in 3 parts, the center part is composed of a centrally located oxidizer port and two fuel inlet ports located on the top and bottom, 
these flow directly into the primary thruster combustion chamber. The two outer parts compress onto the center part and have a secondary flow duct. The manifold is bolted to the thruster bottom plate and mixing duct walls. Figure 15 is a model of the thruster assembly in previous hot-fire condition.

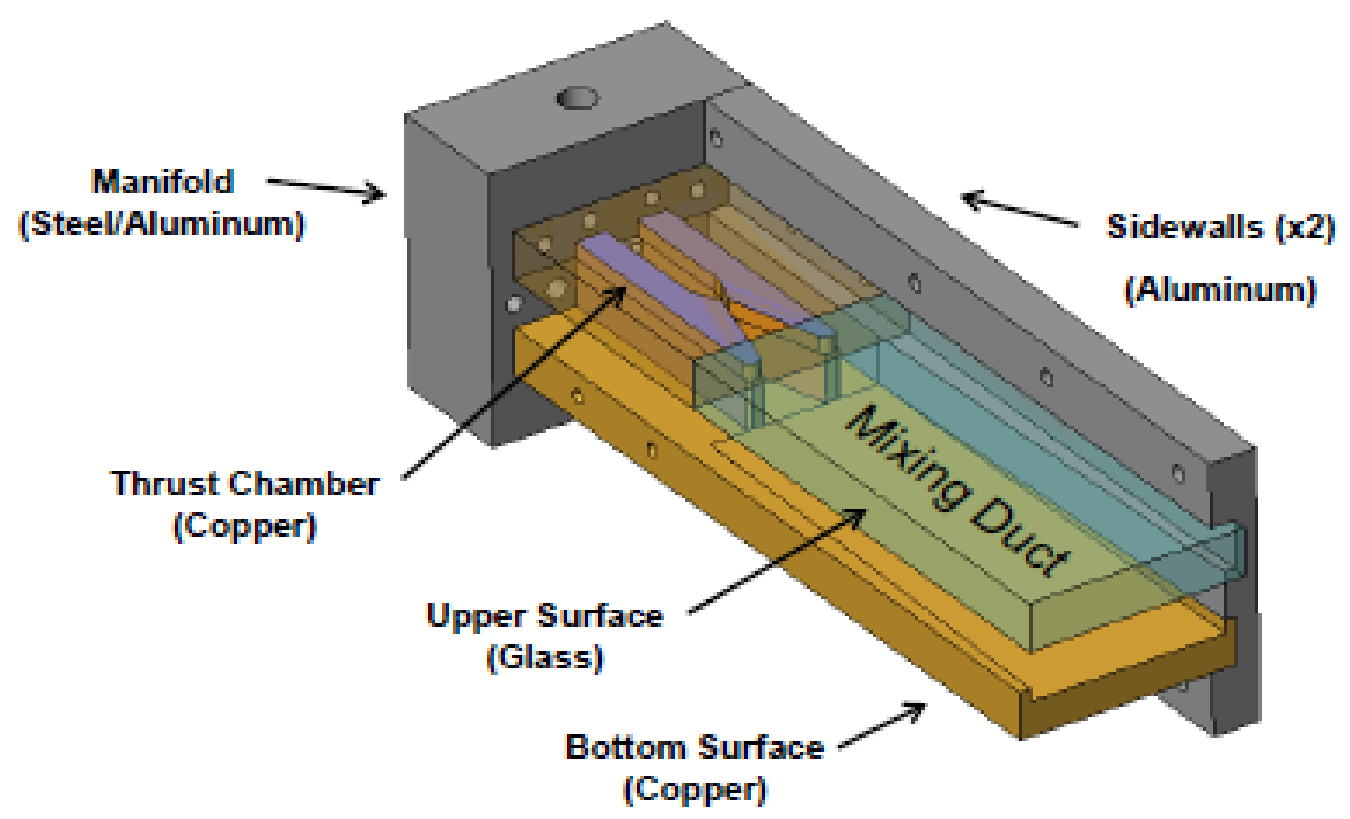

Figure 15: Model of the Cal Poly Supersonic Ejector ${ }^{10}$

The original thruster assembly was designed in reference to high heat load constraints. The area ratio of the nozzle that was tested during the current experimental investigation was 22.5. The area ratio of the nozzle in the present test series was chosen based on increasing the overall length of a test based on limited nitrogen supply capabilities.

The inlet manifold is the section that connects the feed lines for the primary flow and inlet lines for the secondary flow. The flow of oxidizer and fuel are connected in separate areas of the inlet manifold and flow into the primary combustion chamber during a hot test. During the test series, the fuel inlet ports were blocked off, and the oxidizer port 
(3/8” NPT) was where nitrogen from the buffer tank was fed to; this had no affect on the cold flow performance of the thruster.

Previous tests were conducted in the configuration of the Figure 15, with a glass upper surface to observe the flow in the mixing duct. The duct walls diverge at 1.5 degrees to prevent the presence of a normal shock and encourage an oblique shock train ${ }^{9,14}$. The bottom copper surface was machined as a one piece with the chamber and mixing duct surfaces. Pressure transducers were placed along the centerline of the mixing duct to record properties of the primary flow. The secondary flow of the rocket entrained air from ambient atmospheric conditions, and limited the pressure ratios that could be achieved.

The mixing duct walls on the rocket were lengthened to rectify problems of exit pressure influence on the expansion of the primary plume in the mixing duct as previously observed by Gist ${ }^{10}$. This corresponds to the work of Addy, who suggests that a width to diameter ratio of 9 is optimal for an air-to-air supersonic ejector ${ }^{7}$. Addy's work is based on axis-symmetric flow, but based on the hydraulic diameter of the mixing duct, the original design of the mixing duct was less than half as long as what would be suggested by Addy. The mixing duct length was increased from 8 to 18 inches as shown in Figure 16. 


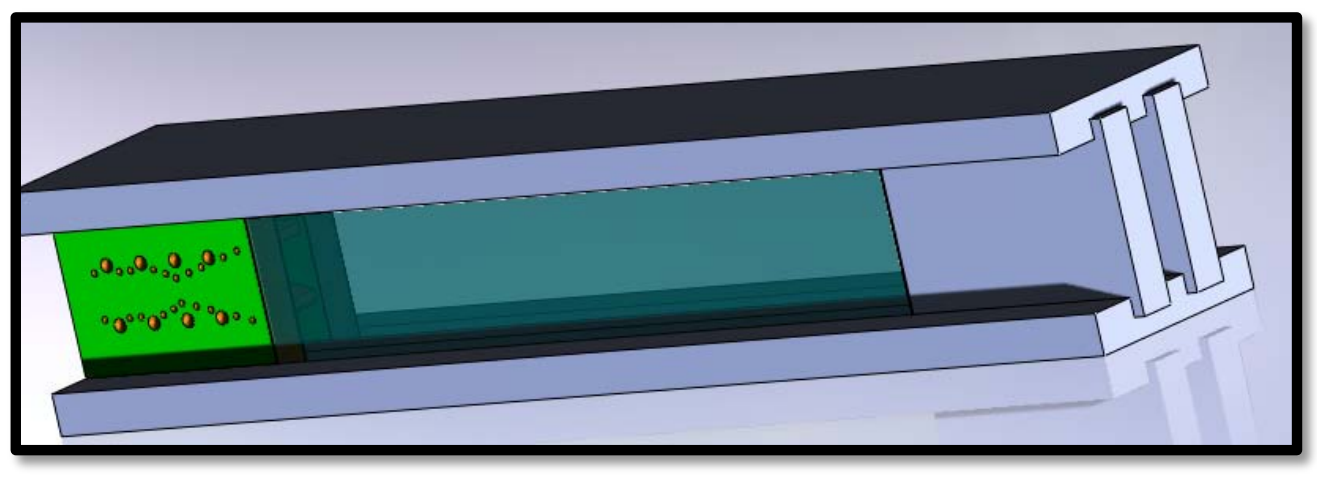

Figure 16: Mixing Duct Extension Model

As the mixing duct length was increased, the original bottom and top glass plates of the mixing duct were no longer able to be used. For current series of testing, flow visualization techniques would be incorporated for verification of flow conditions in the mixing duct. To visualize the flow in the mixing duct to a higher fidelity than previous research, a Schlieren imaging system was developed.

When the Schlieren image system was not in use, a solid aluminum plate was placed on the bottom of the assembly and instrumented with pressure transducers along the centerline of the primary flow to observe the shock structure of the primary flow.

\subsubsection{Secondary Duct Vacuum Plenum}

To achieve the higher pressure ratios required for a Fabri-choke and Fabri-block case, Gist and Foster suggested the addition of a low pressure plenum attached to the inlet of the secondary ducts. Such a plenum would allow the stagnation pressure of the secondary duct to be reduced to a point where the resulting high pressure ratios could effectively simulate a high-altitude launch condition.

The design of the Schlieren imaging system as well as the design of the rocket apparatus, created significant restrictions on the design of the plenum that could be developed. Due 
to these constraints, a system was designed that utilized the same principles but was not identical to the secondary flow system developed by Addy.

Several ideas for a plenum were considered, including apparatus' that encompassed the thruster of the AAR configuration as suggested by Foster and a system that connected to the manifold via intake tubing. It was determined in order to use the Schlieren imaging system available it would not be possible to develop a cost effective system capable of encompassing the entire thruster. The system chosen would use ducts or tubing to connect the secondary ducts of the inlet manifold to the plenum.

The plenum had several requirements once a configuration was chosen, the requirements included manufacturability, ease of disassembly, low cost and containing a large volume. The volume of the plenum dictates the rate of pressure change in the plenum for a given primary thruster and inlet configuration. The objective was to achieve a short startup transient time compared to the total test time ${ }^{7}$. According to Addy, the percentage of overall test time correlates the quasi steady region to operation at steady state. To achieve this quasi-steady transient operation in relation to the mass flow rates the primary thruster is capable of, it was calculated that the volume of a standard 55-gallon drum was roughly similar to the size requirement. The calculation was based on the mass flow rate of the secondary during supersonic saturated operation simulated by the CPSE simulation code.

A standard 16-gauge oil drum with steel reinforced skeletal structure was found to be able to handle the loads required. An analysis on the loads the oil drum would experience was done, based on the ambient pressures it was designed for. The internal design pressure of the plenum was chosen to be around 2.5 psia based on initial calculations using saturated mass flow rates (worst case scenario) retrieved from the CPSE 
Simulation. The design pressure Based on the ASME code for pressure vessels ${ }^{15,16}$, the thickness required for short cylinders with external pressure is:

$$
\frac{t h}{O D}=\left[P\left(\frac{1-v^{2}}{2 E}\right)\right]^{\frac{1}{3}}
$$

The thickness of the oil drum was too small for the desired pressures in the plenum to achieve high pressure ratios. To strengthen a thin walled pressure vessel such as the oil drum, an internal structure capable of supporting the load induced by pressure being applied externally was required. The resulting structure was composed of a structure of square steel tubes. A tube was placed along the length of the drum and connected to a tube on the opposite side by two 5/8” threaded steel rods. Threaded rods were used to compress the tubes along the entire wall of the drum. In total, 8 rods were used to support the walls of the drum, as shown in Figure 17. 


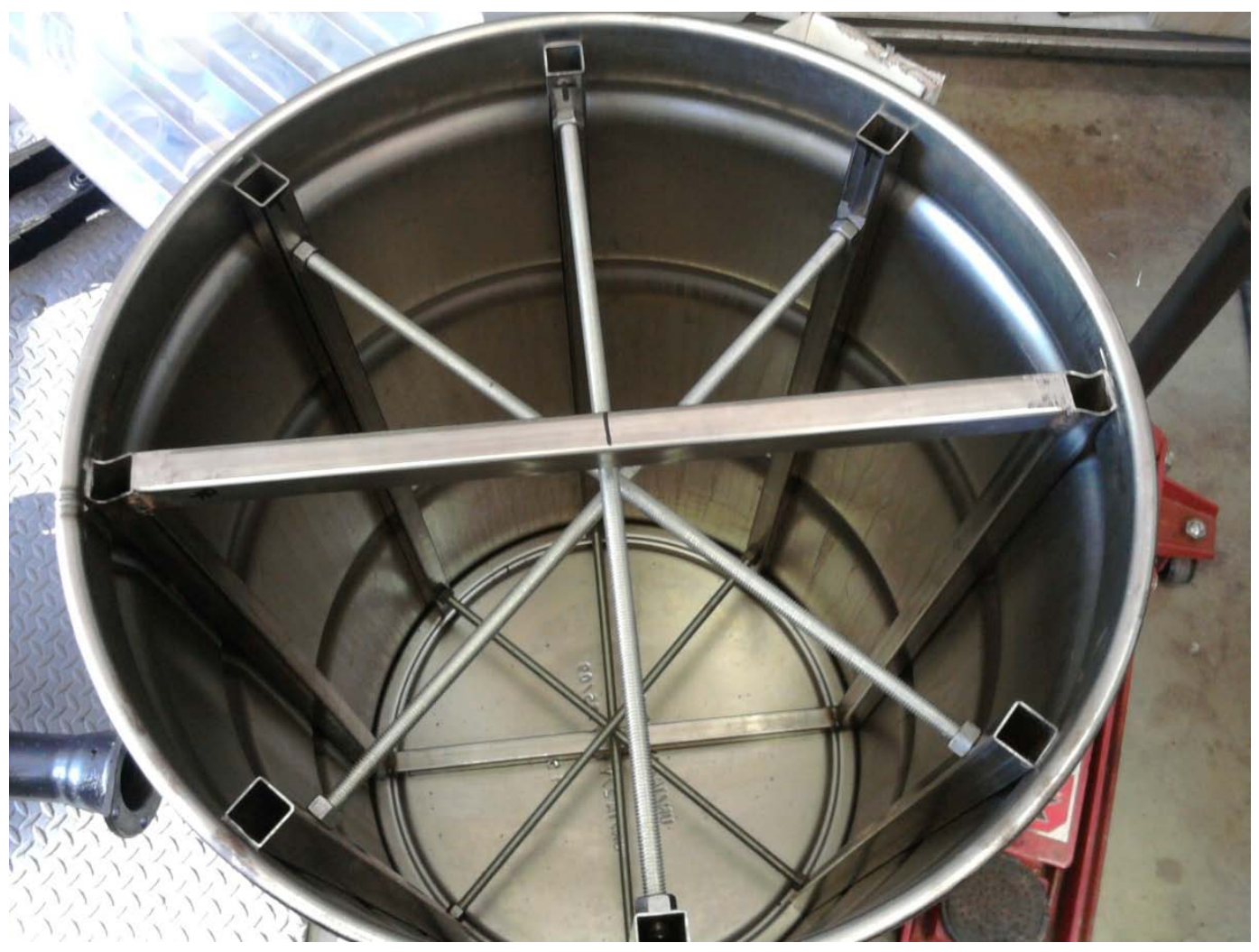

Figure 17: Plenum Support Structure

For simple pressure vessels, a factor of safety of 3 to 4 is considered acceptable ${ }^{15,16}$. With this set up the factor of safety achieved is dependent on the minimum pressure of the drum. To obtain an acceptable factor of safety and retain a quasi-steady assumption the internal pressure of the plenum was assumed to be 0.7 psia, a 14 psi pressure differential from standard atmosphere. To determine if the load experienced is greater than what the system can handle, the loads experienced by the column must be calculated. Typically with a column experiencing compressive force in the axial direction, the critical buckling load of the configuration will cause the failure. The equation for buckling on a column is presented below. The load was calculated with a simple static distributed load along the length of the wall support beam. 


$$
F=\frac{\pi^{2} E I}{(k L)^{2}}
$$

The factor $\mathrm{k}$ is equal to 0.5 for a column fixed at both ends. The factor of safety of the system is calculated by dividing the critical load required for buckling the support by the loads experienced by the supports. The resulting factor of safety of the system is 3.8

Due to clearance issues with the primary flow piping, the inlet piping had to be slightly flexible in order to bend around some of the high pressure connections of the primary flow piping as shown in Figure 18. The initial design of the inlet system introduced several different challenges in the characterization of the secondary flow during tests. With the first and second iteration of the secondary flow piping, there was observable flow disturbance similar to the issues with flow recirculation and choking in the secondary duct upstream of the mixing duct. These are the same issues encountered during the high pressure cold flow testing done by Gist ${ }^{4}$. The original assumption was to obtain pressure ratios high enough that the area of the choke point of the secondary flow would at a certain pressure ratio, be smaller than the minimum area of the secondary duct geometry thus allowing for the choke point to be in the mixing duct. The upstream secondary flow choking was observed in the data in early tests, and it was determined the flow could no longer be assumed as isentropic during this type of operation. 


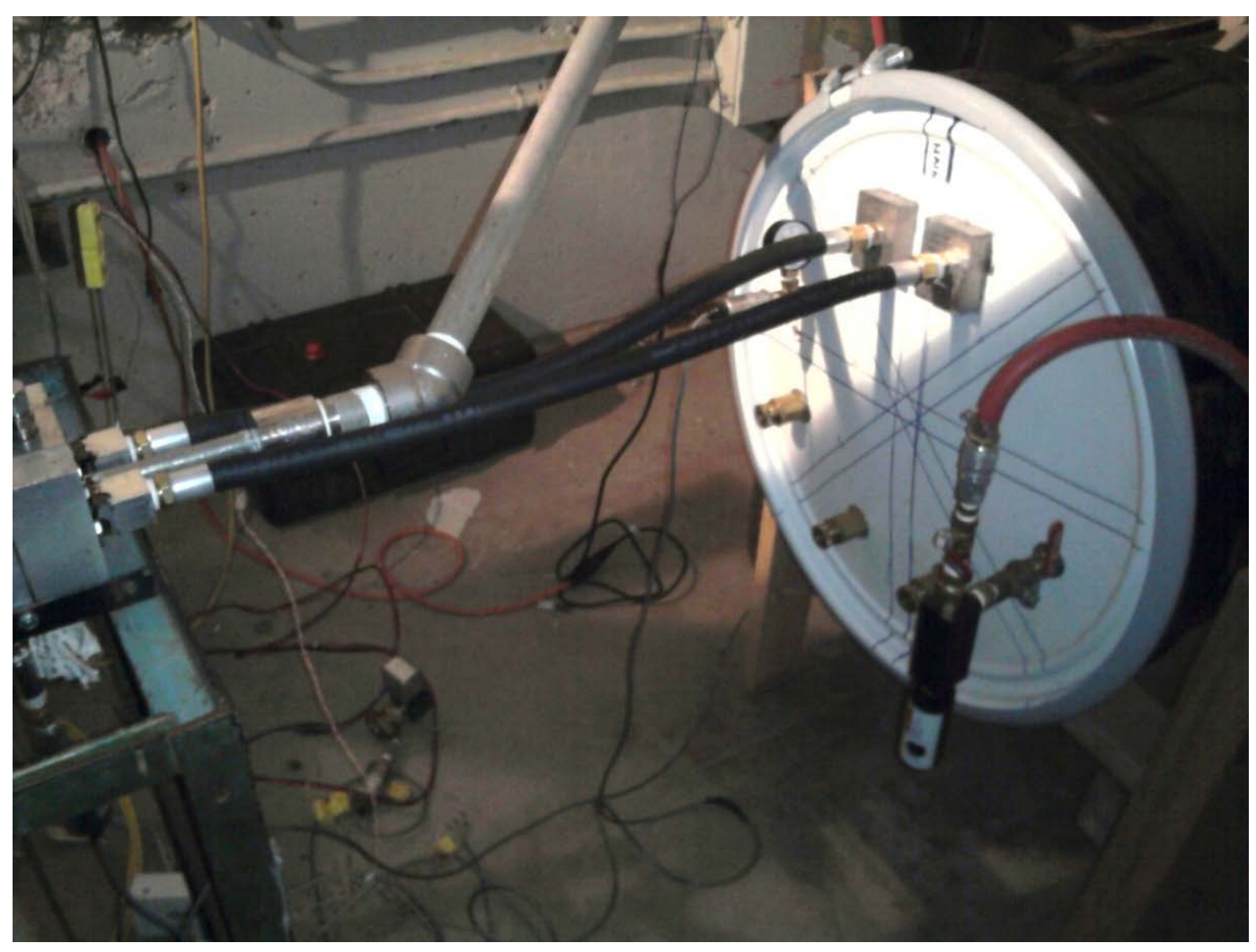

Figure 18: Feed System, Iteration 1

Several methods were employed to reduce the stagnation pressure of the secondary flow. First, the plenum pressure could be reduced to a certain pressure before the test from a vacuum pump attached to the plenum and then reduced further from entrainment due to the primary flow. Another method had the plenum at ambient pressure before the start of the test and only used the primary flow entrainment as the method of reducing the pressure of the plenum. The first method was attempted in the first two iterations of the intake system, although this produced some interesting challenges. To reduce the pressure of the plenum before the test, the plenum must be isolated from the ambient atmosphere; two methods of this were developed: using a burst disc to isolate the plenum from the secondary ducts, and using a block at the end of the mixing duct to isolate the plenum and rocket from the ambient atmosphere. 


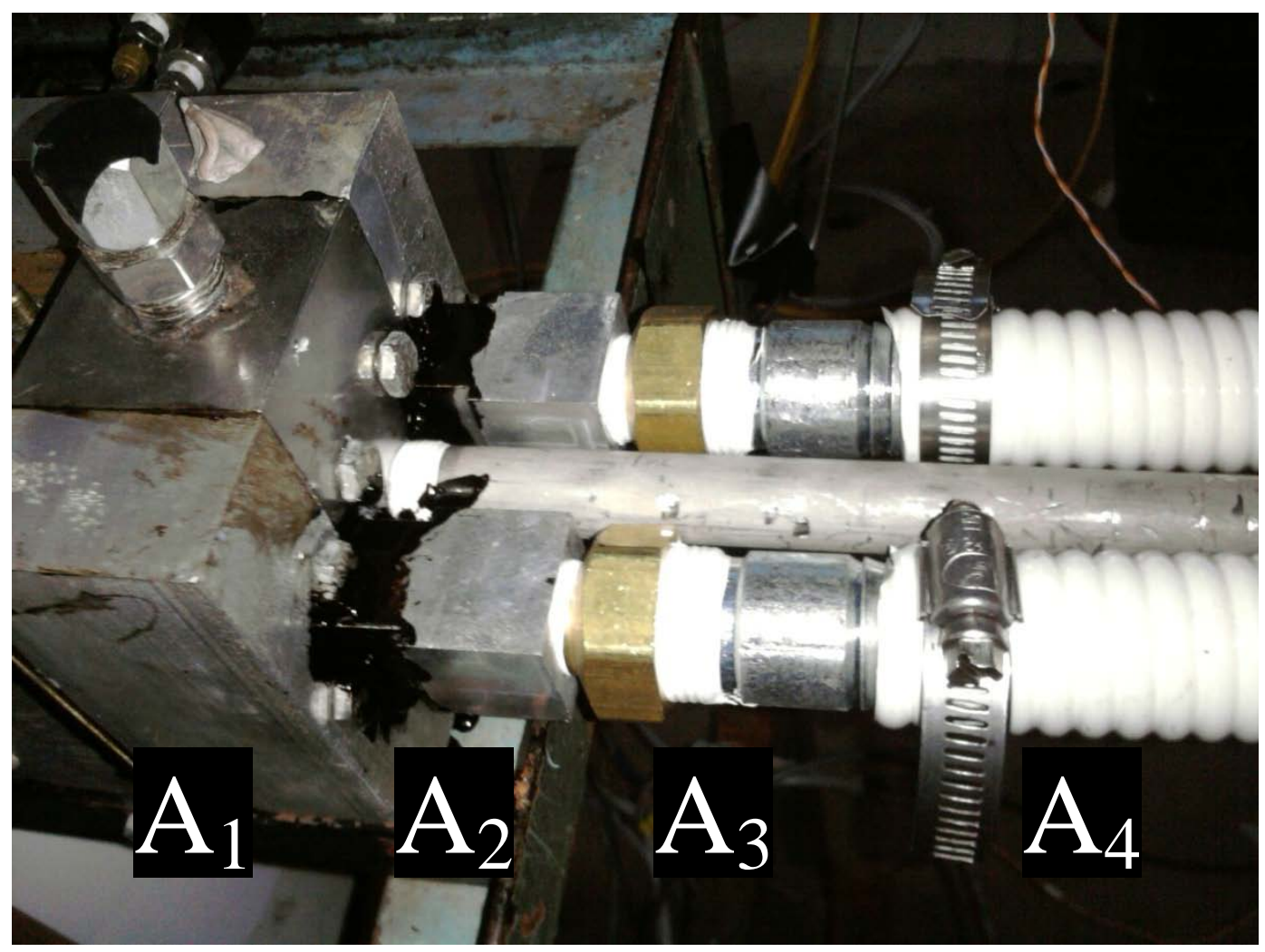

Figure 19: Rocket Manifold Feed System Inlet, Iteration 2

The ducts were not able to have a straight connection from the rocket manifold to the plenum; the flow could no longer be assumed a straight flow. A circular to square transition was introduced to the secondary ducting as a result of a flexible and changing area inlet system. An adapter for this transition was machined out of aluminum, which connected the secondary duct of the rocket to the piping via a $1 / 2$ " NPT connection. Initially the flex line was a $1 / 2$ ” NPT line; this introduced very extreme area changes into the flow at the tubing connections ( $A_{4}$ to $A_{3}$ in Figure 19). These abrupt area changes introduced turbulent flow and recirculation regions and invalidated the isentropic flow assumption used to analyze the recorded data, as well as introducing a choke point in the system. In the second iteration of the inlet, the flex line was increased to a 1" NPT line, which shifted the minimum area to be at the circular to square transition just before the 
secondary duct ( $A_{1}$ to $A_{2}$ in Figure 19and Figure 20). The relative areas of different components of the secondary inlet system are represented in Figure 20.

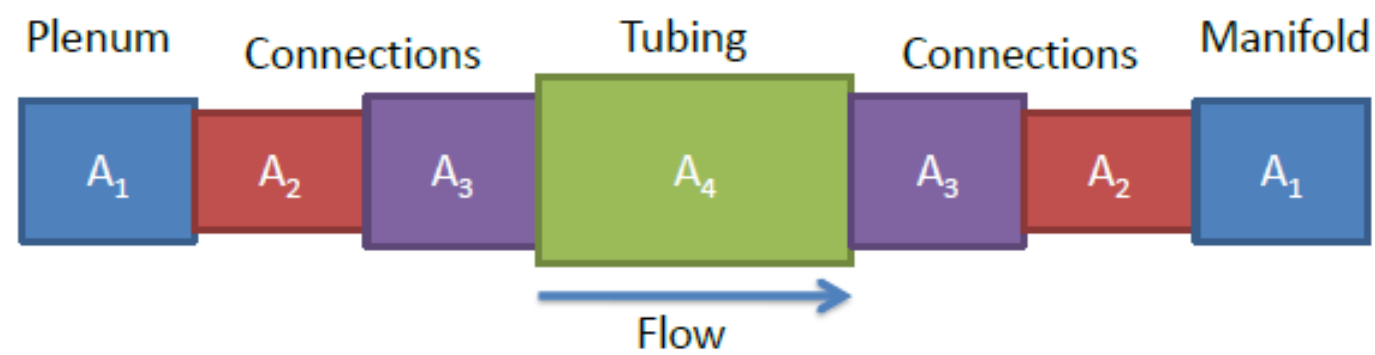

Figure 20: Secondary Inlet Area Differences Diagram, Iteration 2

The minimum area of the system was at the connections, upstream of the manifold, Figure 21. The minimum area created a choke point upstream of the secondary duct of the rocket. The saturated case would occur with a smaller choking area, resulting in a lower mass flow rate. The pressure ratio required to produce saturated case would be higher than if the minimum area of the secondary flow was larger. 


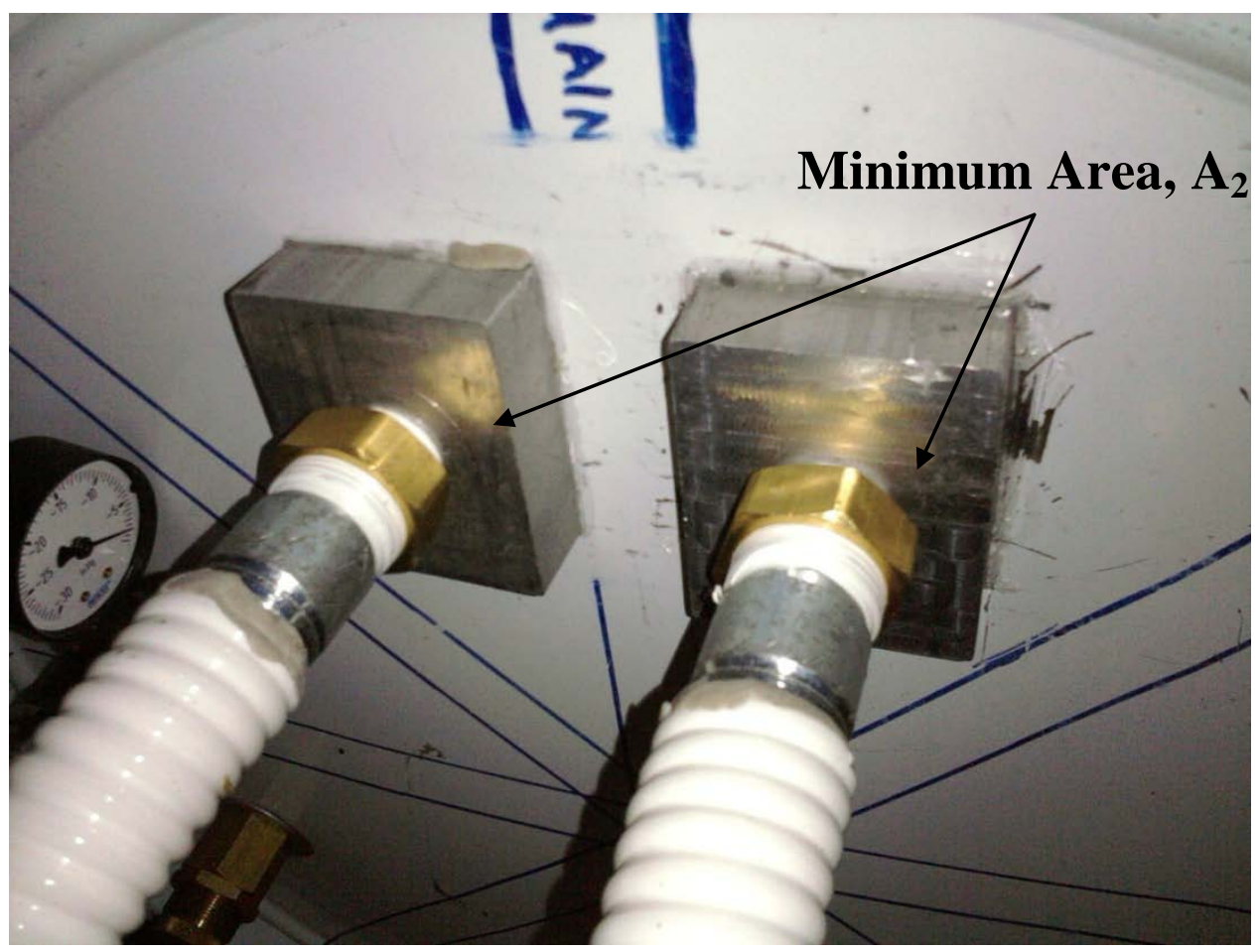

Figure 21: Secondary Duct, Plenum to Inlet Connection

Without a constant area duct for the secondary duct, the flow cannot be assumed to be isentropic due to losses in the inlet. The flow had choke points upstream of the secondary duct due to the minimum area being in the inlet piping, which increases the pressure ratio required for the saturated secondary flow case.

To solve the problem of choking upstream of the secondary ducts, a completely new system of flow intake to the manifold was devised. The secondary ducts would be attached to constant area inlets, which have the same cross-section as the secondary duct. The inlets would be attached directly to the plenum through an interface plate. The interface plate would allow an extended primary flow tube to pass directly through the plenum to the manifold and the secondary inlets to be exposed to the plenum. A crosssection model of the system is presented in Figure 22 and Figure 23 and shows how the 
system allows for the primary and secondary flows to remain isolated from each other but also remain strait.

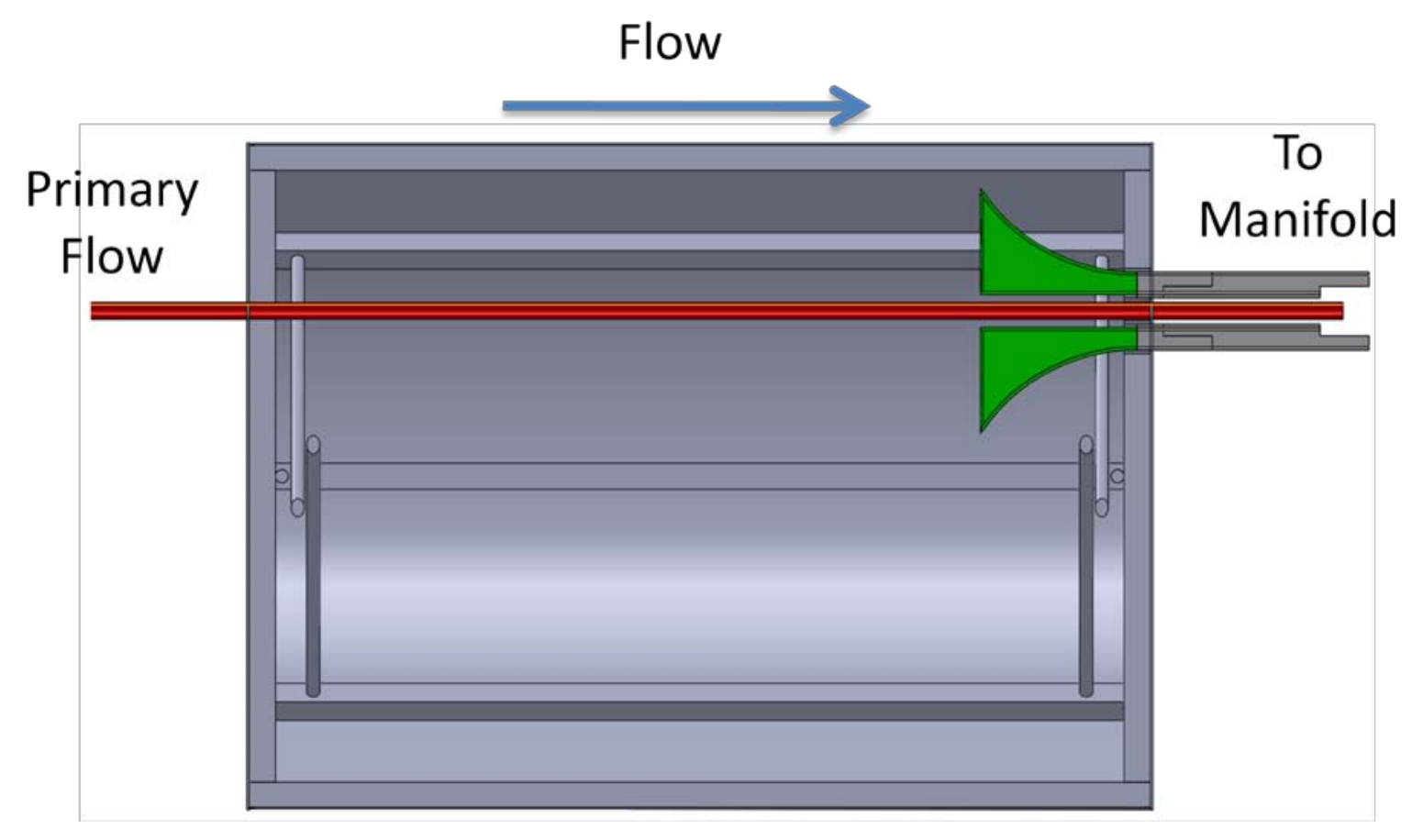

Figure 22: Flow Path for Inlet Configuration Iteration 3

The third iteration of the intake system for the secondary ducts was designed to remove all possibilities of upstream choking on the secondary ducts while minimizing losses in the system. 


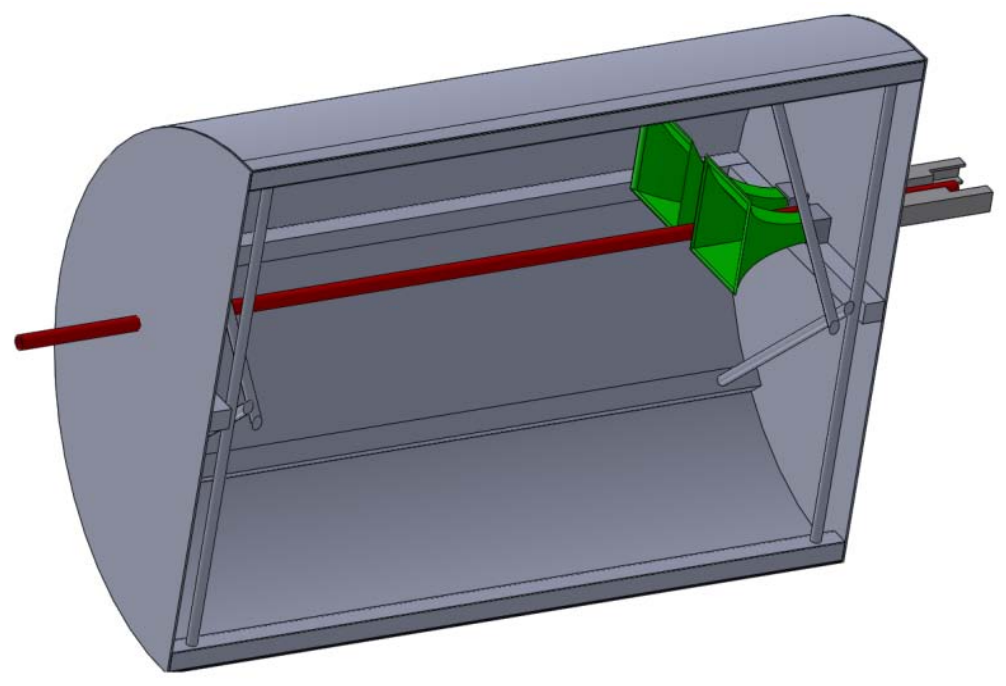

Figure 23: Model of Plenum for Inlet Configuration 3

The pipe colored red is the 3/8" Stainless steel pipe for the primary flow. The outer diameter of the primary flow pipe interfaces directly with the interface plate at the walls of the plenum which are supported by the steel structure. The primary issue becomes sealing of the interface of the pipe and the wall of the plenum. The primary flow pipe was a single stainless steel pipe that went through holes on both ends of the plenum with a hole-fit tolerance of $+/-0.01$ ”. This method was chosen as opposed to using union connections at the walls, because it keeps the flow area uniform and does not introduce discontinuities along the flow walls. The decision to use a one piece pipe was made based on reducing the amount of fittings in the high pressure primary line. Using two separate interfaces on the plenum would increase the probability of leaks in the high pressure line and could reduce the performance of the CPAAR as well as increase the risk of damage to the apparatus and equipment. The increase in length of the line was determined not to produce any significant line losses. The remaining space is covered with vacuum rated RTV and vacuum putty. 
The secondary inlet system was made using same inlet horns used in previous testing ${ }^{4,9}$. The inlet horns are ABS plastic and were rapid prototyped, they were designed to facilitate laminar flow in the secondary ducts. The secondary ducts also interfaced at the wall of the plenum were sized such that the distance between the two ducts was sufficient for clearance of the primary flow pipe. The lengths of the ducts were sized such that the plenum would have sufficient clearance from the CPAAR for maintenance and troubleshooting issues, as shown in Figure 24.

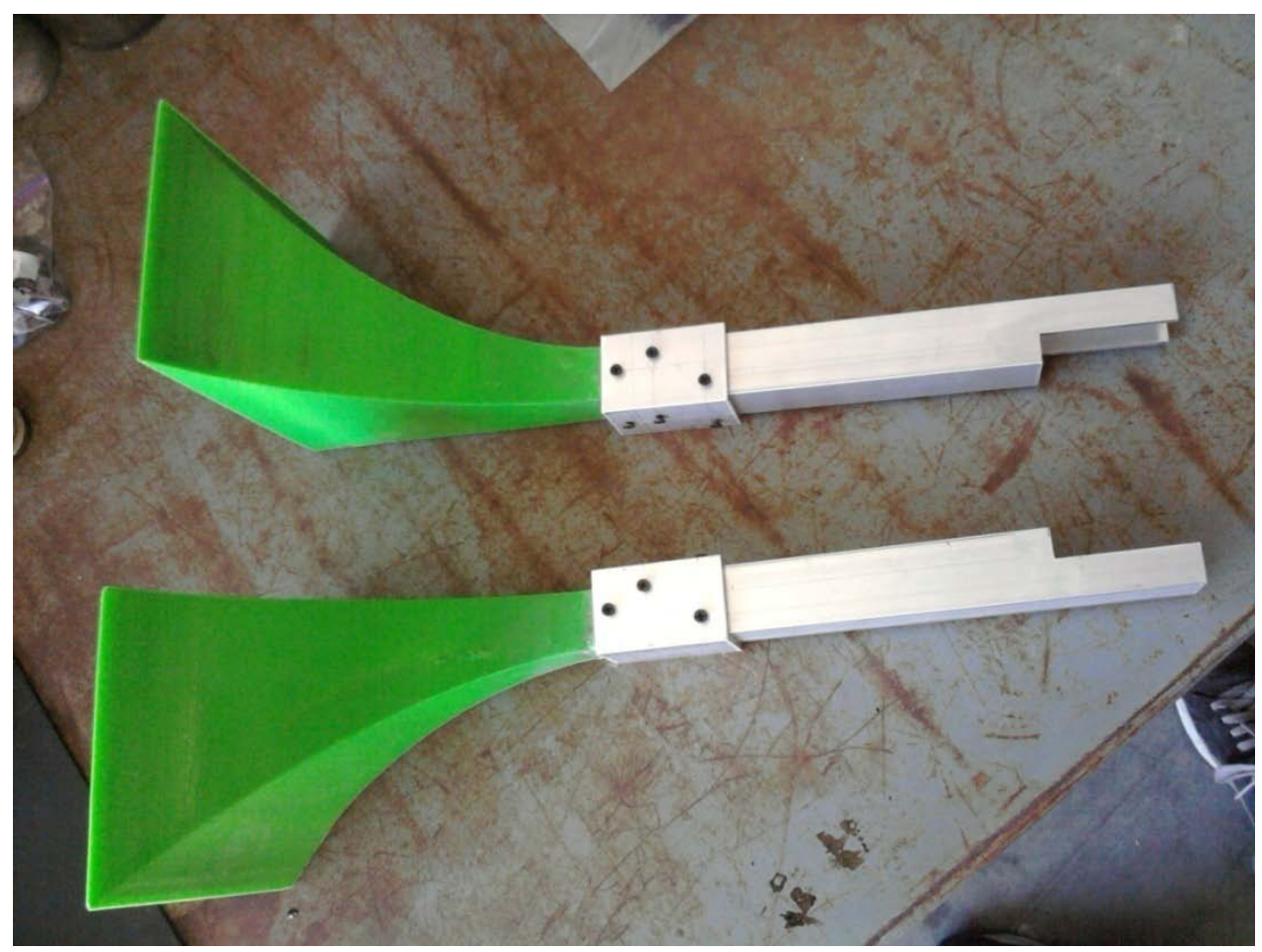

Figure 24: Secondary Duct Configuration, Inlet Horn and Inlet Extension

In the time between Fosters testing and the initial build up of this testing, during storage of the inlet horns, the part of the inlet which fits into the manifold had broken off completely such that they could not retain their position in the inlet manifold. Adaptors connecting the inlet horns to the manifold of the rocket were machined out of 6061 aluminum and kept the secondary duct a constant area. The addition of these adaptors 
increased the length of the secondary duct by 7 inches, the length of the adaptors were determined by the minimum space required to assemble the new intake system.

The adaptor and inlet horn were sealed together by epoxy adhesive, essentially becoming a long inlet. The new adaptor system can be used independent of the plenum as well, to potentially be applied to non-transient experiments of the CPAAR.

The losses in the new inlet system can be seen by the change in stagnation pressure from the plenum pressure to the secondary duct, shown in section 7.2.1. The losses were not determined to be significant or detrimental to operation.

The inlets were sized such that the placement of them in the plenum would accommodate the size of the primary flow piping and interchangeability. In the case where atmospheric tests need to done, the plenum can be disassembled and inlets removed and used for further testing as shown in Figure 25. 


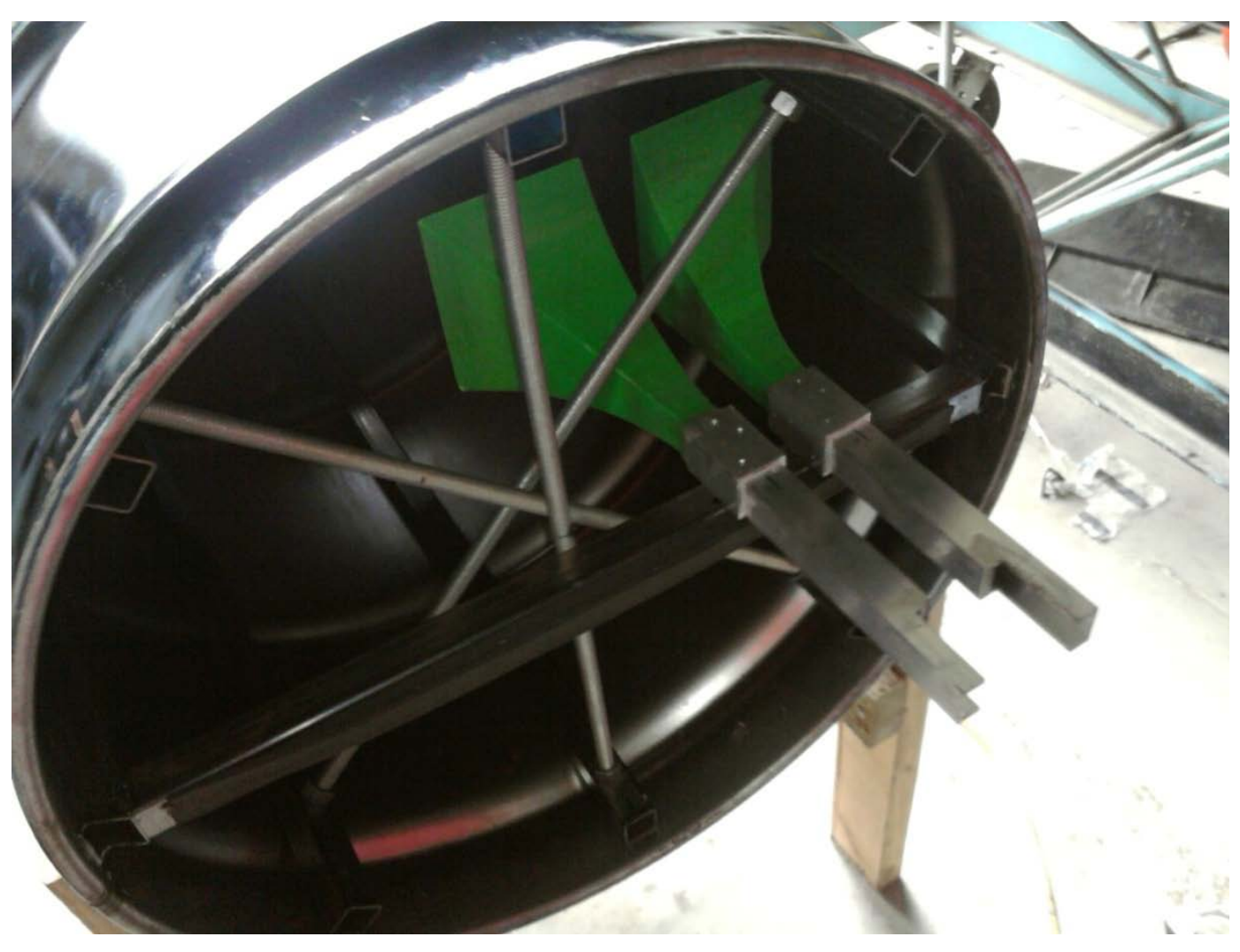

Figure 25: Secondary Duct Placement in Plenum

The inlets interface through the front of the plenum and are secured to a support beam of the plenum. An interface plate was constructed to secure and reinforce the area where the primary and secondary flows are located. The completed inlet setup is pictured in Figure 26 and Figure 27. 


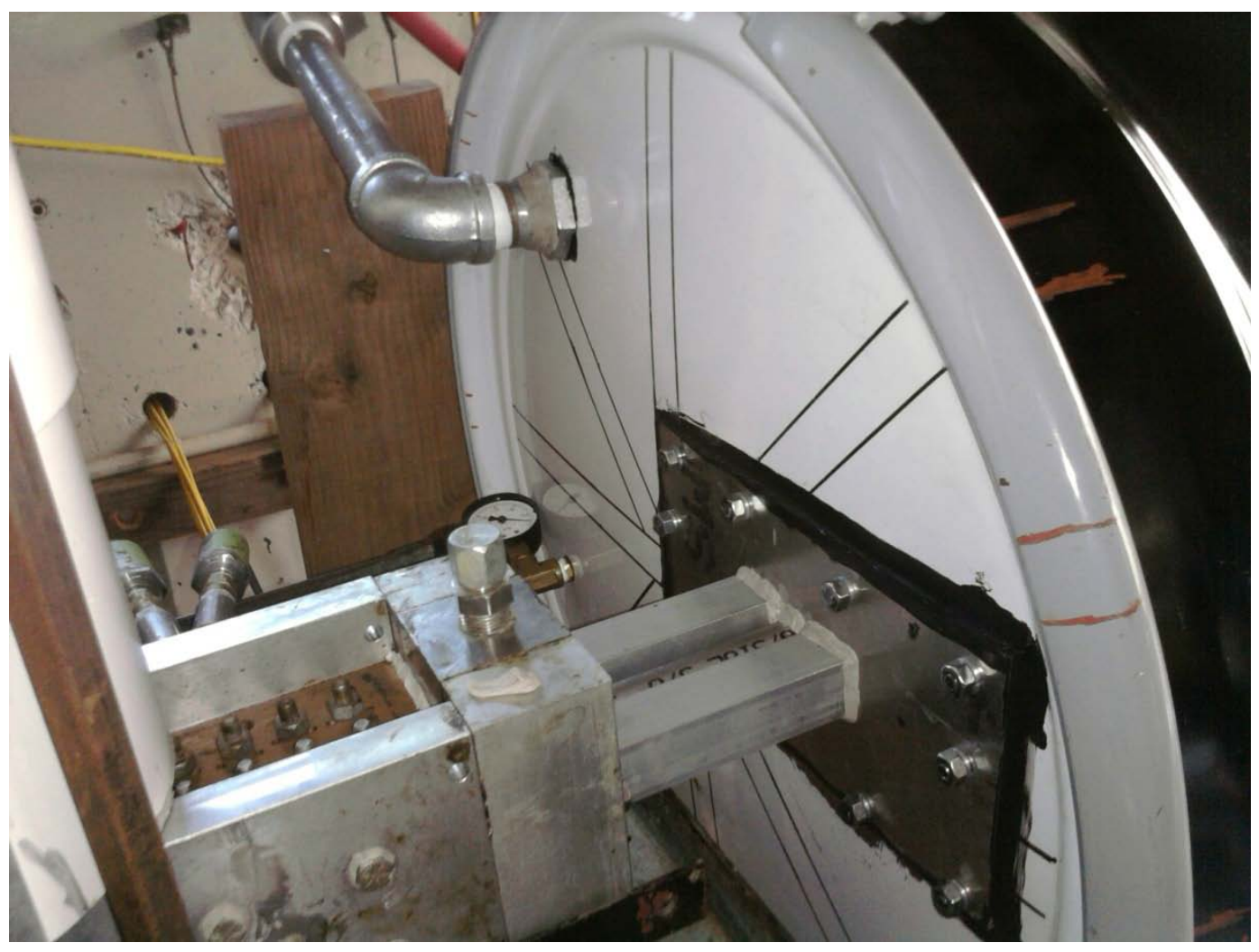

Figure 26: Support Plate with Secondary and Primary Inlets Installed, Side View

The interface plate is aluminum with machined holes for the primary flow pipe and secondary duct inlets. The plate is secured to the plenum with ten (10) 1/4" bolts. The plate is sealed with vacuum rated RTV. A hole in the plenum wall was also added for interface with the remotely actuated relief valve. 


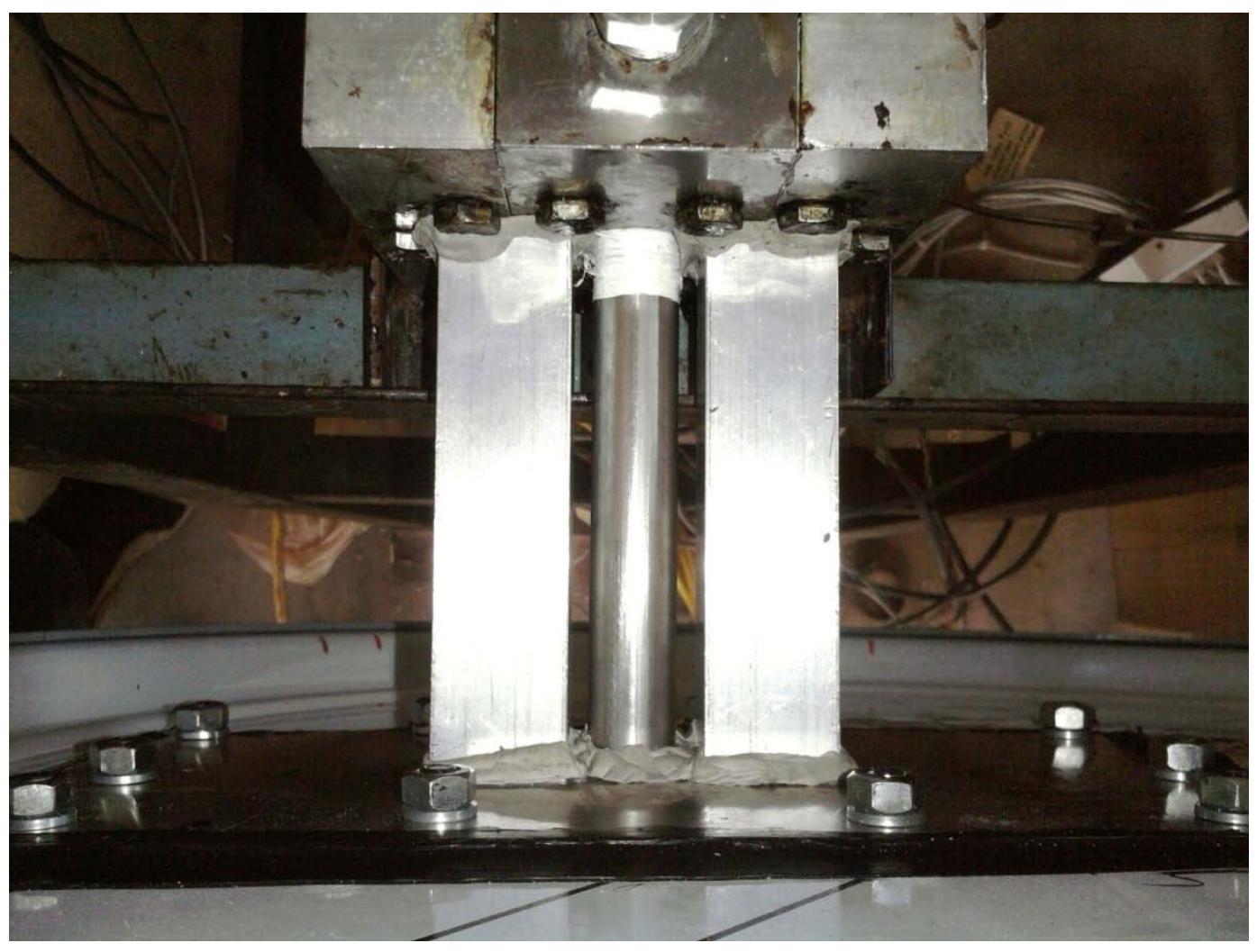

Figure 27: Interface Plate with Secondary and Primary Inlets Installed, Top View

The two secondary inlet ducts (outer position) and primary flow pipe (center) have three separate interface holes with the interface plate. Leaks are controlled with RTV and vacuum putty. The system produces flow that has less turbulent flow than previous iterations while isolating the secondary flow from atmospheric pressure.

\subsection{Flow Control System}

The Flow Control System (FCS) consists of two separate entities, the primary flow control and secondary flow control. The primary FCS's purpose was to transfer high pressure nitrogen from the storage tanks to the primary chamber of the rocket. The secondary FCS's purpose was to regulate the pressure in the plenum. 


\subsubsection{Primary Flow Control}

Two Airgas model 300 tanks containing 304 SCF of nitrogen to pressurize the buffer tank for the primary flow. The buffer tank was originally designed to facilitate pre-mixing of methane and oxygen for hot-fire tests in previous research ${ }^{9}$. The primary flow feed system components were designed to have a 1.5 factor of safety on the Airgas model 300 working pressure rating (2600 psi); the feed lines are rated to $3600 \mathrm{psi}^{9}$. The two high pressure cylinders are used in succession to pressurize a buffer tank and are pictured in Figure 28 and Figure 29.

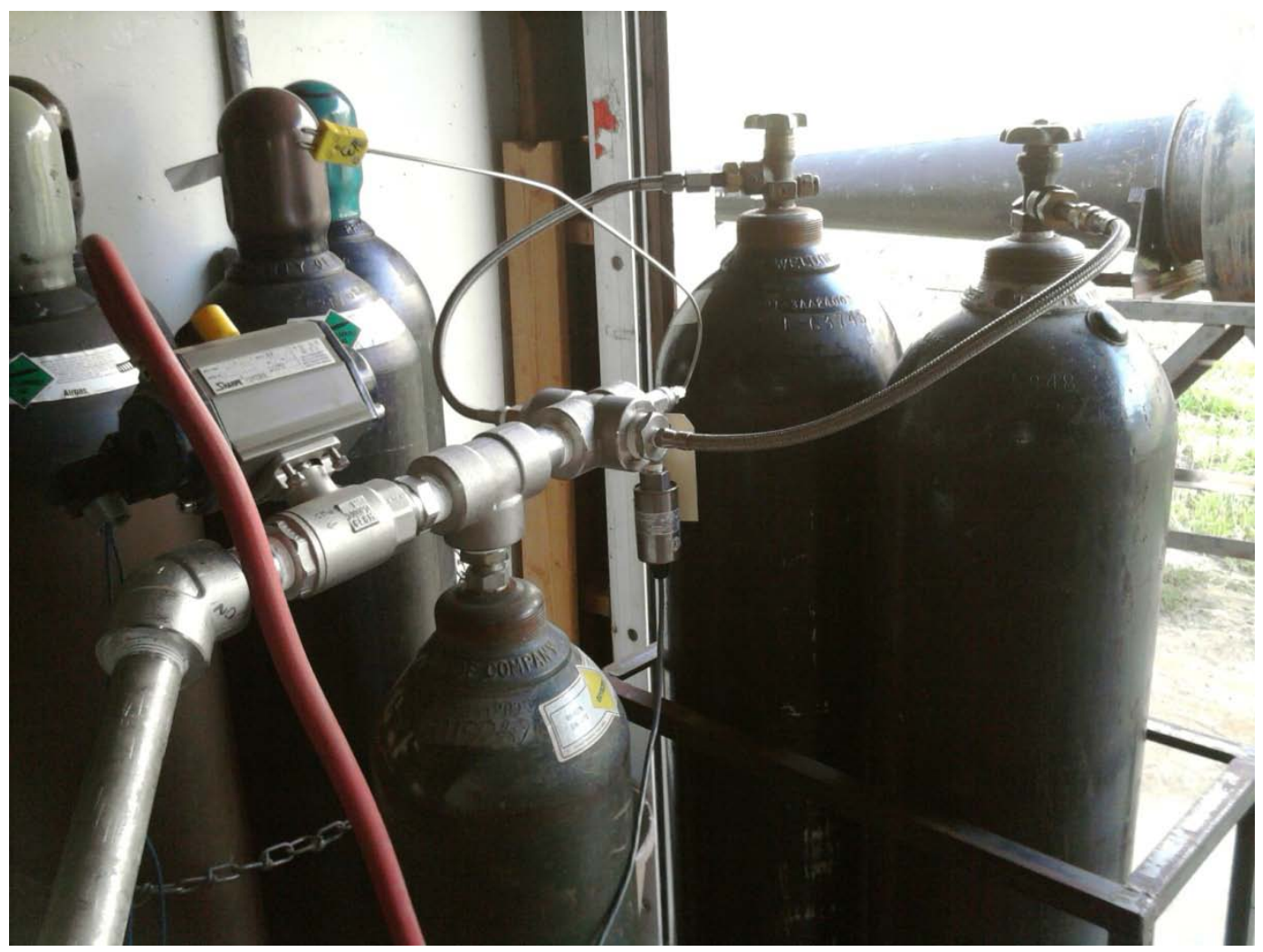

Figure 28: Primary Flow Pressurization System

The buffer tank was a standard 200 SCF gas cylinder with the original CGA valve removed and replaced with a stainless steel connection to the primary flow. The buffer tank was pressurized by connecting a single previously used nitrogen tank to the buffer 
tank and equalizing the pressure, the first nitrogen tank was then closed and a second full nitrogen tank was connected, and the two tanks were allowed to equalize again. This method pressurizes the buffer tank to the pressure of the second Nitrogen tank (up to 2200 psig). Flow was controlled by a pneumatic solenoid valve.

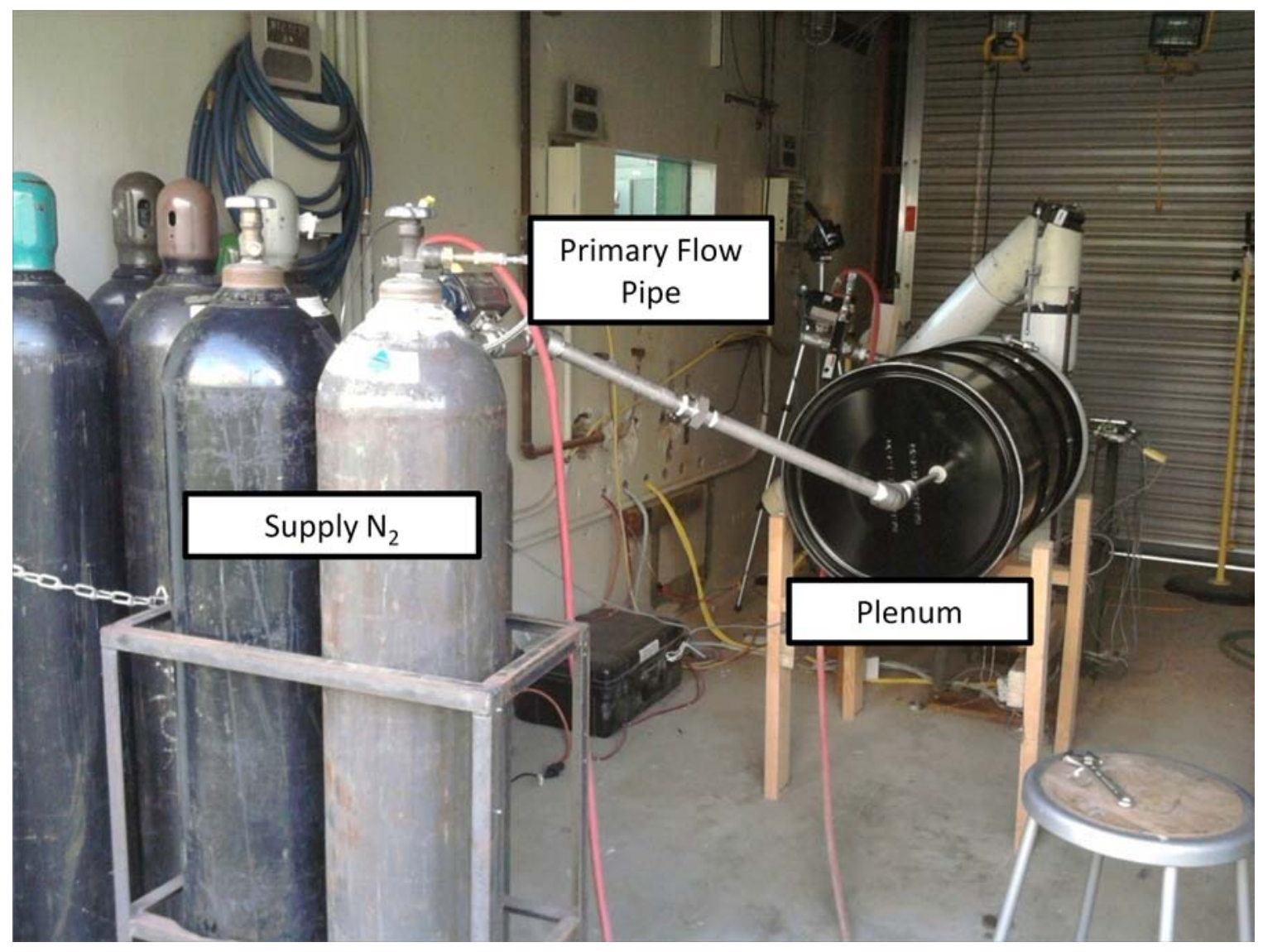

Figure 29: Primary Flow Pressurization System

A two piece Pneumatic Solenoid and 1" NPT Ball valve was coupled to regulate the actuation of the primary flow. The flow is then routed through 1" NPT piping, reduced to 3/8” NPT piping and connected to the oxidizer inlet of the intake manifold. The fuel inlets have been sealed with AN caps. All of the piping for the primary flow is 304 Stainless Steel. 


\subsubsection{Secondary Flow Control}

In the first two iterations of the secondary flow control were simple low-cost relief valves, the valves open and close relative to the pressure differential across the openings. The size of the opening is determined by the force exerted on the spring by the pressure differential between atmosphere and the pressure inside the plenum as pictured in Figure 30.

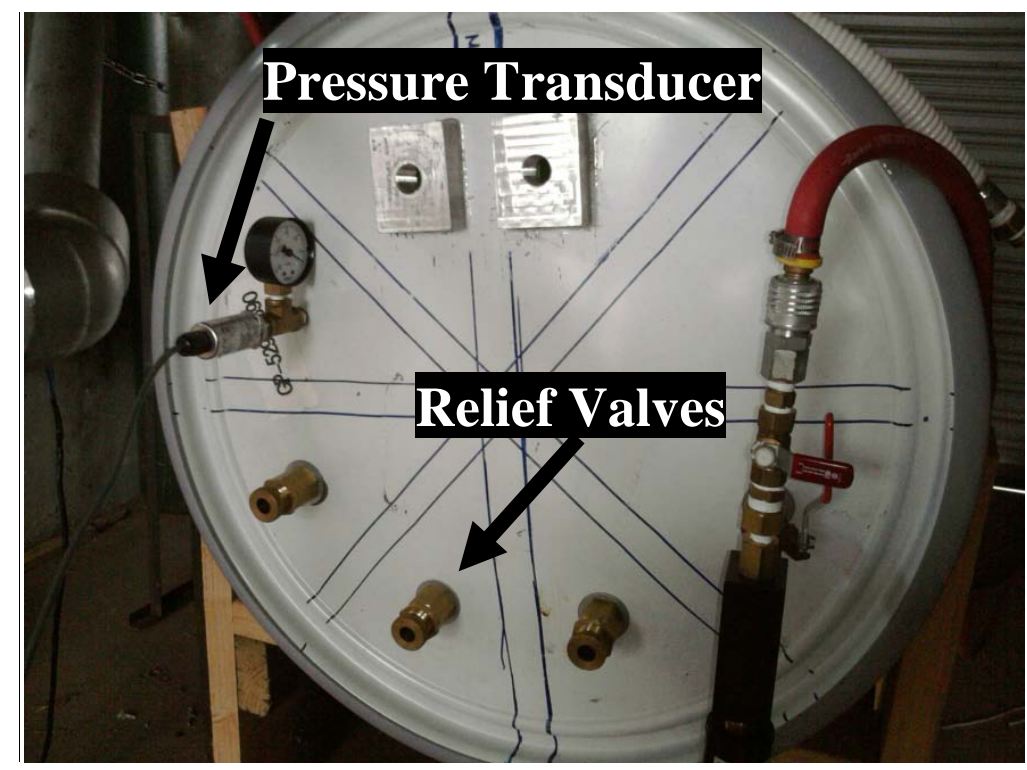

Figure 30: Inlet Configuration 2

A threaded fitting compresses the spring and determines the minimum pressure differential required to open the valve. As the pressure differential increases the area of the relief flow increases relative to the spring rate. This constantly changing mass flow relieving the plenum adds another transient component to the transient secondary flow of the plenum. With this system there was no absolute way of determining the minimum pressure that would be achieved during a test. In order to mitigate this, a new system was developed that would actuate a relief valve remotely at a setting desired by the operator. 
The relief valves were used to ensure the integrity of the plenum and verify the external load capacity the structure could support. After several tests, the relief valves were determined to be insufficient for proper relief of the system as well as disrupting the minimum secondary pressure capability of the system. A redesign of the relief system was done for the third and final iteration of the intake system, a solenoid ball valve was used to actuate the relief system is the test required it. The valve was attached to the face of the plenum as shown in Figure 31.

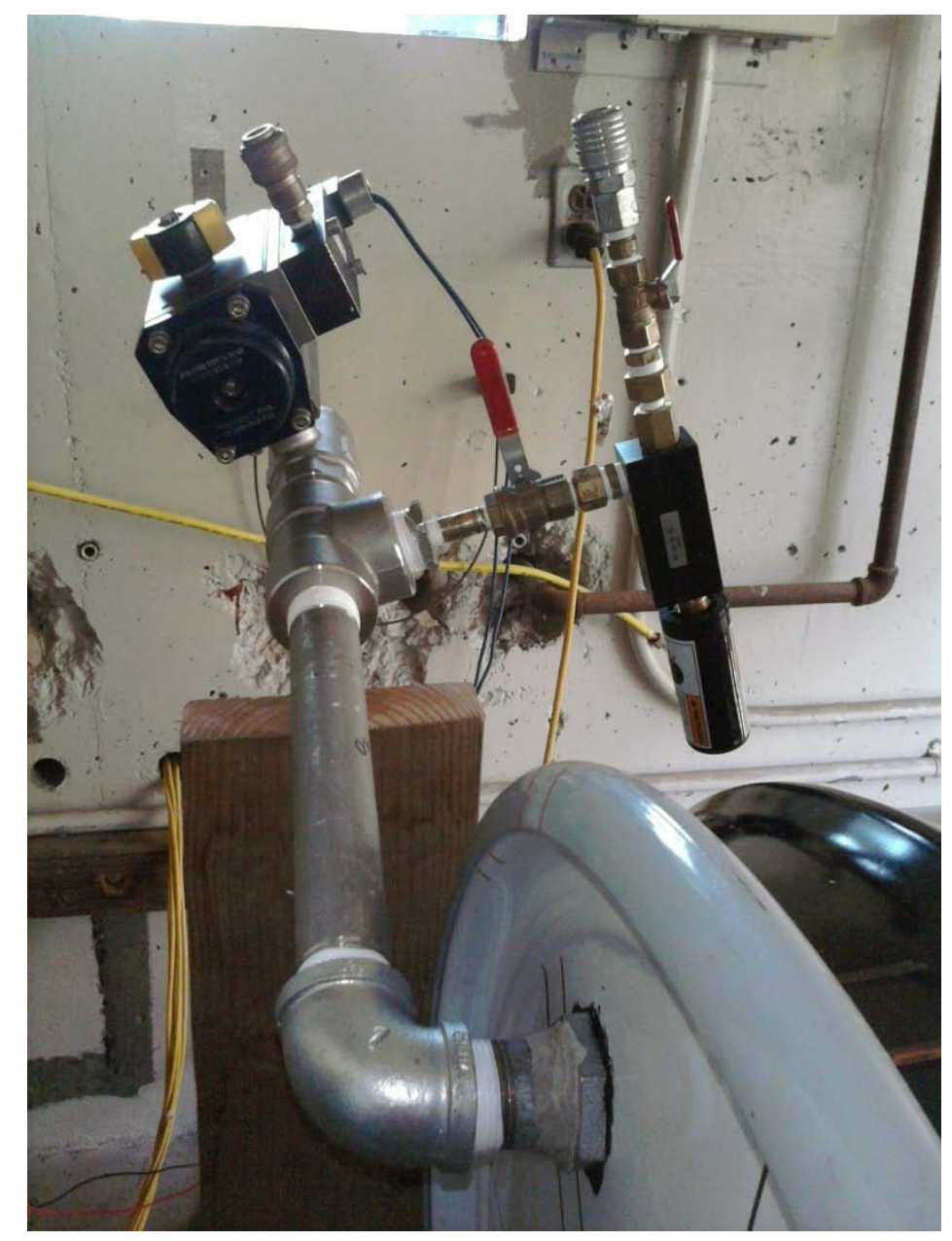

Figure 31: Relief System, Inlet Configuration 3

The solenoid valve for the relief system of the plenum was designed to be actuated when a predetermined minimum pressure in the plenum was achieved. The pneumatic solenoid 
required 120 psi of compressed air supply and 110VAC power supply. The solenoid was attached to a 1" NPT stainless steel ball valve. In order to remotely operate the valve at a point during the test determined by the operator, a small control circuit was designed. The goal of this system was to allow the operator to control the actuation of the solenoid during a test while observing the real-time pressure data from the plenum on the LabView display.

The circuit uses a relay to control AC power delivery to the solenoid valve. The relay coil is powered by a 10VDC power supply. A MOSFET is used to control the 10VDC Power supply to the relay. The MOSFET requires a +5VDC signal at the gate to allow the 10VDC to power the relay. A small DC power supply was used to send a +5VDC signal through a switch to the MOSFET gate, the amperage requirement to operate was reduced by adding a $4.7 \mathrm{kOhm}$ resistor between the gate and source of the MOSFET. Power to the valve is controlled by the operator with the +5VDC switch actuation.

All of the pressure and temperature measurements from the instrumentation on the test apparatus are observed during the test by the operator. Vital instrumentation observed were the primary chamber pressure, plenum pressure and wall pressures, these channels were specifically chosen to monitor hardware health. Before a test, the operator determines a minimum limit of the plenum pressure for that test based the design limits, condition and previously achieved pressures of the plenum. This allows the operator to control the flow conditions of the secondary duct accurately for any test. If the plenum achieved a pressure lower than the predetermined limit, the operator could actuate the secondary relief system with the +5VDC switch. 


\subsection{Data Acquisition}

To measure pressure in the system, Omega PX-302 general purpose pressure transducers were used. The transducers required a 10VDC input, and had a 0-100mV signal output. The pressure transducers operate at $1 \mathrm{kHz}$ with a $1 \%$ full scale reading error ${ }^{17}$. The location of the instrumentation during testing is shown in Figure 32.

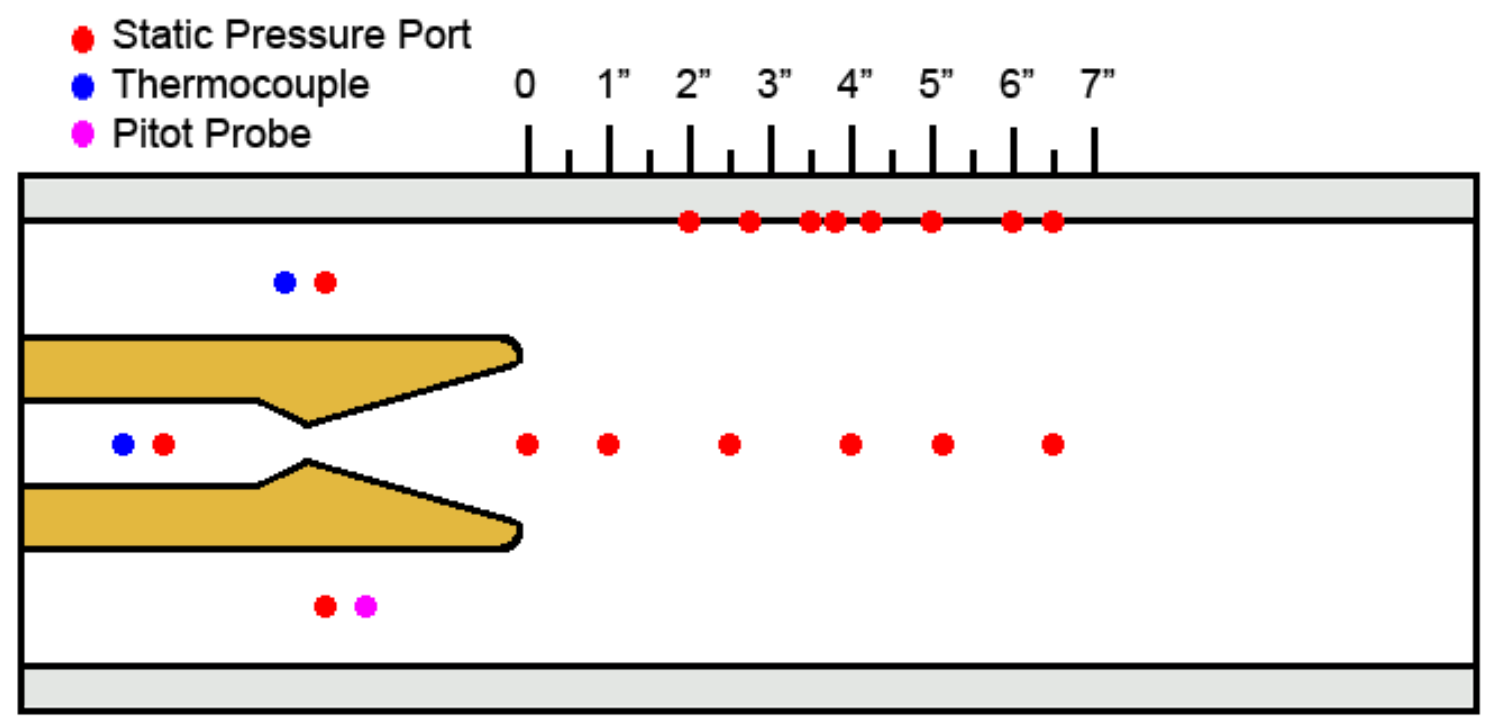

Figure 32: Instrumentation Locations

Pressure measurements were used to observe primary and secondary flow characteristics upstream of the mixing duct as well as in the mixing duct. 0-3000 psig model pressure transducers recorded the pressure of the buffer tank as well as stagnation pressure of the primary flow combustion chamber. One 0-50 psia pressure transducer measured the static pressure of the nozzle exit to directly calculate the Mach number of the flow. Three 0-15 psia model pressure transducers measured the stagnation and static pressure of the left secondary duct, and static pressure of the right secondary duct, upstream of the mixing duct. These measurements were used to calculate the mass flow rate of the secondary flow. One 0-50 psia pressure transducer measured the pressure of the plenum, upstream 
of the secondary inlet. The plenum pressure transducer measures stagnation pressure inside the plenum on the front face of the plenum as seen in Figure 30. Eight 0-50 psia pressure transducers measured the static pressure of the right wall of the mixing duct in varying locations from 2 inches to 7.215 inches past the primary nozzle exit. Five 0-50 psia pressure transducers measured the static pressure of the primary flow along the centerline of the mixing duct from 1 to 6.5 inches.

Temperature measurements of the primary and secondary flows were taken for mass flow rate calculations. A total of two type $\mathrm{K}$ shielded thermocouples were used that operate over the range from $-328 \mathrm{~F}$ to $2282 \mathrm{~F}$ with less than $4 \mathrm{~F}$ drift over the entire range ${ }^{18}$.

A National Instruments SCXI-1000 chassis provided power to a SCXI-1102 amplifier which was connected to an SCXI-1303 terminal block. Signals from the pressure transducers and thermocouples were routed to the terminal block and amplified resulting in an output signal to a laptop computer via a National Instruments PCMIA DAQ card 6063E. Each individual signal was identified based on the channel it was connected to on the terminal block. LabView, a data measurement program developed by National Instruments, was used to process and record signals from the instrumentation. A LabView program developed by Foster/Gist was used initially for early tests. Modifications to the code were made to change data resolution and reorganize the recorded data to an excel worksheet output file, Figure 33. 


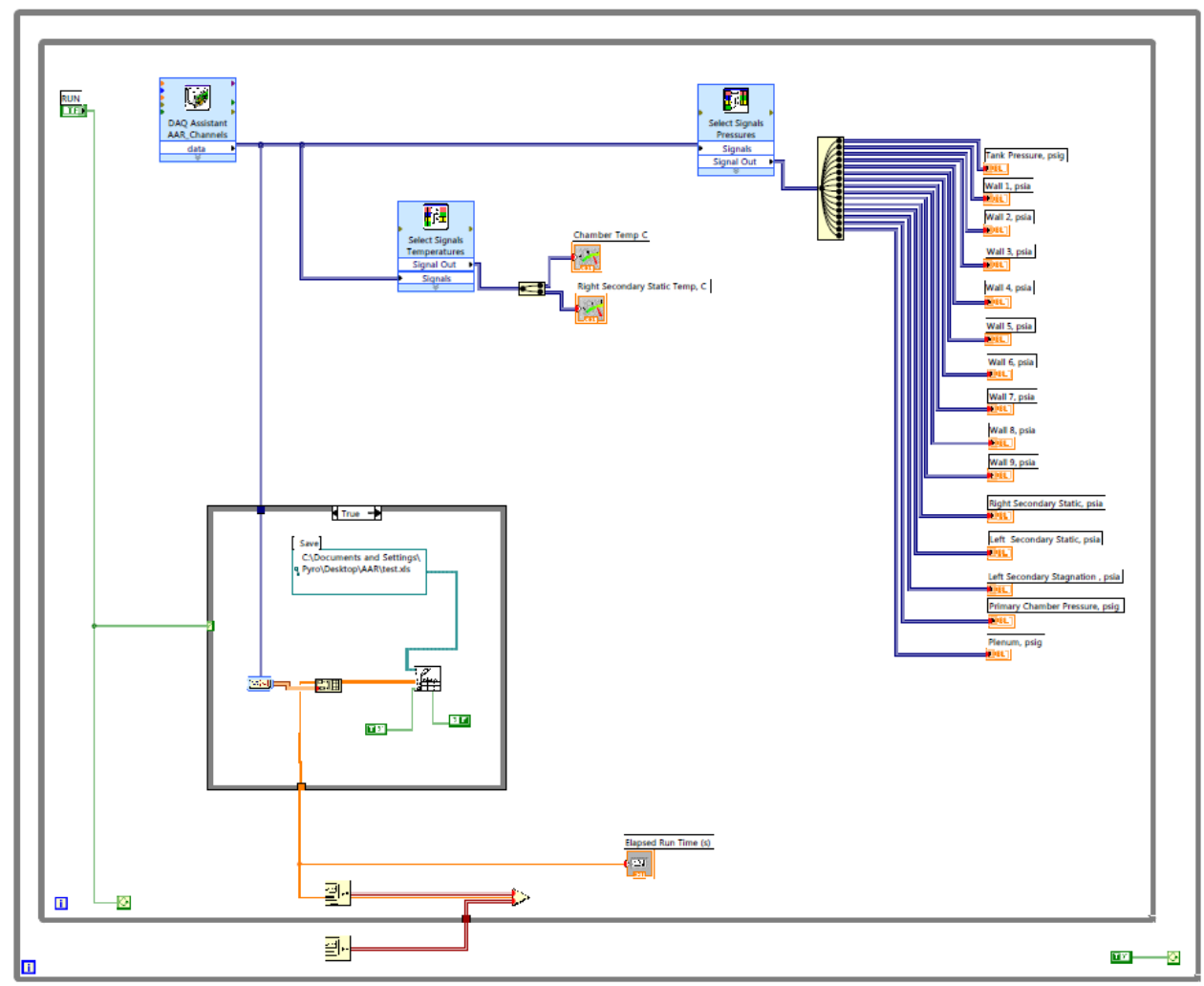

Figure 33: LabView Program .VI for Testing

During initial testing with data recorded at $500 \mathrm{~Hz}$, the same data rate as previous research $^{4,9}$, the output files would have large significant lapses in data as a result of the inability of the laptop to process significant samples per second. The sampling frequency observed by the code from the instrumentation is based on the capability of the instrumentation hardware and Data Acquisition hardware, while the samples processed and recorded to the output file are dependent on the processing power of the computer. In order to rectify these lapses in data during a test, the code output was rewritten to average the samples per sampling frequency. The result was a significantly smaller size of the output file, but a sample rate high enough to observe transient data recorded by the instrumentation and not choke the computer processor. The sample rate was $50 \mathrm{hz}$. 
A MATLAB code was developed to process and analyze the raw data excel file output from each test. The equations used for the analysis of the data are produced in section 6.2. The code also produced plots of the data used for analysis of the flow field. 


\section{Preliminary Testing}

The CPAAR was tested in four separated configurations during this test series. The first initial tests were done with the secondary ducts exposed to atmosphere. The next three segments of tests are broken up according to the iteration of the plenum intake system design, and subsequently the analysis of the data.

The tests where the secondary ducts were exposed to atmosphere were performed for hardware configuration and system health analysis purposes. For the high pressure ratio testing, an intake system was devised to connect the secondary flow ducts to a fixed volume plenum. Several issues arose with choking occurring upstream of the duct and producing turbulent flow in the secondary duct and mixing duct.

\subsection{Atmospheric Hardware Tests}

The atmospheric tests were completed initially for the re-integration and set-up of the hardware. The hardware for the air augmented rocket had been sitting in a box in the test cell for over 2 years, the test stand had been used by students for other projects and much of the instrumentation originally used had been broken, lost or used for other projects. After a period of re-assembly of the hardware and instrumentation, a series of tests were done to validate the hardware and data acquisition system was operational. These tests were done to work out problems in the system before the plenum was attached and high pressure ratio tests were done. Initially the rocket was operated at low primary mass flow rates to test the instrumentation and data output.

An initial series of eight tests (Tests 1-8) were completed to proof the system. Each test was done at a higher primary chamber pressure to validate the operation of the rocket. 
The primary maximum primary chamber pressure was controlled by the initial pressure set in the buffer tank. The tests conducted ranged from 500-1600 psi in the primary chamber. The data obtained from this testing was used to establish a basis for the performance of the CPAAR. The data also showed evidence of an asymmetry of the flow, the problem was mitigated by re-assembling and resealing the hardware to reduce leaks into the system.

\section{2. $\quad$ High Pressure Ratio Testing}

Initial testing of the CPAAR yielded valuable knowledge about the operation of the rocket and a list of items to be completed before high pressure ratio testing could be done. Once the initial issues with the CPAAR hardware and instrumentation were found and resolved, the high pressure ratio test series began. With the attachment of a fixed volume plenum to the secondary inlets, the total pressure of the secondary flow would decrease and produce high pressure ratio results.

The initial design of the plenum intake system for the secondary flow produced a choke point upstream of the secondary duct and was discovered during these initial hardware validation tests on the plenum. The choke point effectively meant that a higher pressure ratio was required to transition from a saturated case to a Fabri-choke case, or the minimum area of the secondary flow in the mixing duct, effectively increasing the effective pressure ratio requirement for the saturated case.

The second iteration of the plenum intake system was an attempt to increase the minimum area in the intake piping and further test the performance of the plenum. These tests saw a decrease in the pressure ratio requirement for the saturated case. Despite this, 
the non-isentropic area changes in the intake piping invalidated the isentropic and steady flow assumptions for the analysis.

The third iteration of the intake system was a complete redesign of the intake system in order to facilitate a steady and isentropic secondary flow. The square duct was effectively extended into the plenum such that the secondary ducts were now constant area ducts. The adjustable relief valves were removed and replaced with a remotely controlled solenoid valve. The solenoid valve would be actuated if the plenum reached a predetermined minimum pressure. This was done as a safety precaution initially to validate the hardware's ability to achieve low pressures. The relief valve was a 1” NPT ball valve. The relief valve was not actuated during formal data collection.

\subsubsection{Secondary Inlet Choking}

The performance of the first iteration of the plenum intake system was observed in the next ten tests (Tests 9-18). This series of tests and was done with the plenum attached to the secondary ducts. As mentioned, the development of the plenum was defined by several constraints. These tests were done at lower pressures to observe the rates at which the plenum evacuated and to observe any leaks in the system.

A few of these tests attempted using several materials as burst discs. Burst discs were used in the secondary inlets in an attempt to start the system while the plenum was at a lower pressure initially. The theoretical burst pressures were calculated for multiple materials including plastic and aluminum of varying thicknesses. Although burst disc theory suggested this method was possible, small leaks in the two separate ducts would result in either disc bursting before the other, resulting in asymmetrical flow. This 
method was scrapped in lieu of using a block at the end of the mixing duct to achieve low pressures in the system before the test.

By putting a block at the end of the mixing duct, the entire apparatus be evacuated to a vacuum before a test. The evacuation was done using a simple ejector vacuum pump supplied by 120 psi air. The high pressure of the primary flow would push the block out of its position at the end of the mixing duct at the start of a test. The vacuum level obtained in the system via evacuation pre-test was not low enough to have a significant effect on total pressure ratio achieved during tests of this configuration.

The second iteration of the secondary inlet ducts were constructed and tested in Tests 1922. The attempt at the second iteration of the secondary inlet configuration involved a series of items to increase the size of the duct to reduce the probability of the choking occurring upstream of the mixing duct. For Tests 19-22, a block was placed at the end of the mixing duct, and the entire apparatus was evacuated prior to a test. During Test 19, the epoxy supporting the pitot probe cracked and caused the probe to rotate, resulting in an incorrect measurement of flow in the left secondary duct. Tests 20-22 achieved high pressure ratios, but were later determined to be un-useable because of the turbulent flow created by the inlet configuration.

\subsubsection{Secondary Inlet Constant Area Duct Configuration}

The operation of the third configuration of the plenum was fundamentally different than the two previous iterations of the intake. Relief valves no longer have varying opening areas based on pressure differential. The relief valve is a pneumatic solenoid valve operated remotely via a switch in the control room. The relief valve can be actuated when the pre-determined minimum pressure in the plenum is reached. 
Mass flows are much higher in these tests, which was a result of the removal of the upstream choke point in the inlet ducts. For several tests, the relief valve was actuated, to understand the operation of the new configuration. The primary flow could entrain much more mass from the secondary with the larger inlets. The relief valve was actuated at 5 psia for tests 23-26.

Tests 23 and 24 were low pressure tests of the third inlet configuration. The test was used to verify the seals of the plenum during operation. The mixing duct was modified with the addition of 4 wall pressure ports, at 5.5”, 6.0”, 6.5”, and 7.125” as well as static temperature in the right secondary duct.

Test 25 was the first high pressure test of the new inlet configuration, the test was also to acclimate the operator to opening the relief valve at a proper time, ensuring that the relief valve could be actuated in a timely fashion, before a catastrophic failure of the plenum could occur. Addition of thermocouple in the secondary allows for direct secondary mass flow calculations of the secondary flow. Previous tests had used ambient temperature for the calculation.

Test 26 ended up being the highest pressure ratio test ever recorded. Along with Test 25, these tests were the actual high pressure runs of the current inlet configuration. The operation of the third configuration of the plenum is fundamentally different than the two previous iterations of the intake.

The primary flow valve opened very slowly, it resulted in a slow gradual pressurization of the chamber, which allowed the plenum to achieve a very low pressure. The characteristics of the flow produced only a small amount of time where the primary flow 
was choked. The difference between a faulty and normal operation of the primary valve is shown in Figure 34 and Figure 35.

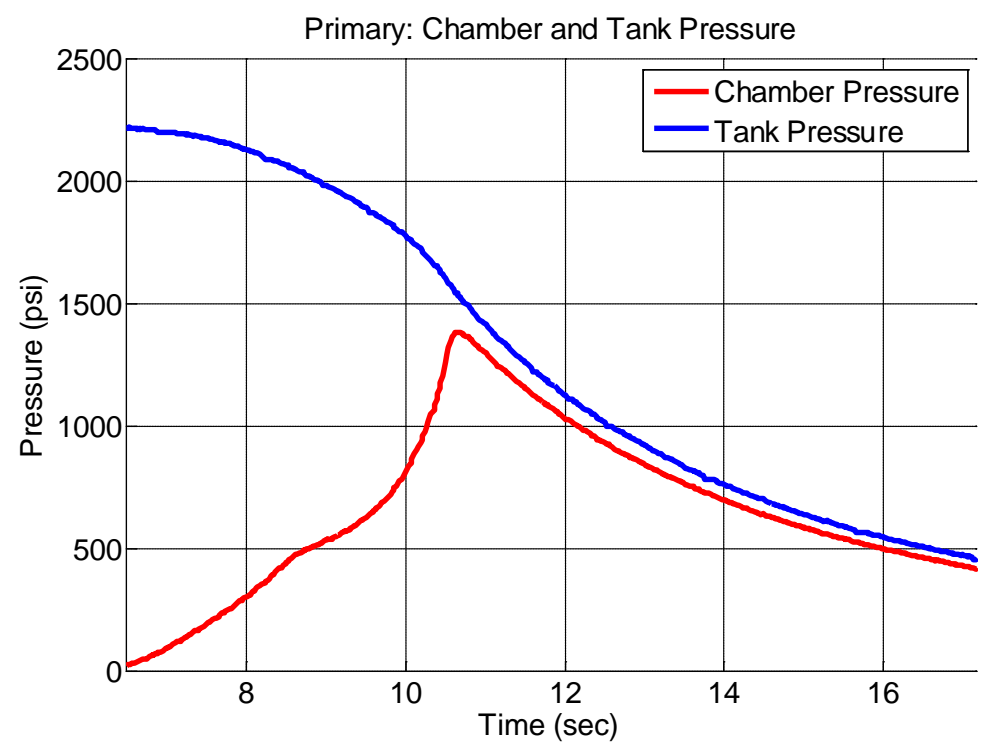

Figure 34: Faulty Primary Flow Valve Operation

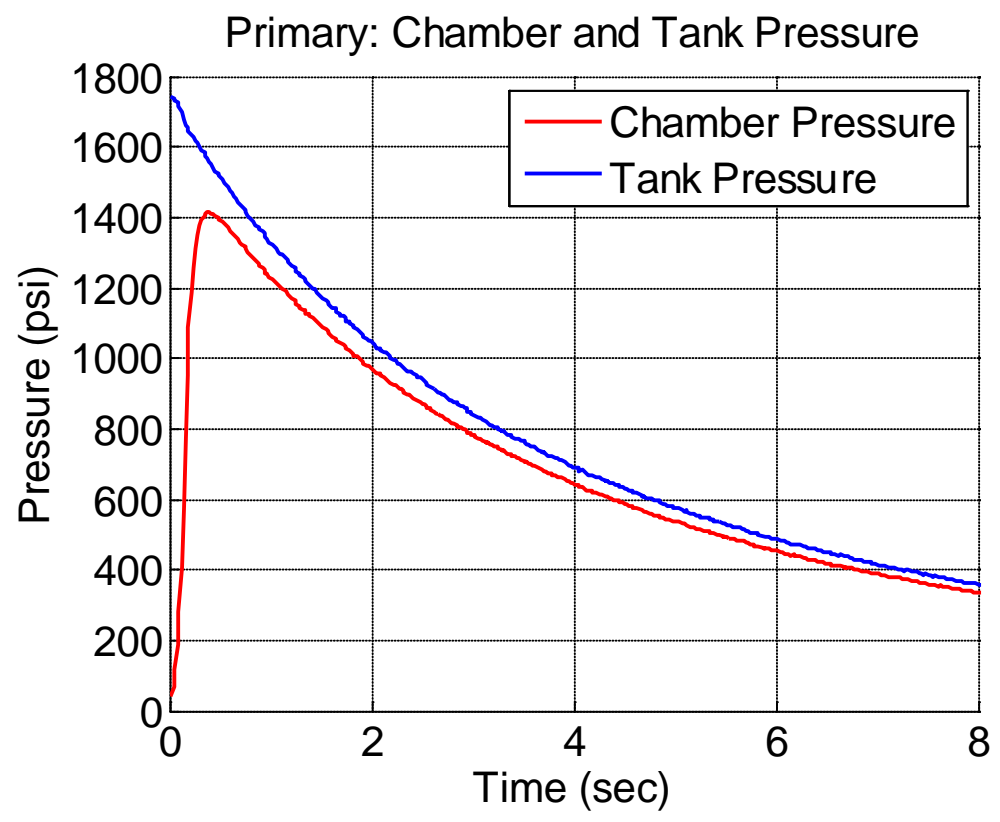

Figure 35: Normal Primary Flow Valve Operation

Despite the incredibly high pressure ratios achieved in this test, there was no indication in the data or video that a Fabri-block case was achieved. This is potentially the result of 
leaks in the wall pressure transducer ports that were not used and sealed with electrical tape. On the next test, the unused ports were be resealed with putty sealant. The slow actuation of the primary valve is shown in the figure below.

There were two issues that resulted from the tests. The first issue was related to the minimum plenum pressure that can be achieved during a test of the secondary flow. The problem during these tests was that the relief valve for the plenum was actuated at a predetermined pressure by the operator to ensure the structural integrity of the plenum. By the operator physically relieving the pressure, the highest pressure ratio potentially available during those tests was not able to be observed because the flow was interrupted before maximum pressure ratio could be achieved. The second issue was the possibility of leaks along the mixing duct wall pressure tap locations. These would bleed ambient pressure air into the mixing duct along the wall, creating an area where the primary plume would be unable to expand to.

To rectify the issues, for Tests 27 and 28 the leaks were sealed with adhesive putty and the tests run at lower pressures and not include actuation of the plenum relief valve. Both tests were run without operation of the relief valve and a base pressure was achieved. The minimum plenum pressure during both of these tests was 5.4 psia.

Test 29 was an attempt to achieve higher pressure ratios than previously before with the current intake set up as well not actuating the relief valve. By not opening the relief valve the plenum would be allowed to reach its minimum pressure value based on the conditions of the primary flow. The test produced data that appeared to be more stable than Tests 27 and 28. At this point it was determined the formal testing of the rocket 
should begin and successive tests, depending on post-test data validation, would be used for formal testing analysis.

Tests 30 and 31 were like Test 29 but with greater leak protection. To ensure there were no leaks in the system, sealant was applied to all part connections on the thruster. These two tests were planned to achieve the highest pressure ratio and the lowest plenum pressure. The plenum relieve valve was not actuated. These tests achieved the highest pressure ratios to date, with the highest degree of data integrity as well. The data from these tests are used in the discussion of the results.

To observe the shock structure of the primary flow centerline pressure ports were added to the system. The Schlieren video capability was removed. During the Tests 32 and 33, the relief valve was not actuated, and the plenum was able to achieve the minimum point based on ejector performance only.

The bottom glass plate of the thruster was replaced with a solid aluminum piece that had pressure ports along the centerline. The centerline pressures allow the observance of the primary flow and direct calculation of the Mach number of the primary flow at the nozzle exit of the thruster. During re-assembly, a crack in the top glass plate propagated across, spanning the width of the mixing duct.

During Test 32, there was a failure in the primary flow valve actuation causing the valve not to open completely which resulted in an extended duration low flow rate test. The data is not representative of nominal operation due to the low flow rate of the primary flow and lower chamber pressure. The valve failure was reflected in the observable slow pressure rise of primary chamber and lower than expected peak chamber pressure during the test. 
After Test 32, for Test 33, the valve was inspected and actuated to assure proper operation. The test was done with the same set up as Test 32, with the new instrumentation to obtain primary flow centerline data. The test produced data which verified the how the shock structure of the primary flow collapses after the maximum pressure ratio. 


\section{Formal Testing}

Once the initial issues associated with the operation of the CPAAR, it was determined to begin the formal testing of the rocket. The tests used for the formal testing analysis were Tests 29-33. The testing consisted of three tests which produced very high pressure ratios, and two tests producing valuable primary shock structure data. The maximum pressure ratio achieved over the formal testing period was 220. The wall pressures recorded during the formal testing provided valuable insight to the location and movement of the Fabri-choke location of the secondary flow.

\subsection{Test Operation Procedure}

The CPAAR was tested at the Cal Poly Aerospace Engineering Departments Test Cell. The control room is separated from the test cell by 12 inches of concrete with a 4-inch thick tempered glass window to see inside the cell. During testing, the 10 fingers, 10 toes rule was in affect and a minimum of two personnel were required for operation.

Each test used two high pressure nitrogen cylinders. Depending on the tests and availability of cylinders, the pressure inside the cylinders varied from 1500 psi to 2600 psi. The primary flow valve position was ensured in the "OFF" position. The lower pressure of the two were equalized with the buffer tank and then isolated, and subsequently the high pressure tank was equalized with the buffer tank. Once the tank was pressurized, personnel were removed from the test cell and the area around the test cell was cleared. Dependent on the test, the shadowgraph camera was operational. The LabView data acquisition was started as the countdown initiated. The primary flow valve was energized and the test was initiated. 
Once the primary flow valve was open, nitrogen pressurized the primary chamber and expanded through the nozzle into the mixing duct and ejected to ambient air at the mixing duct exit. The primary plume creates a shear force along the plume which entrains air from the plenum through the secondary ducts into the mixing duct. Depending on the actuation of the primary valve, the first portion of the test is a transient startup region based on the pressurization of the primary chamber. Then the primary chamber begins to discharge until it returns to ambient pressure. For a period of time while the momentum of the primary flow is high enough a steady shock structure is formed in the primary plume producing a quasi-steady quality to the flow. The flow rapidly under-expands and maintains the steady shock structure. As the flow becomes optimally expanded and subsequently over-expanded, the chamber pressure falls low enough such that the flow is no longer able to maintain supersonic flow and the shock structure collapses.

\subsection{Data Post-Processing Methodology}

The raw data recorded by LabView was output to an excel file, and then a MATLAB file was developed to reduce, identify and analyze the data. Data during formal testing was recorded on 19 parallel channels at a rate of $50 \mathrm{~Hz}$. Although the system is capable of recording at $1000 \mathrm{~Hz}$, this rate was determined unnecessary during validation tests for the purposes of this experiment.

Numerous calibrations were performed on the pressure transducers, but it was observed that during ambient operation, offsets of separate pressure transducers would be as large as $0.5 \mathrm{psi}$ on certain pressure transducers. Over time, pressure transducers and thermocouples lose accuracy, the variances increase over time and cyclic usage. To rectify these offset variances, the recorded pressure and temperature values were 
normalized to the ambient conditions at the time of that test. This was achieved by subtracting the recorded ambient condition and adding a standard ambient value to increase the accuracy of the pressure transducer reading along the wall of the mixing duct.

The raw data recorded during tests consisted only of pressure and temperature. As these were the only values directly measured, several other parameters that could assist in the analysis of the flow-field were calculated indirectly. The equations and methodology for these parameters are presented as follows:

The flow in the secondary inlet ducts was assumed to be isentropic and compressible. The Mach number of the secondary duct was calculated using the recorded values of stagnation pressure $\mathrm{P}_{\mathrm{s}}$, and static pressure $\mathrm{p}_{\mathrm{s}}$, and was derived from the isentropic equation for compressible flow.

$$
M_{S}=\sqrt{\frac{2\left(\left(\frac{p_{s}}{p_{s}}\right)^{\frac{\gamma-1}{\gamma}}-1\right)}{\gamma-1}}
$$

In the secondary duct, flow is considered compressible during saturated and Fabri-choke conditions. Although stagnation pressure and static pressure are measured, only static temperature of the secondary duct is measured. To complete the mass flow calculation, stagnation temperature of the secondary flow is required; this is also done through the isentropic compressible flow relations.

$$
T_{s}=t_{s}\left(1+\frac{\gamma-1}{2} M_{s}^{2}\right)
$$


The mass flow rate of the secondary is determined by the compressible mass flow rate equation and is dependent on the stagnation conditions and Mach number of the flow. The mass flow rate is calculated in the secondary duct at the static pressure location separately in both ducts.

$$
\dot{m}_{S}=\frac{A_{s} P_{s}}{\sqrt{T_{s}}} \sqrt{\frac{\gamma}{R}} M_{S}\left(1+\frac{\gamma-1}{2} M_{S}^{2}\right)^{-\frac{\gamma+1}{2(\gamma-1)}}
$$

By calculating the mass flow rate at the pitot-static probe, the mass flow rate calculation is solely based on the fluctuations of the total and static pressure of the secondary flow. The system has an observed asymmetry as shown previously ${ }^{4}$, to help understand the mass flow rate of the secondary flow, the mass flow rate is also calculated using the stagnation pressure of the plenum and the static pressure recorded in the left and right secondary ducts. This allows for mass flow of each individual duct to be calculated, but does not account for losses in the secondary inlets.

The choking area of the secondary flow can be determined by establishing a set of conditions to the above equation known for a choked flow. Once the primary flow becomes under-expanded, the Fabri-choke area can be determined by solving for the area, $\mathrm{A}_{\mathrm{s}}$ and setting the Mach number, $\mathrm{M}_{\mathrm{s}}$ to 1 .

$$
A_{S}=\frac{\dot{m}_{s} \sqrt{R T_{s}}}{P_{s} \sqrt{\gamma}}\left(\frac{\gamma+1}{2}\right)^{\frac{\gamma+1}{2(\gamma-1)}}
$$

The static pressures along the mixing duct wall are recorded in locations from 2 to 7 inches downstream of the nozzle exit on the right mixing duct wall. With the assumption of a compressible isentropic secondary flow we can use the derived equation below to calculate the Mach number at each location along the wall. This assumes that the flow 
interacting with the wall is the secondary flow. The static pressure is recorded by a pressure transducer at the specific wall location, while the stagnation pressure is recorded in the plenum.

$$
M_{\text {wall }}=\sqrt{\frac{2\left(\left(\frac{P_{s}}{p_{\text {wall }}}\right)^{\frac{\gamma-1}{\gamma}}-1\right)}{\gamma-1}}
$$

The mass flow rate of the primary flow is determined by directly measured values of pressure and temperature. The assumption of isentropic flow in the primary states the pressure and temperature in the primary combustion chamber are at stagnation conditions, or zero velocity. The critical mass flow rate is dependent on these measured values and known properties of the gas and dimensions of the thruster.

$$
\dot{m}^{*}=\frac{P_{0} A_{t} \gamma}{\sqrt{\gamma R T_{0}}}\left(\frac{2}{\gamma+1}\right)^{\frac{\gamma+1}{2(\gamma-1)}}
$$

The Mach number of the primary flow was also calculated using equation 9 with the stagnation pressure of the primary chamber and the static pressure at the pressure ports along the mixing duct centerline.

\subsection{Experimental Error Analysis}

To determine the error in the calculated values used for analysis, the error in the instrumentation had to be determined and compiled to get to the final calculated values. Two types of error were considered, systematic error and random error. With relative accuracy, the uncertainty of the calculated values can be determined and used for analysis. 
Measurement of the raw data was done by the pressure transducers and thermocouples as described in the apparatus section. The Omega pressure transducers have a maximum uncertainty of $0.25 \%$ of the full scale reading ${ }^{17}$. The Omega thermocouples have less than 4 degree $\mathrm{F}$ drift over the full scale. The initial calibration of the thermocouples was performed using an ice bath with a calibrated mercury thermometer. The pressure transducers were calibrated using a steady-state vacuum ejector system and a certifiedcalibrated precision-accurate 0-30 in Hg vacuum gauge.

The measurements of physical dimensions of the apparatus also have an error associated with them. The least scale reading (LSR) associated with these dimensions was dependent on the calipers used to measure each dimension. The uncertainty of a quantity dependent on a physical dimension utilized the LSR in the error analysis.

A Pythagorean sum, $\Delta Z$, used the calculated experimental uncertainty of each measured value to calculate the total propagated uncertainty in the analyzed variables.

$$
\Delta Z=\sqrt{\left(\frac{\partial F}{\partial A}\right)^{2}(\Delta A)^{2}+\left(\frac{\partial F}{\partial B}\right)^{2}(\Delta B)^{2}}
$$

The total uncertainty associated with each quantity is represented in the following table as a percentage. 
Table 1: Experimental Uncertainty

\begin{tabular}{|c|c|c|c|c|}
\hline & Quantity & Symbol & Uncertainty & Standard Deviation \\
\hline \multirow{4}{*}{ 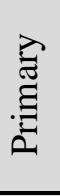 } & Density & $\rho_{\mathrm{p}}$ & $0.06 \%$ & $0.05 \%$ \\
\hline & Velocity & $V_{p}$ & $2.03 \%$ & $0.23 \%$ \\
\hline & Mach Number & $\mathrm{M}_{\mathrm{p}}$ & $1.18 \%$ & $0.02 \%$ \\
\hline & Mass Flow Rate & $\dot{\mathrm{m}}_{\mathrm{p}}$ & $0.31 \%$ & $0.28 \%$ \\
\hline \multirow{5}{*}{ 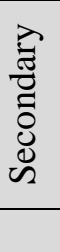 } & Density & $\rho_{\mathrm{s}}$ & $1.76 \%$ & $0.07 \%$ \\
\hline & Velocity & $\mathrm{V}_{\mathrm{s}}$ & $0.2 \%$ & $0.07 \%$ \\
\hline & Mach Number & $\mathrm{M}_{\mathrm{s}}$ & $0.35 \%$ & $0.14 \%$ \\
\hline & Mass Flow Rate & $\dot{\mathrm{m}}_{\mathrm{s}}$ & $2.95 \%$ & $0.18 \%$ \\
\hline & Entrainment Ratio & $E R$ & $2.96 \%$ & $0.18 \%$ \\
\hline
\end{tabular}

The uncertainty calculated on the experimental entrainment ratio was $2.96 \%$. The uncertainties in the calculations are not particularly large. The uncertainty in entrainment ratio data is much lower than previous research ${ }^{4,9}$ solely based on the different methods of calculation of the primary flow properties. The uncertainty associated with the data in the current research is based on the least scale readings (LSR) of the instrumentation and measurements. A plot of the uncertainty in the entrainment ratio calculation is presented in Figure 36. 


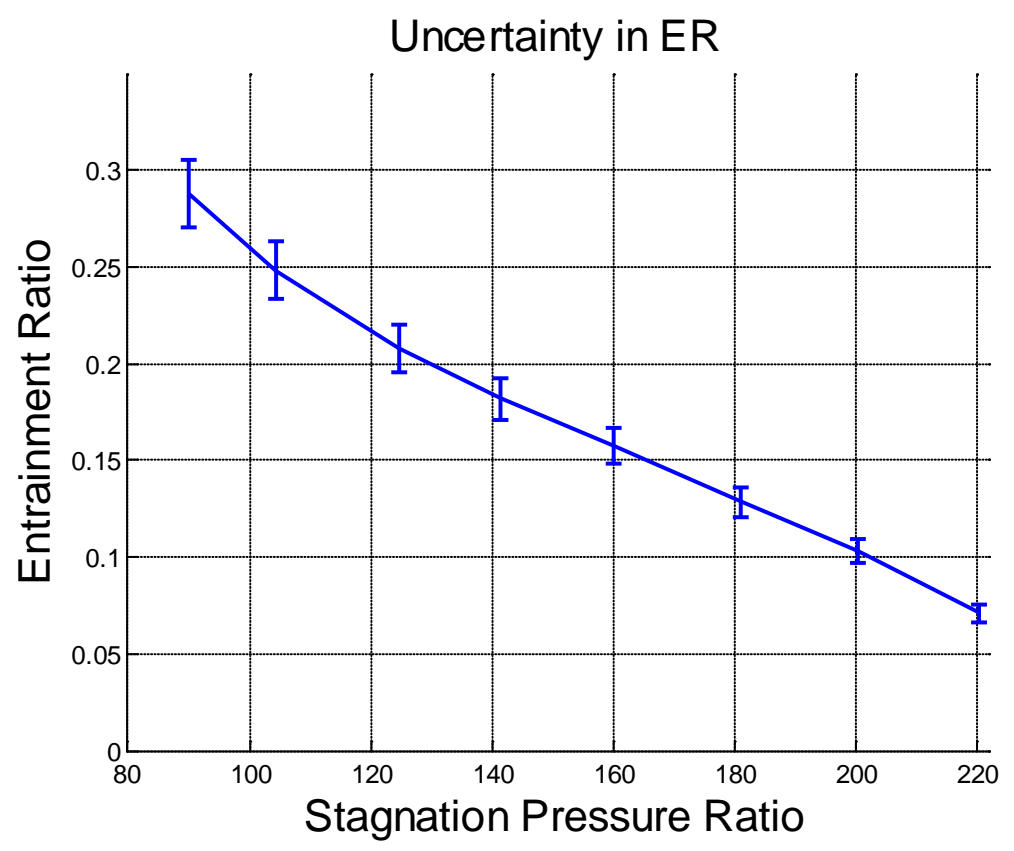

Figure 36: Test 30, Uncertainty in ER

At low pressure ratios the uncertainty produces a much larger range of potential values of ER. At a pressure ratio of 100 , the entrainment ratio may range $+/-0.03$. While at very high pressure ratios, such as those much closer to a potential Fabri-block case, the range of entrainment ratio based on the uncertainty is much lower, less than $+/-0.01$. With the low uncertainty of the data produced, the figures presented in the results do not have error bars associated with them. 


\section{Experimental Results and Discussion}

The original intent of the experiment was to study high pressure ratio flow-field activity in the mixing duct and attempt to achieve a Fabri-blocked case by modifying the test apparatus and compare with the simulation code. After the initial tests, the highest pressure tests with verifiable data were Tests 29 through 33. In this section, the results of those tests will be discussed and conclusions drawn from them. The tests are considered to have produced verifiable data by pre and post test inspection of hardware and instrumentation calibration.

From the results obtained with the Simulation code by Morham, with the inputs of primary and secondary flow conditions and pressure ratio, it was determined total pressure ratios greater than 230 would be required to achieve a Fabri-block. With the addition of a plenum on the secondary flow, repeatable runs of pressure ratios greater than 200 were produced. A blocked flow condition was achieved in the right duct during several tests. The blocking occurred as a result of an asymmetric breakdown of the primary plume causing the flow in the right duct to be entirely blocked as pressure increased in the opposing duct.

It was critical to determine a section of time which would be comparable between tests while satisfying the flow conditions for the assumptions used in the analysis of the data. Several events were observed across all of the tests: The pressurization of the primary chamber to a peak value and subsequent discharge, the evacuation of the plenum to a minimum value and subsequent pressurization, and the Pressure Ratio increase to a plateau and relatively slow decrease. All of these events were observed in different 
magnitudes as dependent on the maximum pressure ratio achieved and when the shock structure of the primary plume began to degrade during the tests.

The time from the actuation of the primary valve to time when the maximum pressure ratio is achieved was used to compare the data recorded during each test. This portion of time was used to plot the primary performance metric of supersonic air-to-air ejectors, the change in entrainment ratio as a function of stagnation pressure ratio.

Pressure Ratio and Entrainment ratio can be calculated via different locations, to keep the data consistent, the stagnation pressure of the plenum was used in calculations of PR and ER. The data obtained from the pitot probe is only applicable to the left secondary duct and due to losses in the inlet system; the pressure can be as much as $9 \%$ lower than the plenum, which falls outside the uncertainty of the data. The data presented in Table 2 is the performance of the CPAAR using plenum pressure data.

Table 2: Formal Testing PR and ER achieved

\begin{tabular}{|c|c|c|}
\hline & Maximum PR & ER @ PR_max \\
\hline Test 29 & 150.0 & $0.14(14 \%)$ \\
\hline Test 30 & 220.3 & $0.07(7 \%)$ \\
\hline Test 31 & 211.4 & $0.08(8 \%)$ \\
\hline Test 33 & 157.0 & $0.16(16 \%)$ \\
\hline
\end{tabular}




\subsection{Mixing Duct Results}

The Fabri-choke location in the mixing duct was found by the analysis of the data produced from the data reduction. By examination of the Mach numbers calculated along the wall over the period of the test, the movement of the Fabri-choke location is visible as the secondary flow achieves a Mach number of 1 at different locations. During the test, the change in the size and location of the Fabri-choke point can be analyzed relative to the pressure ratio achieved during the tests. The pressure ratio and the characteristics of the primary plume were also examined to understand the correlation between the maximum pressure ratio achieved and collapse and unsteadiness of the primary plume shock structure.

\subsubsection{Fabri-Choking}

Due to the nature of the apparatus, the flow is considered transient but quasi-steady. This assumption produces several interesting phenomena with relation to the establishment of either flow. The primary flow does not become fully established until a transient inflection point at which the secondary flow is established as well. For a period from the point when the secondary flow is established (past the startup transient) until the maximum pressure ratio is achieved, the primary flow undergoes transient behavior. To observe the Fabri-choking phenomena in the mixing duct, the static pressure recorded at several locations can be analyzed to show the movement of a shock in the mixing duct correlating the minimum area of the aerodynamic throat of the secondary flow. Figure 37 shows the inflection of the pressure ratio at its maximum during transient operation. 


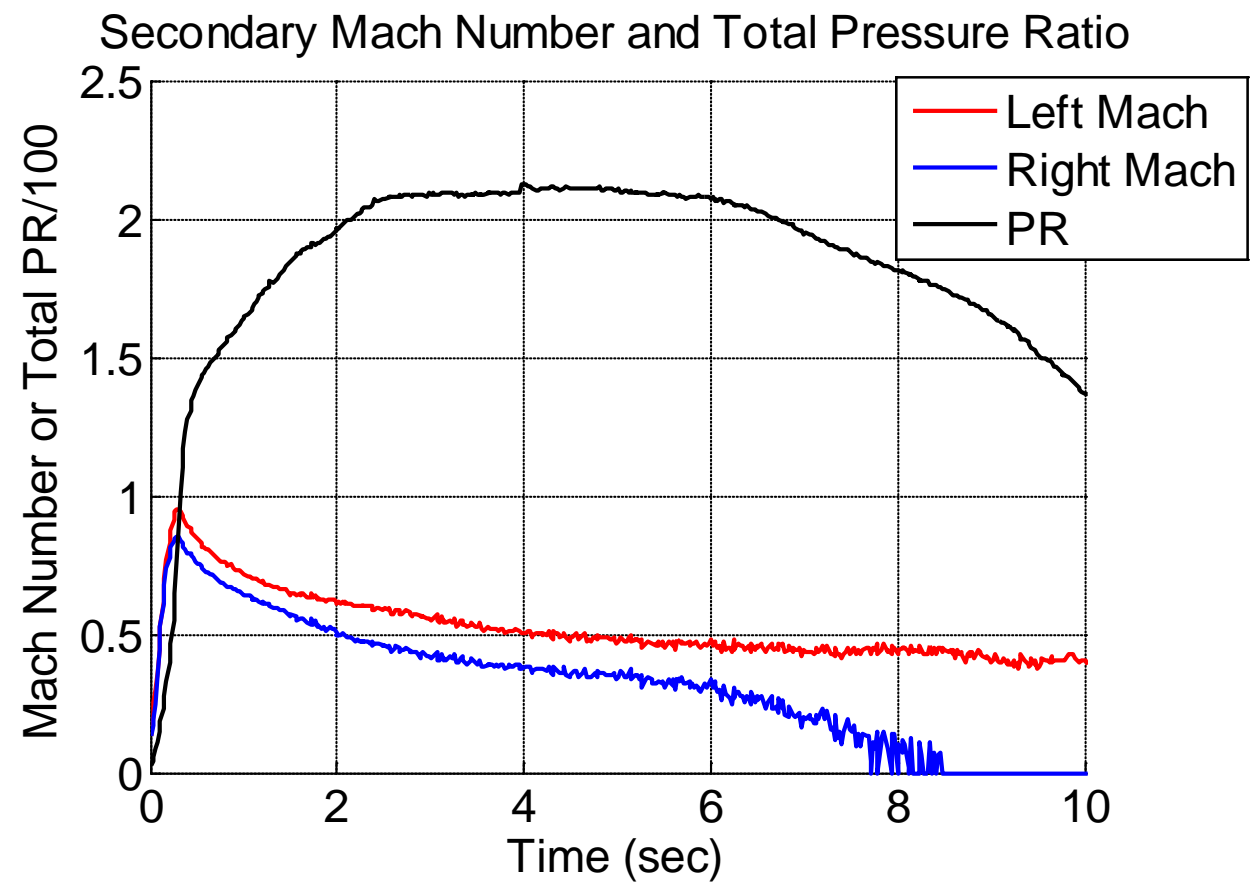

Figure 37: Test 31, Secondary Flow Mach number

At about 4 seconds, the maximum pressure ratio is observed. It is important to note the asymmetry of the secondary flow becomes more apparent as the difference in the Mach numbers becomes greater as the pressure ratio increases from roughly 100 to 211 ( 0.5 seconds to 4 seconds). As the pressure ratio increases during this time, the discharge transient period, the Mach number of the secondary flow at the secondary duct decreases. The reduction in speed suggests that a choke location downstream is reducing in size and moving downstream. As the primary plume increases in size, it reduces the size of the aerodynamic throat of the secondary flow causing a reduction of speed in the flow upstream observed at the secondary duct.

To observe the location of the aerodynamic throat, we can observe the Mach numbers recorded at static port locations along the wall. The static ports are along the right wall, 
and due to the asymmetry of the flow should be correlated to the Mach number and pressure ratio recorded in the right duct as shown in Figure 38.

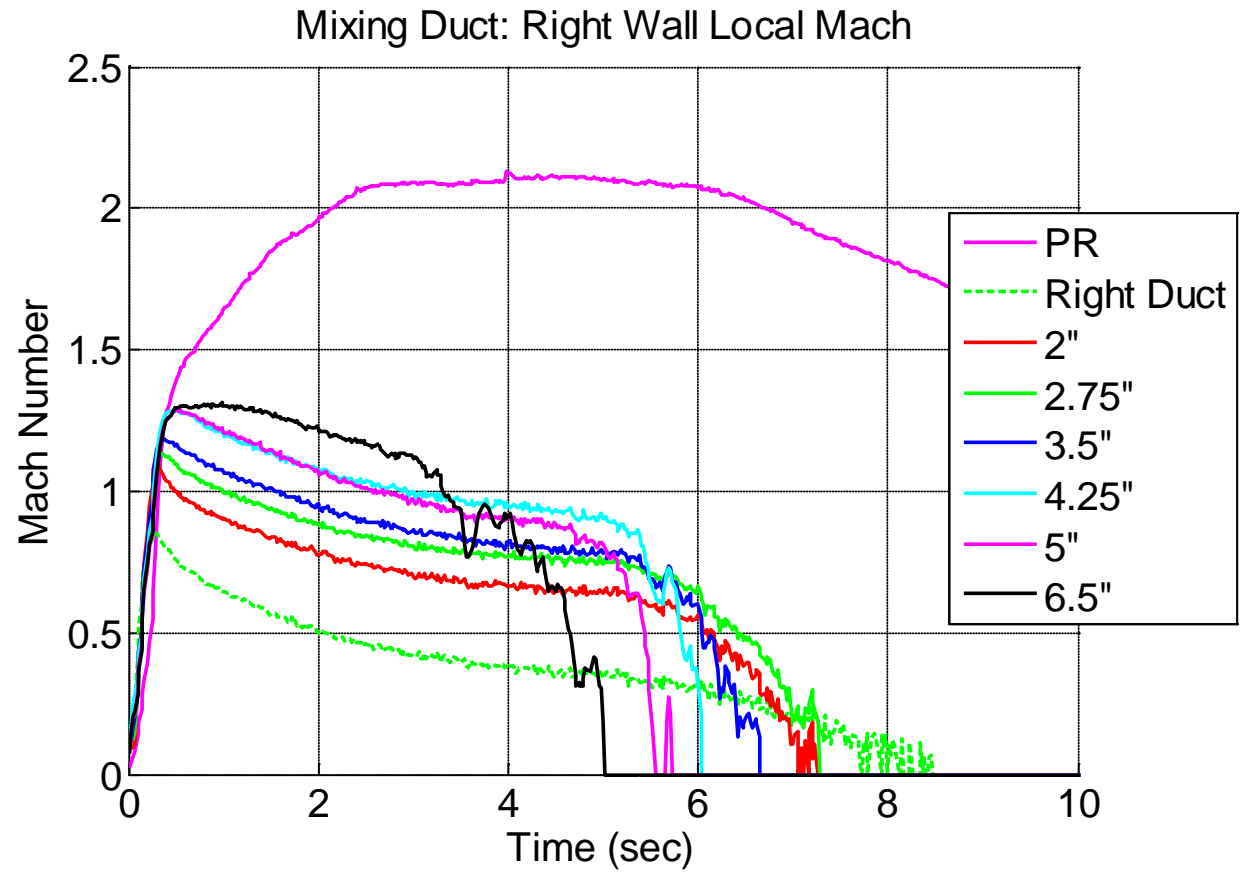

Figure 38: Test 31, Mach Numbers along Right Mixing Duct Flow-field

We can see each location decreases in Mach number as a function of time and increasing pressure ratio during the discharge transient region. The flows begin to collapse and reduce to zero after the point of maximum pressure ratio or maximum expansion of the primary plume, once the primary plume begins to collapse. But from the establishment of the flow to the maximum pressure ratio it is noticeable there is a constant trend among all of the locations. During a certain point in time, each location obtains a Mach number of 1 , representative of the location of the aerodynamic throat. The figure also shows the points upstream crossing the Mach 1 line before the downstream points, suggesting a shock moving downstream. To further observe this, we can observe the Mach number as a function of location shown in Figure 39. 


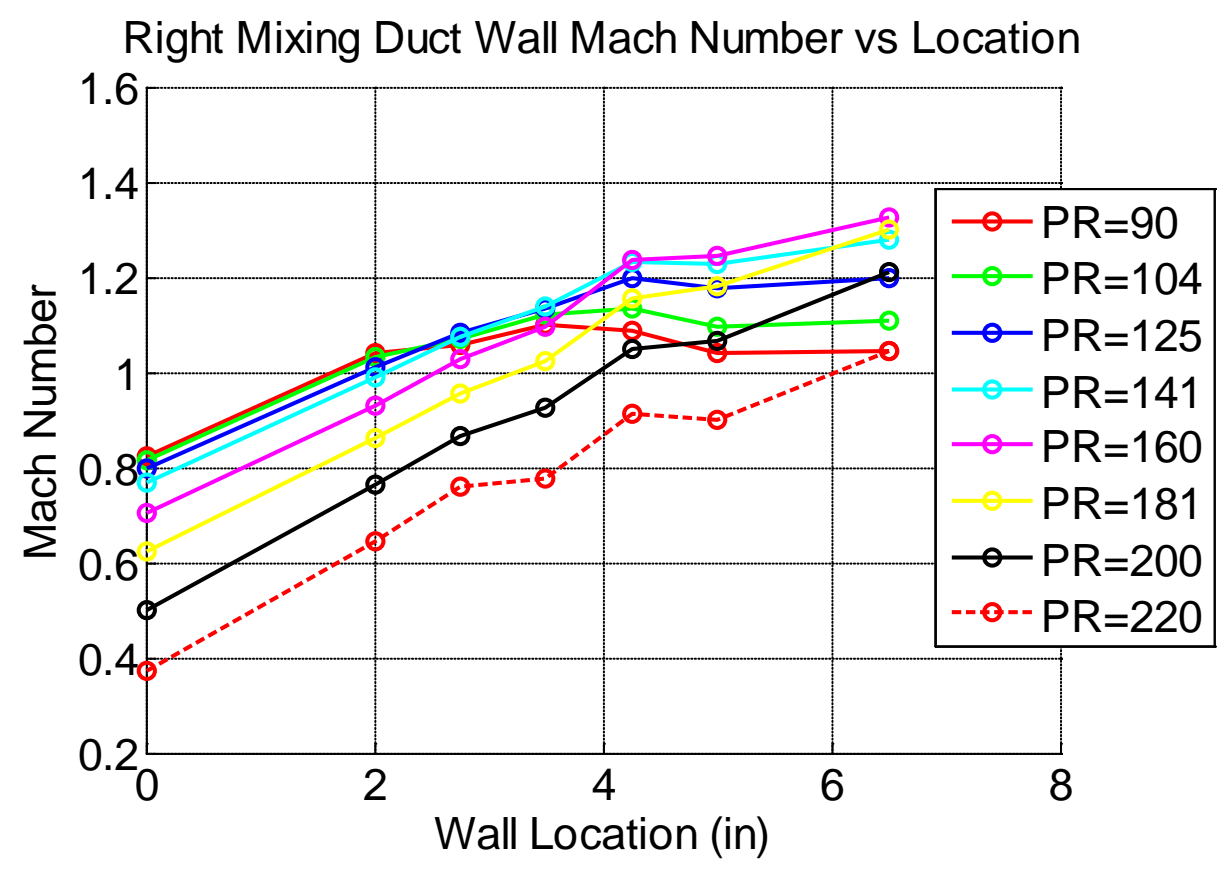

Figure 39: Test 30, Mach number vs. Location

Each line represents a different pressure ratio recorded during the period of ascending pressure ratio. It is obvious that as the pressure ratio increases, the location of the aerodynamic throat moves downstream. It is interesting to note, that the location of the shock moves downstream the slowest at lower pressure ratios and its rate of movement increases as the pressure ratio increases. The rate of change can be more easily observed in Figure 40, which shows the pressure ratio as a function of the wall choking location for different tests. 


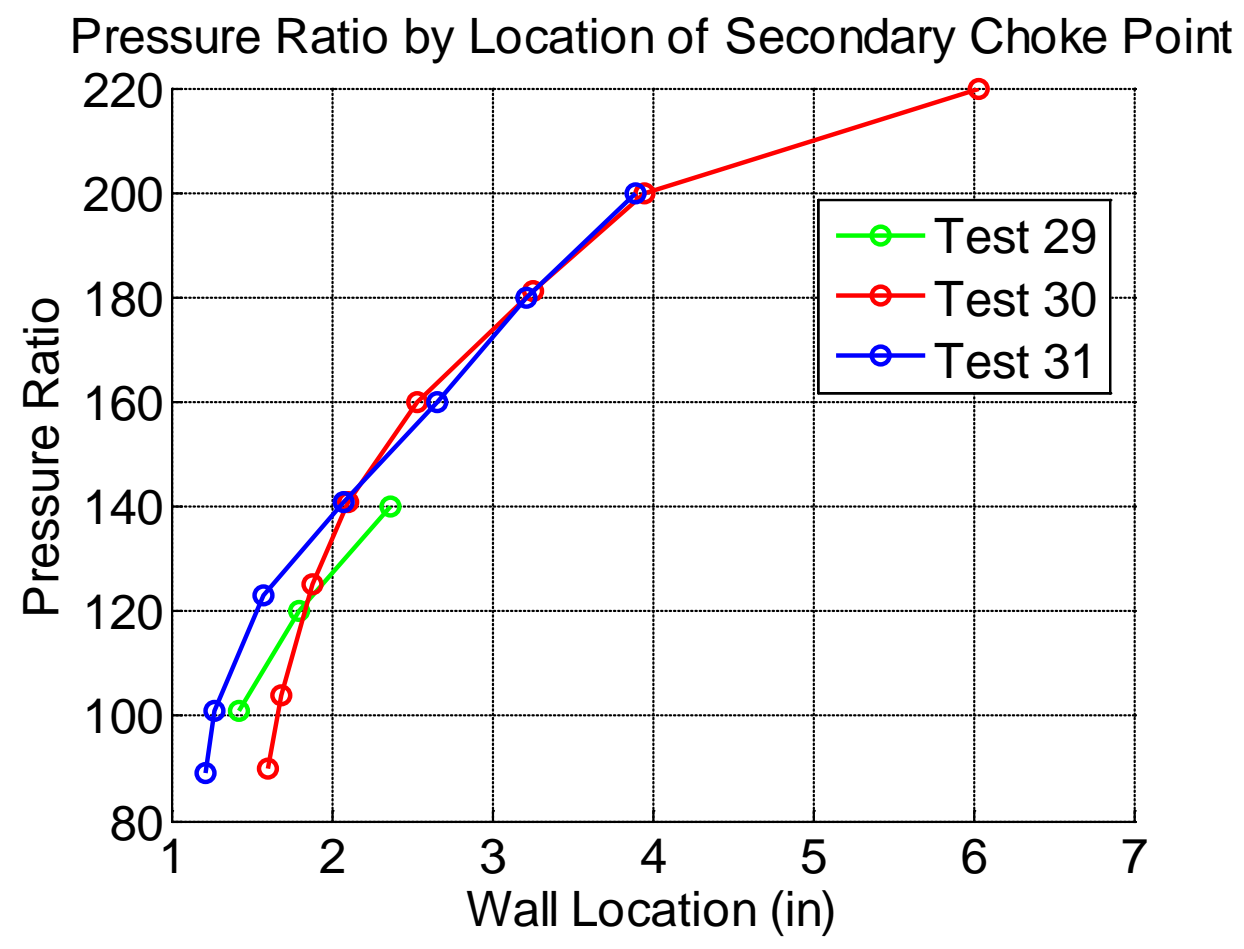

Figure 40: Wall Location of Secondary Fabri-choke Point

The rate of movement is represented in the change of slope of the line depending on the test. All of the tests follow the same trend. Test 30, was the highest pressure ratio test during the series of testing. The maximum pressure ratio achieved was 220 . The change in the aerodynamic choke location is very apparent, the choke location moves out to 6 inches past the exit of the nozzle at the highest pressure ratio, but is located at the same location for lower pressure ratios as other tests, as expected. For the purposes of easier analysis, the trends observed in Test 30 are assumed to be representative of extrapolated data of the other tests.

The rate of change of the location may be a function of the transient process of the flow, at the lower pressure ratios; the pressure ratio is increasing rapidly, while at about 140150, the pressure ratio begins to change at a much slower rate than before which is observed in Tests 30 and 31. The pressure ratio has a logarithmic relationship to the 
location of the secondary choke point. The Fabri-blocked case will occur at a certain pressure ratio, at a certain location along the wall of the mixing duct. If the pressure ratio continues to increase, the flow will theoretically begin to expand into the secondary ducts as a result of the growth of the recirculation zone at the nozzle lip.

The movement of the choke location downstream corresponds with expected results of observing a shock moving downstream at a rate relative to the change and rate of change in pressure ratio. We also expect as the primary plume expands, the aerodynamic choking area of the secondary flow should reduce while moving downstream; the data is represented in Figure 41.

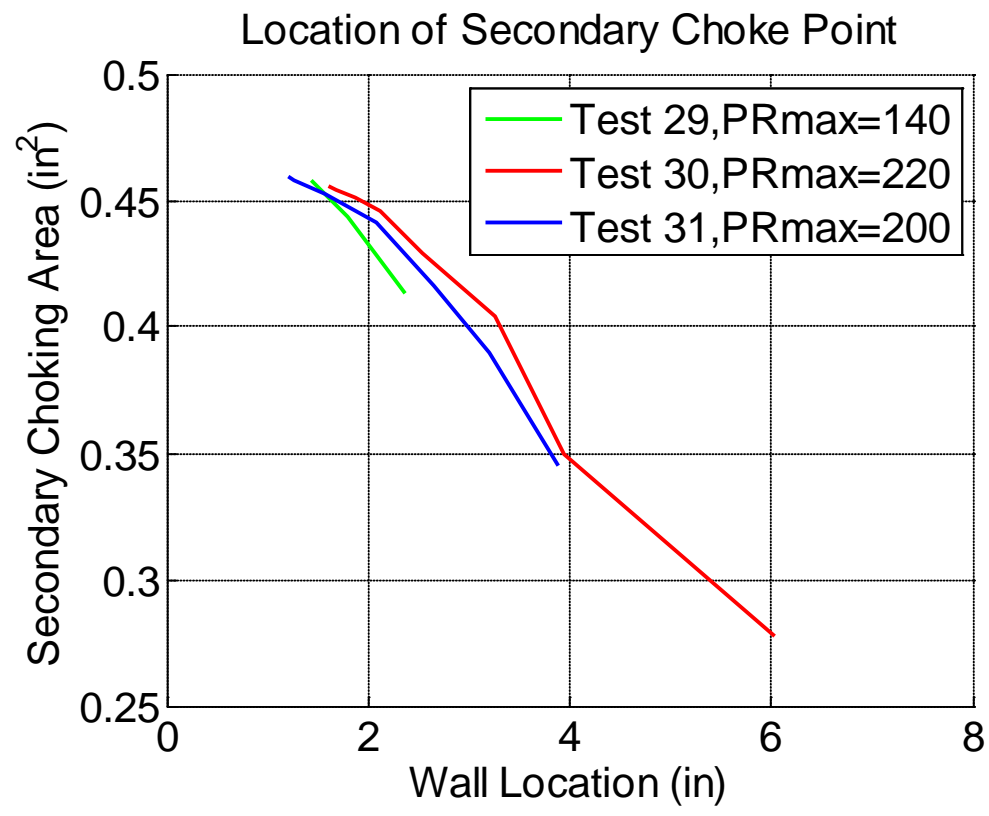

Figure 41: Fabri-choke Location and Size

The Fabri-choke location has a linear relationship to the size of the choking area. The linearity of the function suggests that the secondary flow as a result of the primary plume growth has a steady quality. The highest pressure ratio $(\mathrm{PR}=220)$ occurs when the choke location is 6 inches downstream of the nozzle exit and the aerodynamic choking area is 
$0.25 \mathrm{in}^{2}$. The quasi-steady assumption of the secondary flow can be assumed to be valid and directly dependent on the characteristics of the primary flow.

\subsubsection{Primary Plume Collapse}

In Test 33, pressure transducers along the centerline of the mixing duct were used to calculate the Mach number of the primary flow. The data was used to verify the point in the test when the shock structure of the primary flow breaks down and begins to unchoke. This flow event was observed and noted in Gist's data and can be seen in any test conducted during this test series. In previous tests in this series the only way to observe it was through by observing the rapid static pressure rise that occurred in the wall pressure data at the time of the maximum pressure ratio. By studying the change in pressure along the centerline during a test, the shock structure of the primary flow can be observed. To understand the speed of the flow, the Mach number at each static pressure location was calculated and presented in Figure 42. 


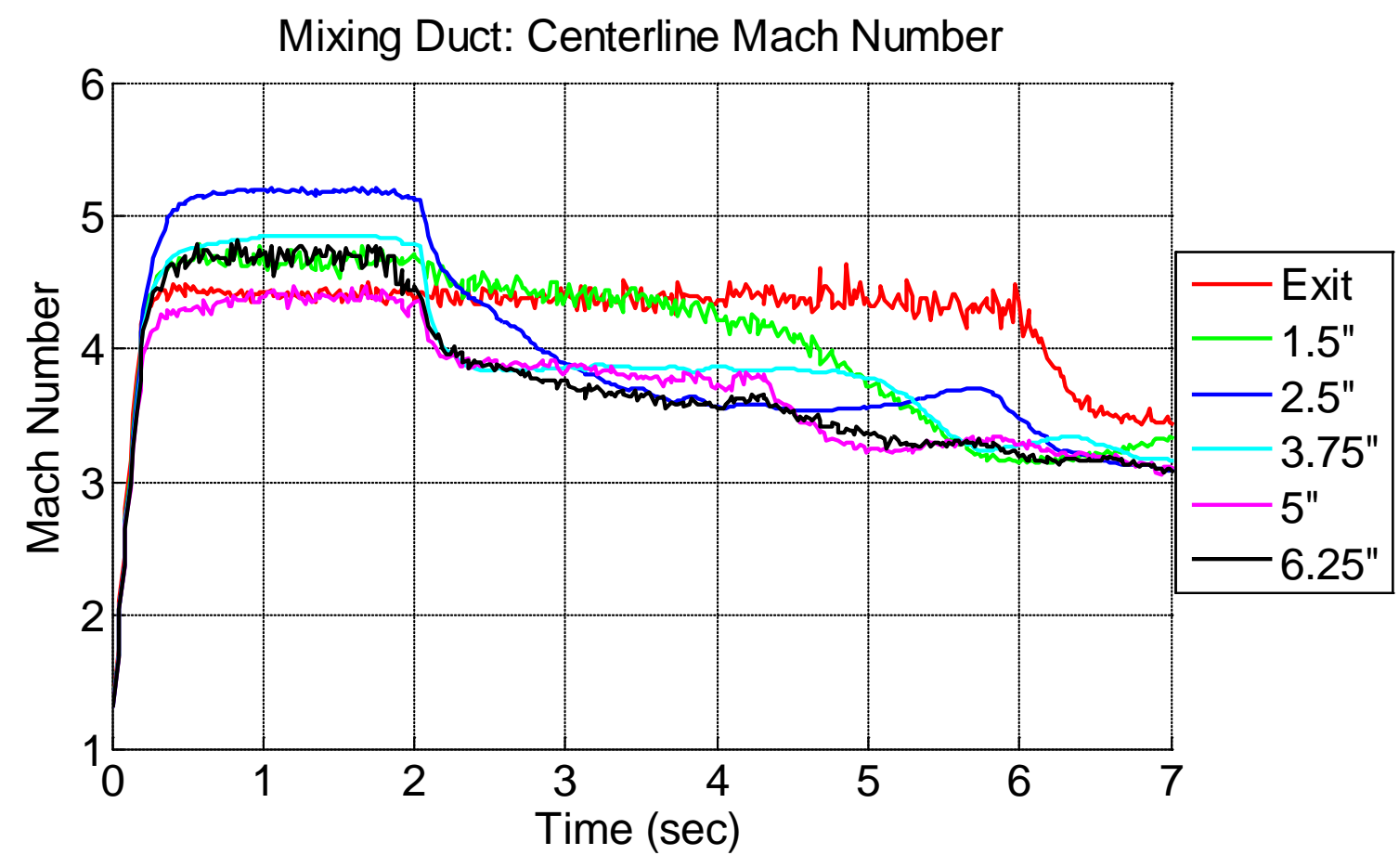

Figure 42: Mixing Duct Centerline Mach number, Test 33

The Mach number was calculated from static pressure at different locations along the primary flow centerline, starting at the exit of the nozzle. The Mach number shown is representative of the shock structure inside the primary plume. The blue, line represents the location 2.5 inches downstream of the nozzle exit.

As shown, the flow quickly becomes established and produces a constant flow structure up until about 2 seconds, the time when the maximum pressure ratio occurs. As the flow structure initially breaks down, it takes another 4 seconds or so for the breakdown to complete. This suggests as the primary plume becomes un-choked at a rate relative to the rate of pressure ratio decrease. Each station downstream of the nozzle exit becomes unstable after the previous concluding in the exit Mach number dropping off at around 6 seconds. The shock moving upstream can be seen clearly in the static pressure data 
recorded along the centerline of the primary plume. The centerline pressure data is shown in Figure 43.

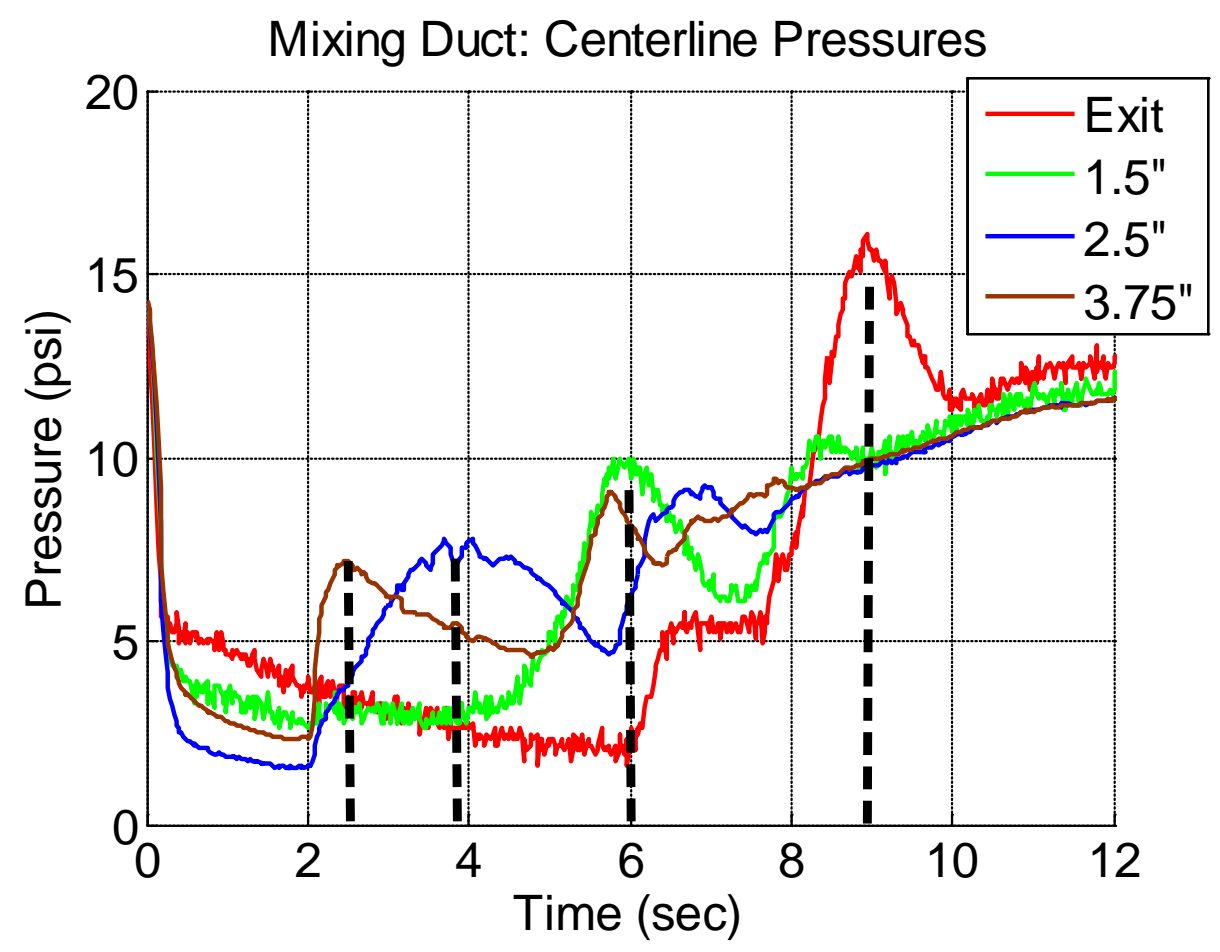

Figure 43: Centerline Static Pressure, Test 33

As the shock moves over a static pressure port, the static pressure will increase instantaneously in an ideal flow, the flow directly after the shock will be composed of expansion waves which reduce the static pressure. These events are seen initially at the breakdown of the flow at 2.5 seconds at the 3.75" mark downstream of the nozzle exit suggesting that the shock moves past this location. The cycle is subsequently verified when a similar event is noticed at 2.5” downstream, 1.5” downstream and then the nozzle exit. Also, as the shock propagates upstream, the time which it takes the shock to move 1" upstream increases as the test progresses. The rate of the shock movement is due to the reduction of chamber pressure and total flow momentum, which influences the rate of pressure ratio decrease. 
This breakdown is also evident in the ability of the plume to entrain from the secondary flow. The almost instantaneous initial breakdown of the shock structure presents itself with a rapid reduction in speed of the secondary flow. The rapid reduction in speed of the secondary flow is seen in the static pressure increase that occurs along the wall as shown in Figure 44.

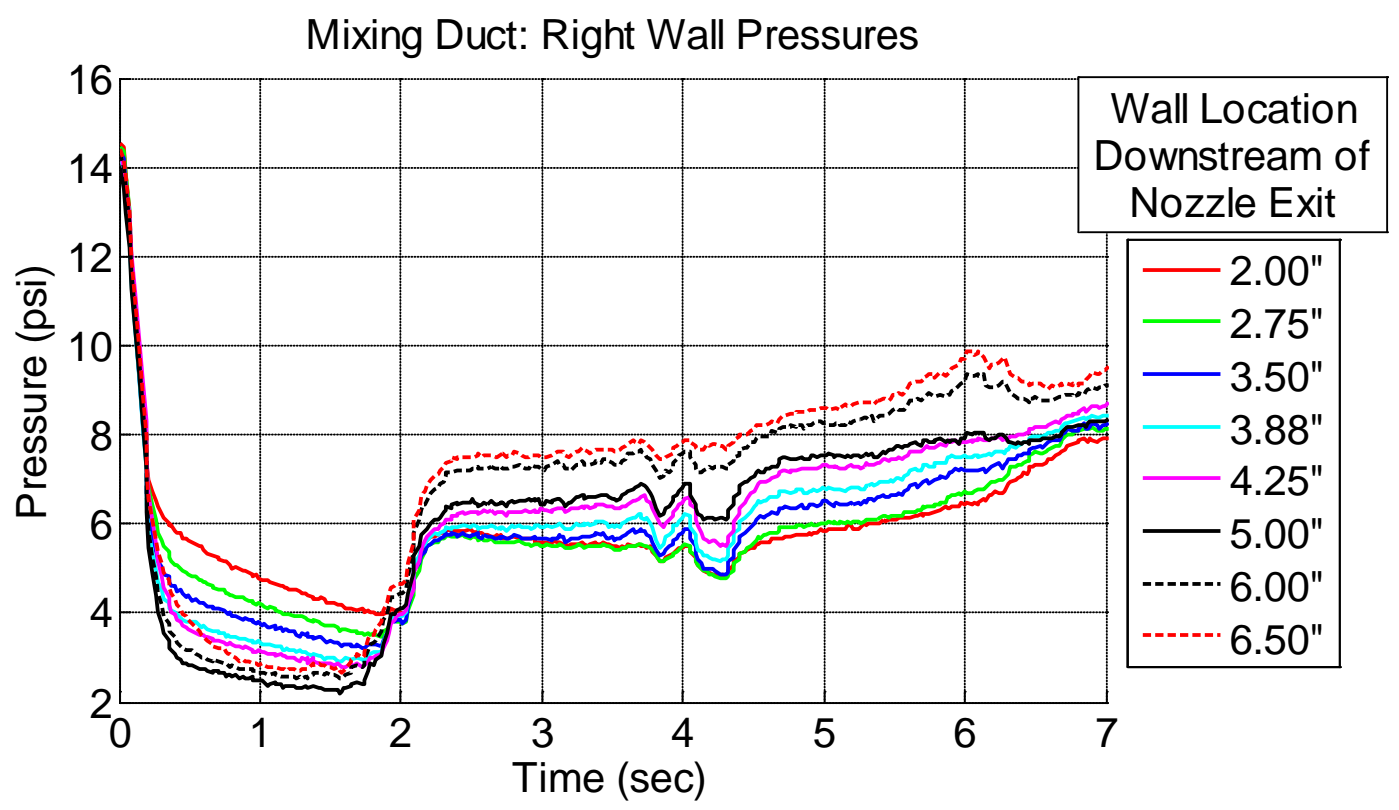

Figure 44: Mixing Duct Wall Static Pressure, Test 33

The static pressures along the right wall of the mixing duct demonstrate a sharp increase in pressure as a result of the shock structure breakdown of the primary flow. An additional factor in the sharp rise in static pressure is the known asymmetry of the flow in the mixing duct. The asymmetry of the flow results in the unsteady and blocked flow observed in the right duct during many of the tests, and is more evident at higher pressure ratios.

After the point of maximum pressure ratio, the inflection point at approximately 2 seconds, the pressure along the wall begins to increase as the secondary flow becomes 
un-choked as the primary plume shock structure begins to break down. After the unchoking of the secondary flow, the static pressures remain relatively constant, from 2 to 4 seconds, showing the blocking of the flow in the right duct or a case where the flow velocity has stopped or becoming significantly low, an event which was not recorded in previous testing.

The relationship between the rapid reduction in secondary flow velocity and primary shock structure breakdown was also observed in previous testing done by Gist. He verified the breakdown of the shock structure initiated the pressure rise along the wall and the asymmetry of the flow through video observation and data interpretation. The pressure data along the wall recorded by Gist is shown in Figure 45 .

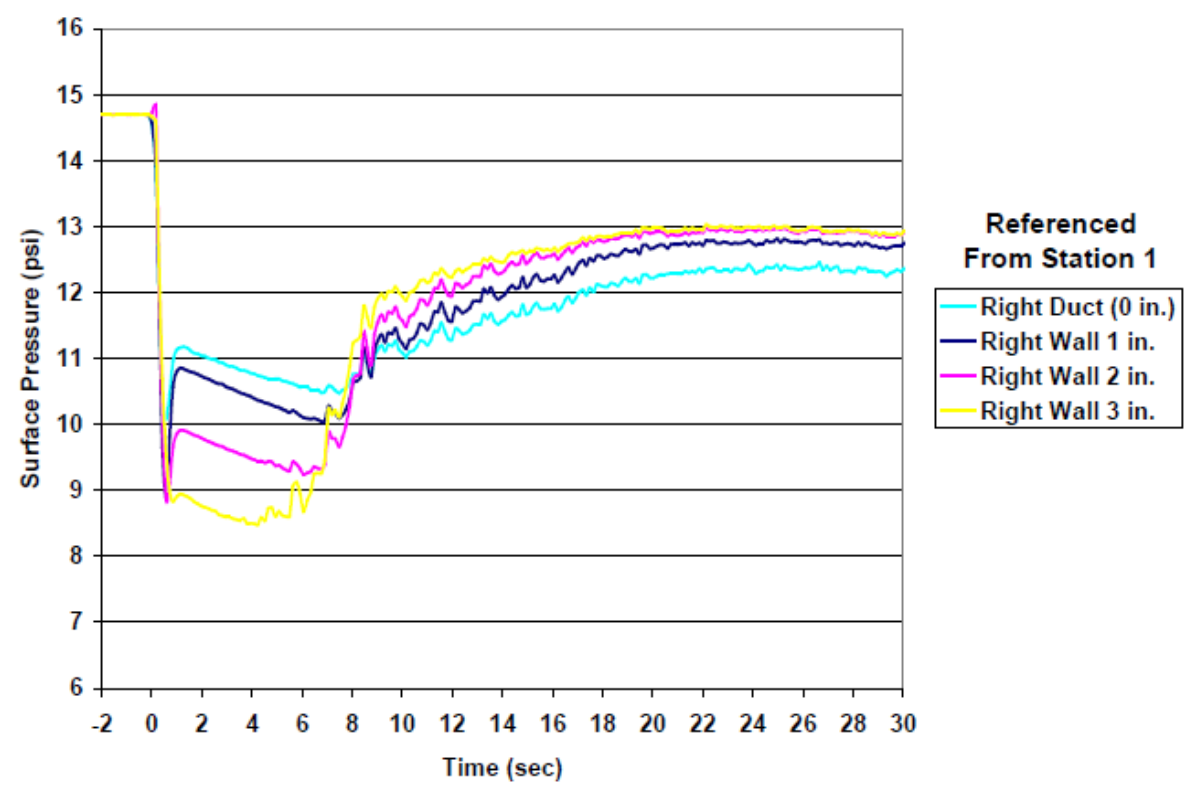

Figure 45: Mixing Duct, Right Wall Static Pressure ${ }^{4}$

The un-choking of the secondary flow along the right duct was observed in Gist's tests as well, shown at about 6 seconds. While the pressure ratio is high, the secondary flow experiences a favorable pressure gradient as it moves downstream toward the Fabri- 
choke location. The secondary un-choke occurs at a pressure ratio of 95 in Gist's test, at the 3 inch location ${ }^{4}$. Turbulent flow of the right duct becomes evident after the un-choke process. By referencing Gist's data, we can show how based on the PR, the location of the aerodynamic throat of the secondary flow changes. In Figure 44, the un-choke occurs at a pressure ratio of approximately 160 .

Based on the nozzle geometry and the chamber pressure, the primary flow has enough momentum to maintain a constant shock structure for a time dependent on the primary flow pressure rate of decay. During this time, as the primary flow under-expands it causes the secondary flow in the left and right ducts to choke in the mixing duct. The expansion of the primary plume causes an aerodynamic throat to form in the mixing duct for the secondary flow of each duct. Several factors affect the breakdown of the shock structure in the primary flow, the loss in primary flow momentum, the low stagnation pressure of the secondary flow and peak pressure ratio. As the primary plume decreases in size, the shock structure begins to break down and propagate upstream, causing the aerodynamic throat location of secondary flow to increase in area and un-choke. As the secondary flow becomes un-choked, the flow decreases speed rapidly, which is shown by the rapid rise in static pressure. By examining pressures recorded along the wall and along the centerline, the relative size and characteristics of each flow can be examined. The expansion and breakdown of the primary plume is represented in Figure 46. 


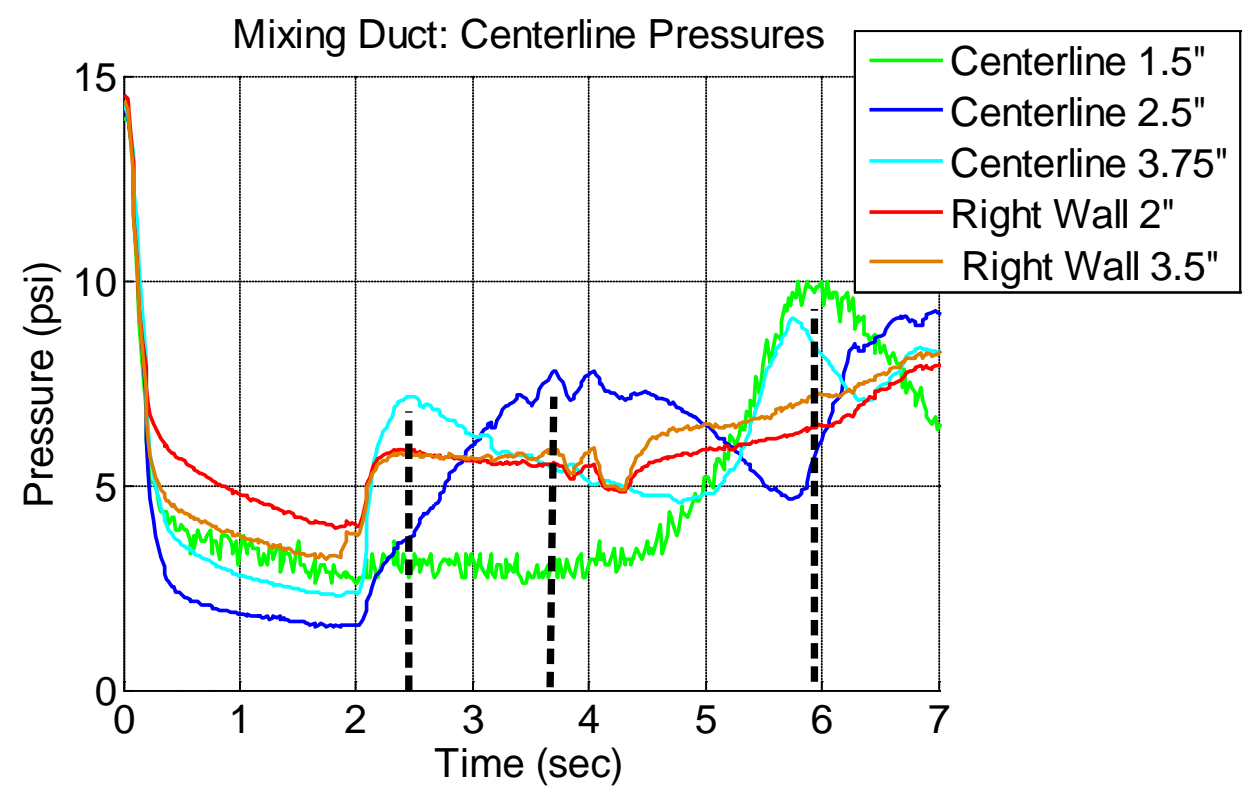

Figure 46: Plume expansion and breakdown, Test 33

After the initial transient start up, the flow achieves quasi-steady flow (0.4 to 2 seconds). If a constant pressure plume boundary condition is applied to the flow in the mixing duct, it can be assumed that the pressure along the wall is equal to the pressure at the primary plume boundary. The peak pressure ratio of this test was lower than the highest pressure ratio tests; viscous effects at the plume boundary are not as present. The pressure along the boundary (Wall 2” and Wall 3.5”) is larger than the centerline of the plume suggesting an under-expanded plume. The breakdown of the primary flow (2 seconds) also coincides with the un-choke of the flow along the wall, causing a period of constant pressure flow along the wall (2 seconds to 3.8 seconds) as the primary un-choke propagates upstream, this period onward suggests an instability which can be seen consistently across all of the tests. 


\subsubsection{Unsteadiness and Asymmetry}

The plume of the primary flow expands up to its maximum point at the maximum pressure ratio of a test (at $\mathrm{t}=2$ seconds in Figure 47). The data recorded after the maximum pressure ratio produces interesting flow phenomena in the secondary flow because the momentum of the primary flow is sufficiently low enough such that it can no longer entrain mass from the plenum. Secondary mass flow continues for another 2.5 seconds ( $t=2$ to 4.5 seconds) due to the size of the recirculation zone at the lip of the nozzle. This recirculation zone produces an induced mass flow transfer between the two flows ${ }^{8}$. At this point ( $t=4.5$ seconds) the pressure in the plenum begins to increase but the flow in the right duct becomes blocked due to an asymmetry of the flow. The blockage of the right duct causes a dramatic reduction in velocity of the flow in the right duct that is not seen in the left duct, as shown in Figure 47.

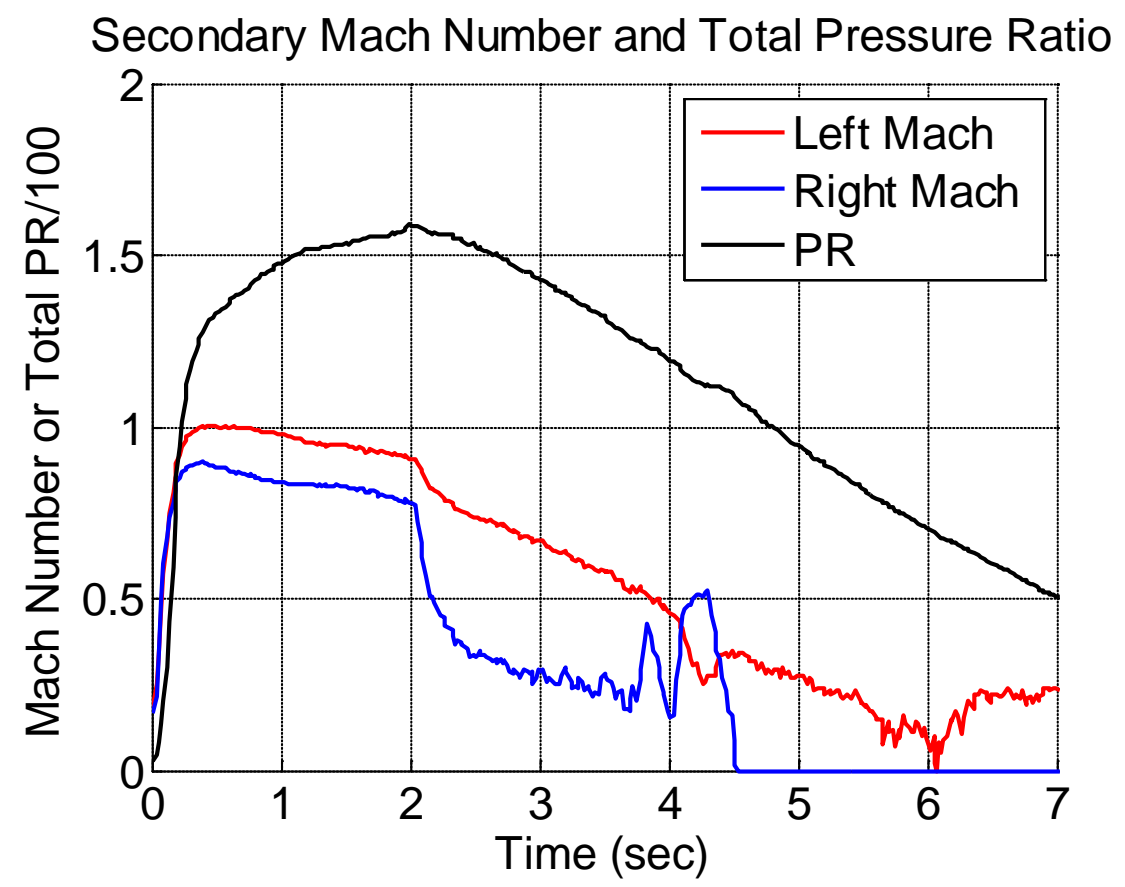

Figure 47: Test 33, Secondary Mach number and Total Pressure Ratio 
The unsteadiness is also associated with the breakdown of the primary flow after the point of maximum expansion (maximum pressure ratio, 2 seconds). A sharp reduction in the Mach number of the right duct occurs when the primary flow begins to un-choke. The secondary flow begins an oscillatory behavior and then the secondary flow becomes blocked in the right duct. The unsteady oscillatory region can be assumed to be attributed to the formation of a recirculation zone in the region where the two flows begin to interact at the exit of the nozzle. As the oscillations grow in intensity, the secondary flow of the right duct becomes completely blocked off. The blocking occurs due to the asymmetry of the flow. The unsteady region of the test is where the asymmetry of the flow becomes the most obvious and exaggerated.

Despite the large pressure ratio at points in time after the maximum pressure ratio has been achieved, the data calculations can no longer assumed to be valid as turbulence in the flow affects the validity of the isentropic flow assumption.

As shown in Figure 48, the pressure of the flow along the right wall of the mixing duct becomes un-choked and remains relatively constant with the breakdown of the primary flow. 


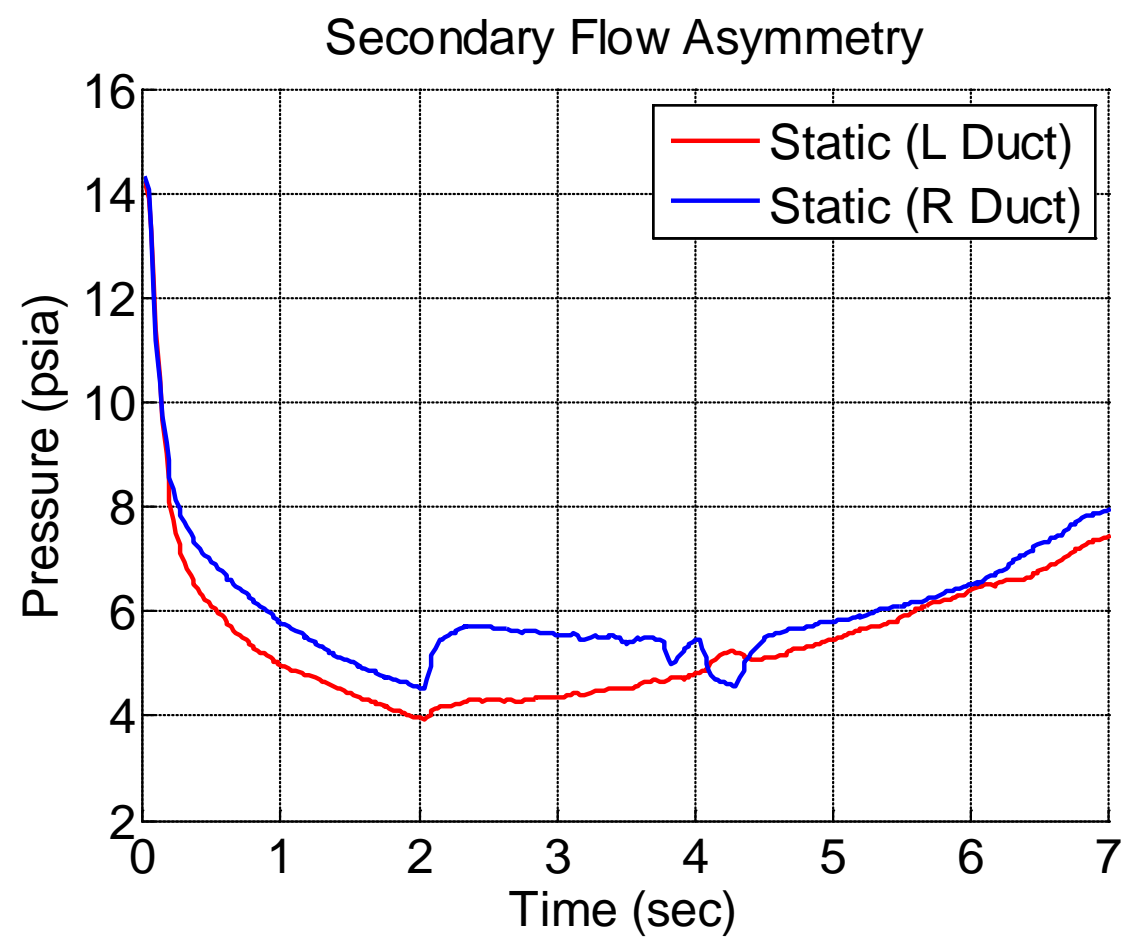

Figure 48: Test 33, Flow Asymmetry

The pressure of the primary flow is still high enough to retain the pressure in the right duct along with the asymmetry of the primary plume, which also allows pressure to bleed into the left duct to pressurize the plenum. If the ducts were perfectly symmetrical, we would see the pressure in both ducts increase at the same time and at the same rate. But the asymmetry causes an uneven pressurization of the plenum as the primary plume breaks down. The asymmetry can be seen at 2 seconds (maximum pressure ratio) when the static pressure of the right duct spikes and remains relatively constant for another 2 seconds. The asymmetry is further shown in the oscillation of the left and right duct pressures at roughly 4.25 seconds. This unsteadiness in other tests produces cases where the secondary flow of the right duct appears to become completely blocked.

The blocking of the right secondary duct can also be seen as a function of the Mach number along the wall. As the primary plume achieves maximum expansion, the flow un- 
choke process begins at the most downstream location where the choke point is located. Figure 49 shows the Mach number along the wall for the entire transient region.

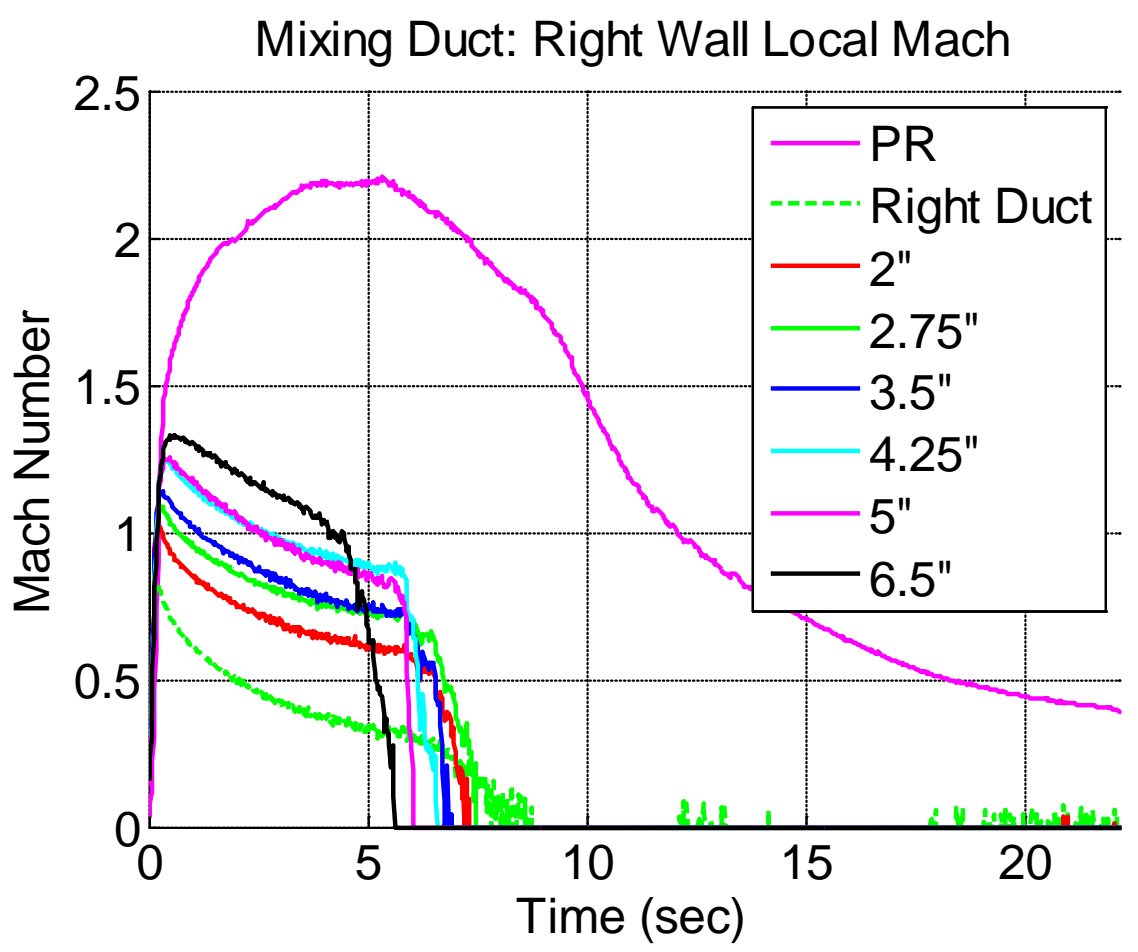

Figure 49: Test 30, Mixing Duct Right Wall Mach

When the flow achieves the maximum pressure ratio at approximately 4.8 seconds, the un-choke process begins. The beginning of the un-choke can be seen at pressure port 6.5” where the Mach number is 1 (choke point) and then suddenly decreases. After the initial un-choke process, we can see the unsteadiness of the un-choke process propagate upstream as the Mach number at each point drops to zero, resulting in a static flow condition where entrainment has ceased. The flow in the mixing duct along the right wall becomes completely blocked as the Mach number in the right secondary duct becomes zero at $\sim 8$ seconds.

The unsteadiness that propagates upstream is also shown in Figure 42 with the primary shock structure break down as pressure ratio decreases. As the shock structure of the 
primary plume degrades, the pressure increase in the secondary flows is caused by pressure bleed in through the boundary layer ${ }^{21}$. The asymmetry of the geometry causes flow in the right duct to become "blocked" or in this case blocks pressurization of the plenum. The blocking that occurs becomes more apparent as the pressure ratio is higher as shown in Tests 30 and 31 over Test 33. The flow is blocked because the entrainment of the flow in the right duct has completely ceased, as shown in Figure 50.

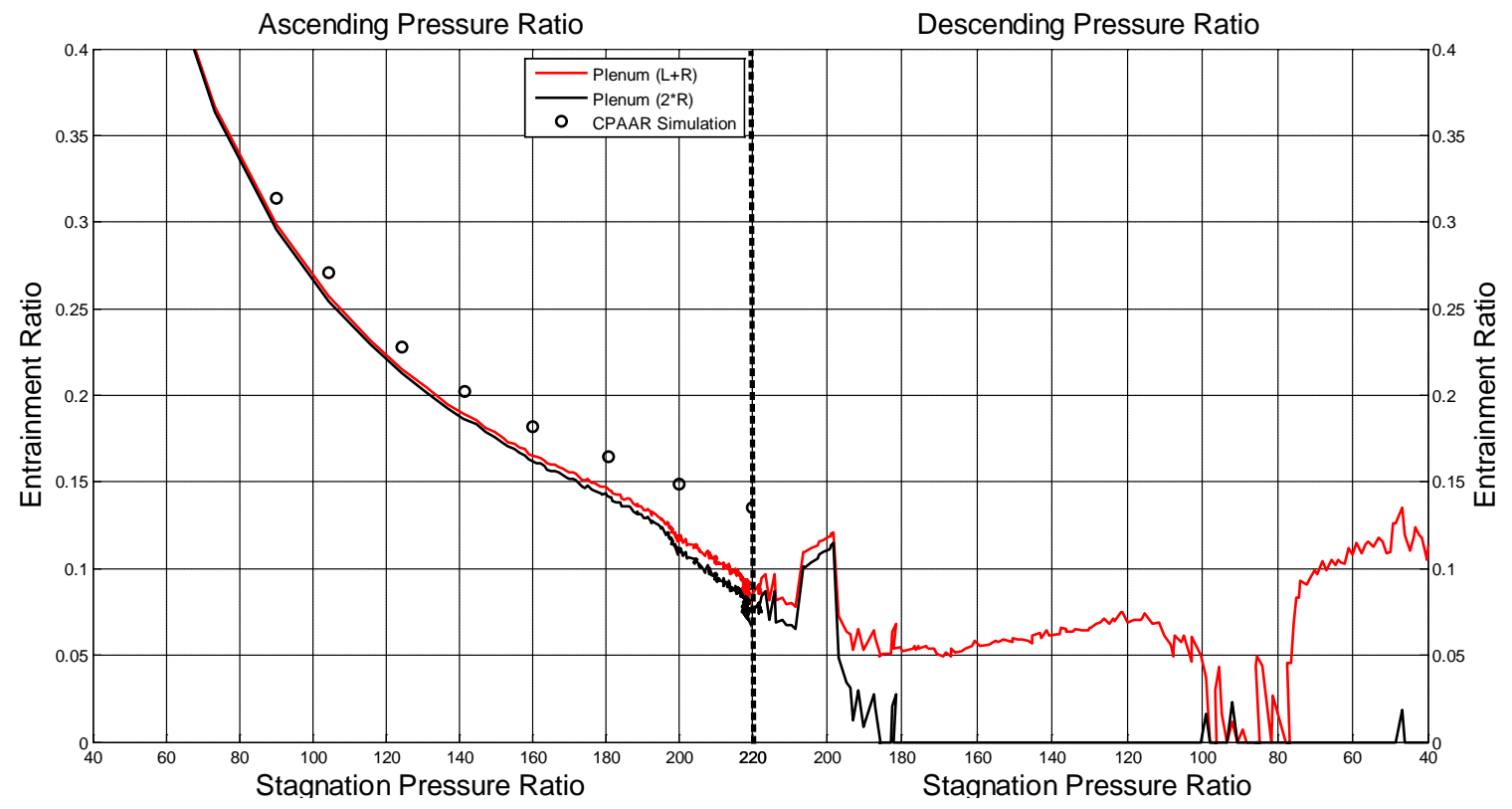

Figure 50: Test 30, Ejector Performance and Right Duct Block

The red line represented the entrainment calculated using the mass flow rate of both ducts, while the black line represents the calculation using only the right duct (multiplied by 2 to represent a total flow). The left plot shows the relationship as pressure ratio is increasing from test start up to the maximum pressure ratio, and the right plot shows the relationship as the shock structure of the primary flow is breaking down. In Figure 50, the left plot would represent from 0 to 5 seconds, and the right plot would represent from 5 seconds on. The unsteadiness occurs almost instantaneously after the primary flow 
becomes un-choked. The flow along the right duct becomes completely blocked after the unsteadiness appears and the asymmetry becomes much more evident as flow is still present in the left duct. This asymmetry was a noted feature of the CPAAR from previous tests and research ${ }^{4,21}$. At high pressure ratios the asymmetry causes a blocked condition.

\subsection{Plenum and Secondary Duct Results}

The primary improvement in the test hardware for this research lies with the addition of the plenum and the transient condition added to the flow. To understand the affect of the plenum on the data produced by the test series, the losses in the secondary ducting are presented below, along with the previously studied asymmetry of the flow in the mixing

duct ${ }^{4}$. The transient analysis of the flow is also presented. The flow is considered Quasisteady in the transient region of the test, and two subsections of the transient region are defined and analyzed.

\subsubsection{Secondary Flow Measurement Differences}

The final design of the secondary inlet system was done to mitigate the losses associated with the previous iterations. While the final constant area secondary inlet system produced a more laminar and steady flow and was shorter than the previous designs, the total length of the secondary inlet system was increased by 7 inches from the original secondary inlet system used in the Gist and Foster research. The additional length resulted in a pressure loss due to friction and was observed during testing. Based on the geometry of the secondary ducts, an inviscid flow, with no leaks or discontinuities the expected losses along the inlet system is less than $5 \%$ of stagnation pressure loss based on losses associated with flow in a square duct. The stagnation pressure recorded at the 
pitot probe was consistently lower during a test than the pressure recorded upstream in the plenum as shown in the Figure 51.

Inlet Losses

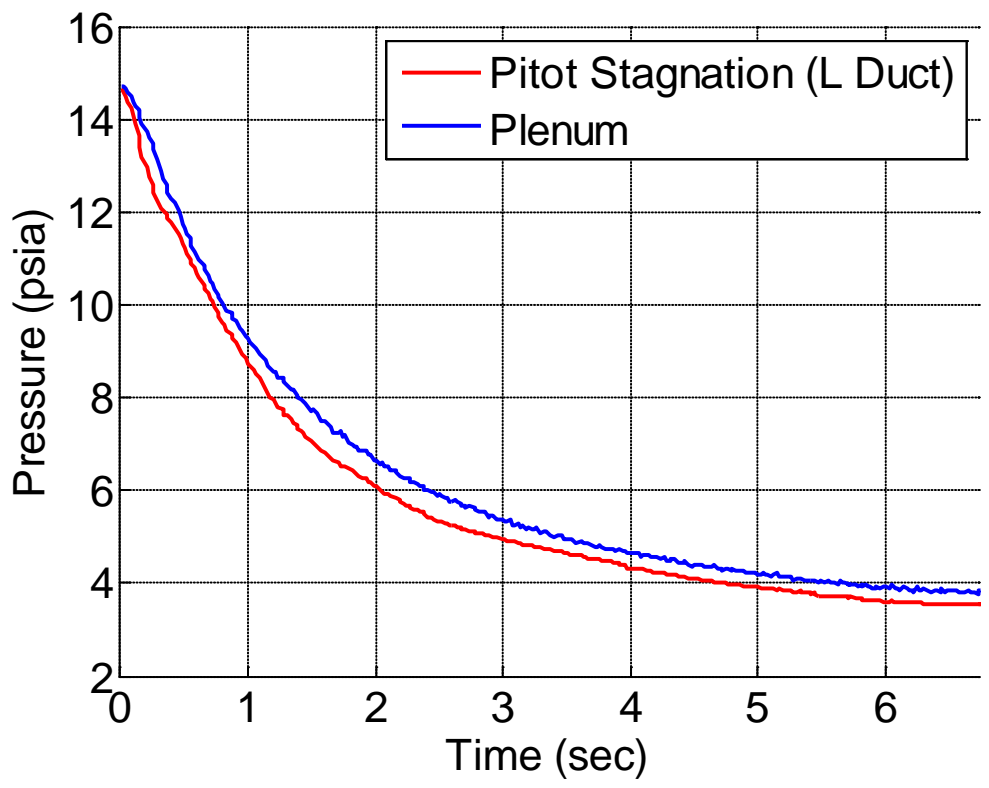

Figure 51: Test 31, Secondary Inlet, Left Duct Stagnation Pressure Loss

The pressure recorded in the plenum is consistently higher at any point during the first 15 seconds of Test 31 . The maximum difference during this time period was a 0.95 psi lower pressure recorded at 3 seconds at the stagnation point of the pitot probe in the left duct. This lower pressure is associated with the losses due to friction and the geometry of the secondary ducts. Across the formal testing, pressure losses as large as $9 \%$ were observed between the pitot calculation and plenum calculation. The actual losses in the duct were about $4 \%$ larger than the expected losses, possibly due to leaks in the inlet and manifold.

The losses result in different calculated pressure ratios, Mach numbers and mass flow rates relative to the stagnation pressure used in the calculation. For the overall process, the losses observed in the tests were considered negligible and the pressure in the plenum 
was used for left and right duct flow calculations to maintain a level of symmetry in the data.

The stagnation pressure recorded at the pitot probe in the left duct was not assumed to be similar to the stagnation pressure in the right duct at a similar location due to an asymmetry observed in the flow. The results section from here on will specify the location of the stagnation pressure data used in the calculation for any value based on secondary stagnation pressure.

\subsubsection{Flow Asymmetry}

The availability of instrumentation dictated the configuration of the instrumentation as mentioned previously. The most significant issue with this fact was associated to the measurement of the secondary flow data. A pitot probe was only placed in the left duct which did not allow for the direct measurement of the stagnation pressure at a location close to the mixing duct of the right duct. Static ports were available in both ducts and produced data which replicated an asymmetry observed in previous testing done by Gist ${ }^{4}$ and Foster ${ }^{9}$. Figure 52 plots the static pressure data of the left and right secondary ducts during Test 31. 


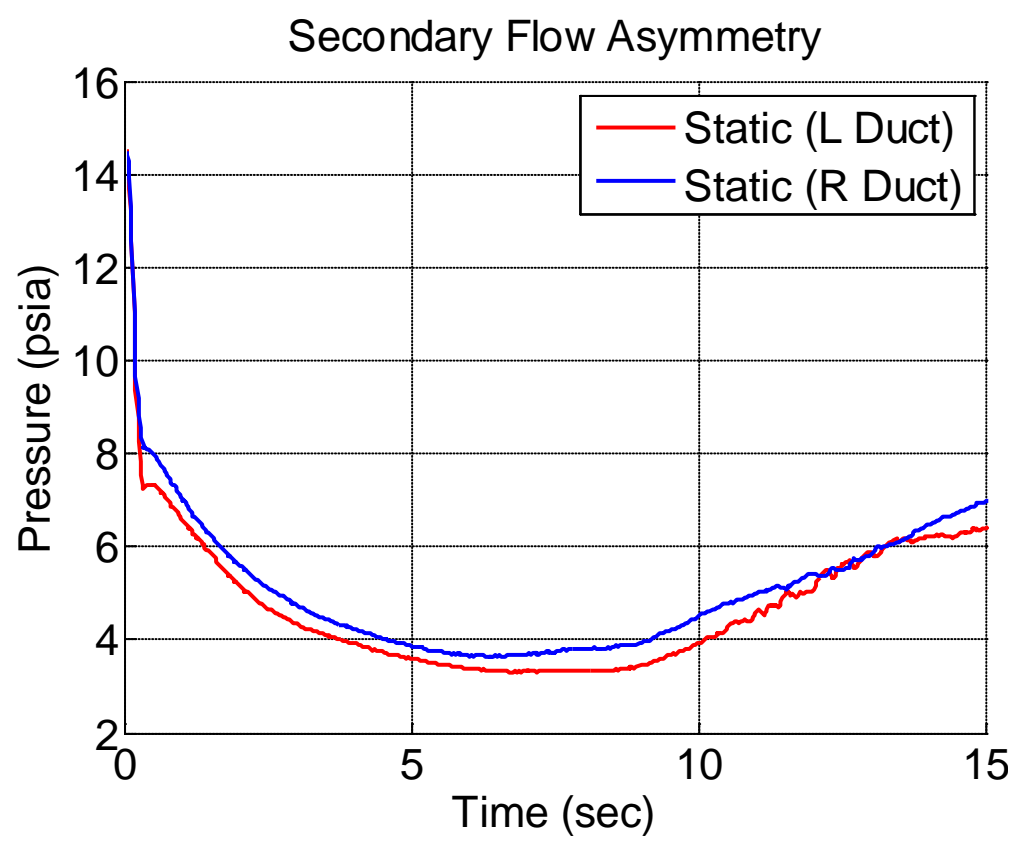

Figure 52: Test 31, Secondary Duct Asymmetry

The static pressure of the left duct is consistently lower during the test, which suggests the flow is of a higher velocity in the secondary duct than the right duct. This asymmetry was suggested to be as a result of the structure of the primary flow ${ }^{4}$. Again, although the data suggests a $9 \%$ difference in the recorded pressure, the difference was worth noting and taking into account during the analysis.

Due to the difference in the flow velocities of both ducts and the losses associated with the inlet, only a specific set of data was used in reference to the analysis of the mixing duct. During calculations of flow in the mixing duct, stagnation pressure of the secondary flow was considered equal to the pressure recorded in the plenum.

\subsubsection{Plenum Quasi-Steady Assumption}

The analysis of the flow data is dependent on the assumptions for the equations used. Addy has suggested for supersonic air-to-air ejector transient analysis problems, the 
application of a quasi-steady assumption is acceptable for flows fields with similar conditions as the $\mathrm{CPAAR}^{7}$. To study the steady state assumption it was critical to determine a portion of each test which could be compared against each other as the section of the test to be used for performance analysis.

The CPAAR is operating in a consistent transient condition, and based on previous findings ${ }^{7.8}$, we know that the majority of the transient region can be assumed quasi-steady as long as that region is the approximately greater than $98 \%$ of the total transient region. To determine if the startup transient is a sufficiently low percentage of the total transient region we can look when entrainment starts and becomes established. By observing the starting transient of the entrainment ratio as a function of the characteristic time of the transient region we can verify if the quasi-steady assumption of the flow is valid as shown in Figure 53.

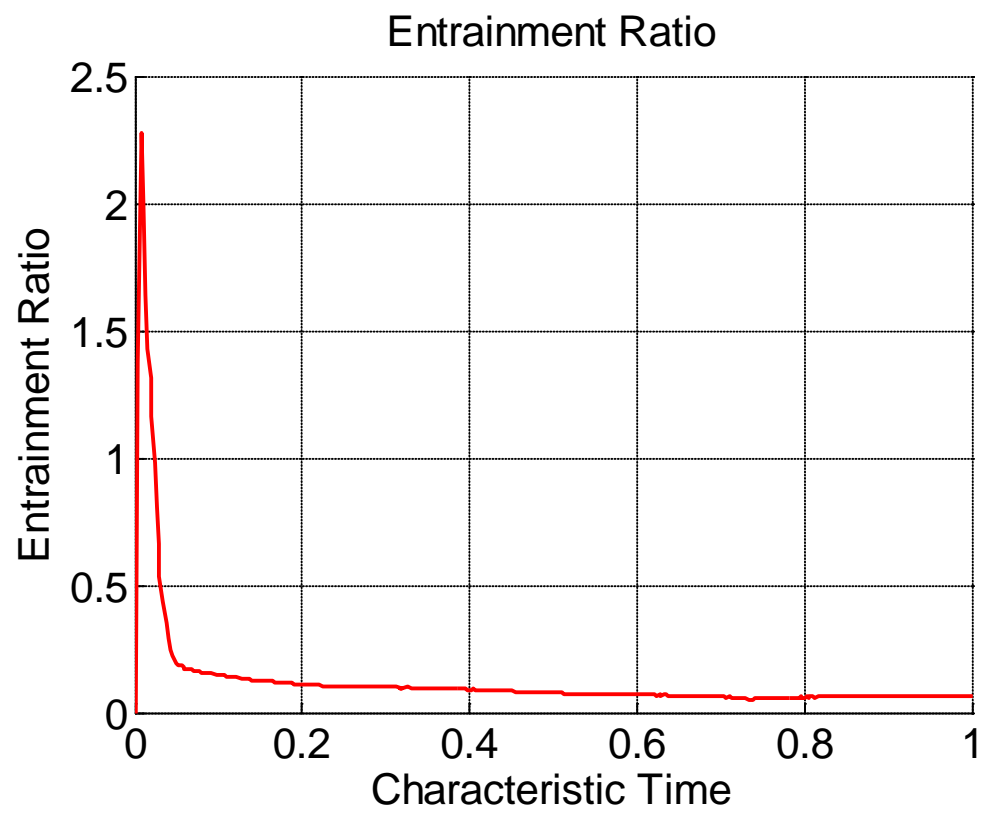

Figure 53: Test 31 Transient Region 
If compared to Figure 12, the profile the flow is almost identical, with a small discrepancy at the end of the characteristic time when entrainment ratio is equal to zero. The difference lies in the fact that during this series of testing we did not achieve a fully Fabri-blocked case. In Table 1 below we can see the values of the starting transient regime as a percentage of the total transient regime. The total transient time, is the time as defined previously, from initial pressurization of the primary chamber, to the minimum pressure achieved in the secondary flow is represented in Table 3.

Table 3: Test Transient

\begin{tabular}{|c|c|c|}
\hline Test & Total Transient (sec) & Startup Transient (\%) \\
\hline 29 & 4.11 & $2.7 \%$ \\
\hline 30 & 7.39 & $1.1 \%$ \\
\hline 31 & 6.79 & $1.8 \%$ \\
\hline 33 & 4.06 & $2.4 \%$ \\
\hline
\end{tabular}

Tests 30 and 31 fall within the 1-2\%, while the others are below 3\%. We can assume all of the tests are operating in a quasi-steady state and should be comparable to a steady state test, but do not explicitly satisfy conditions for a steady state flow. The transient region will incorporate the pressurization and discharge of the primary chamber, and obtain the minimum secondary flow stagnation pressure. The entire range of the transient region is shown in Figure 54. 


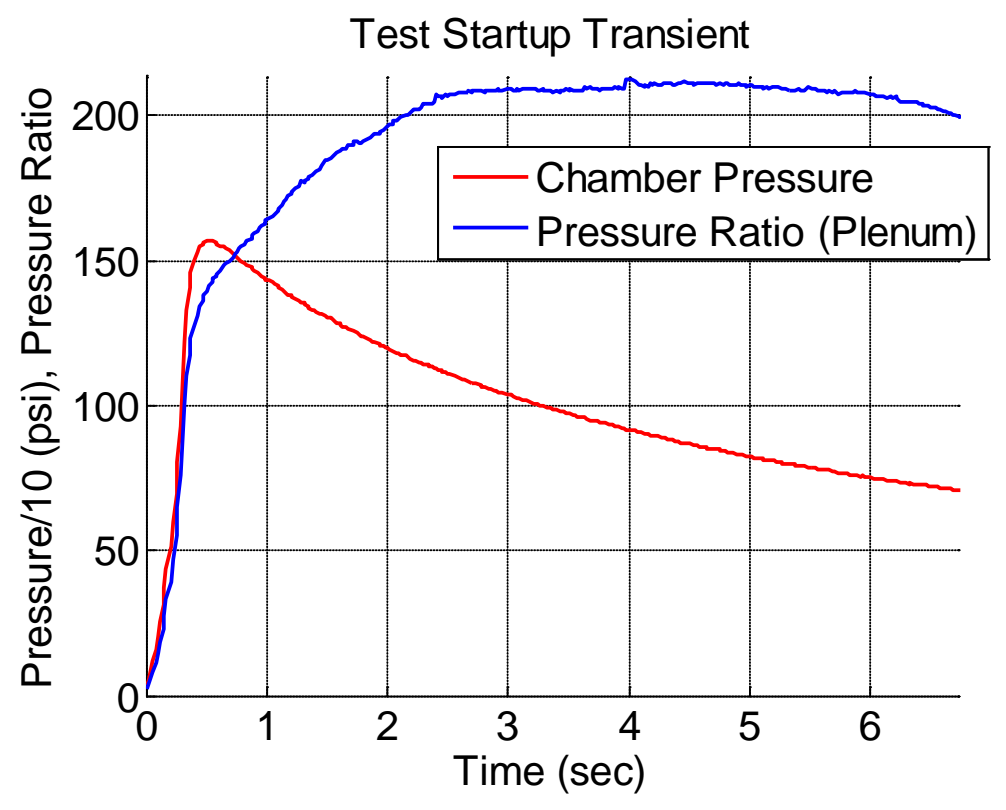

Figure 54: Test 31, Total Transient Region

The total transient region incorporates the rapid pressurization of the primary chamber and subsequent discharge. The pressure ratio peaks at approximately 2.5 seconds, stays constant for $\sim 3.5$ seconds and begins to decrease during this transient region, suggesting that the quasi-steady assumption is applicable for the majority of the data recorded during a test despite the continuous transient properties of both flows.

Because of limits of the hardware and ability to sustain steady and symmetric flow in the primary, it is necessary to define regions in the quasi-steady transient region for further analysis. The ability of the primary flow to entrain begins to fade after the maximum pressure ratio is achieved as the primary chamber continues to discharge. Therefore we can define a new "Working Transient" region to end at the point of maximum pressure ratio. Thus the working transient region will be defined as quasi-steady also. The data produced after the maximum pressure ratio is achieved although, still technically 
qualified as quasi-steady based on characteristics of the secondary flow, now has a third order transient property based on the reduction of momentum of the primary flow.

Figure 55 represents the working transient region for Test 31 as defined as ending at the maximum pressure ratio.

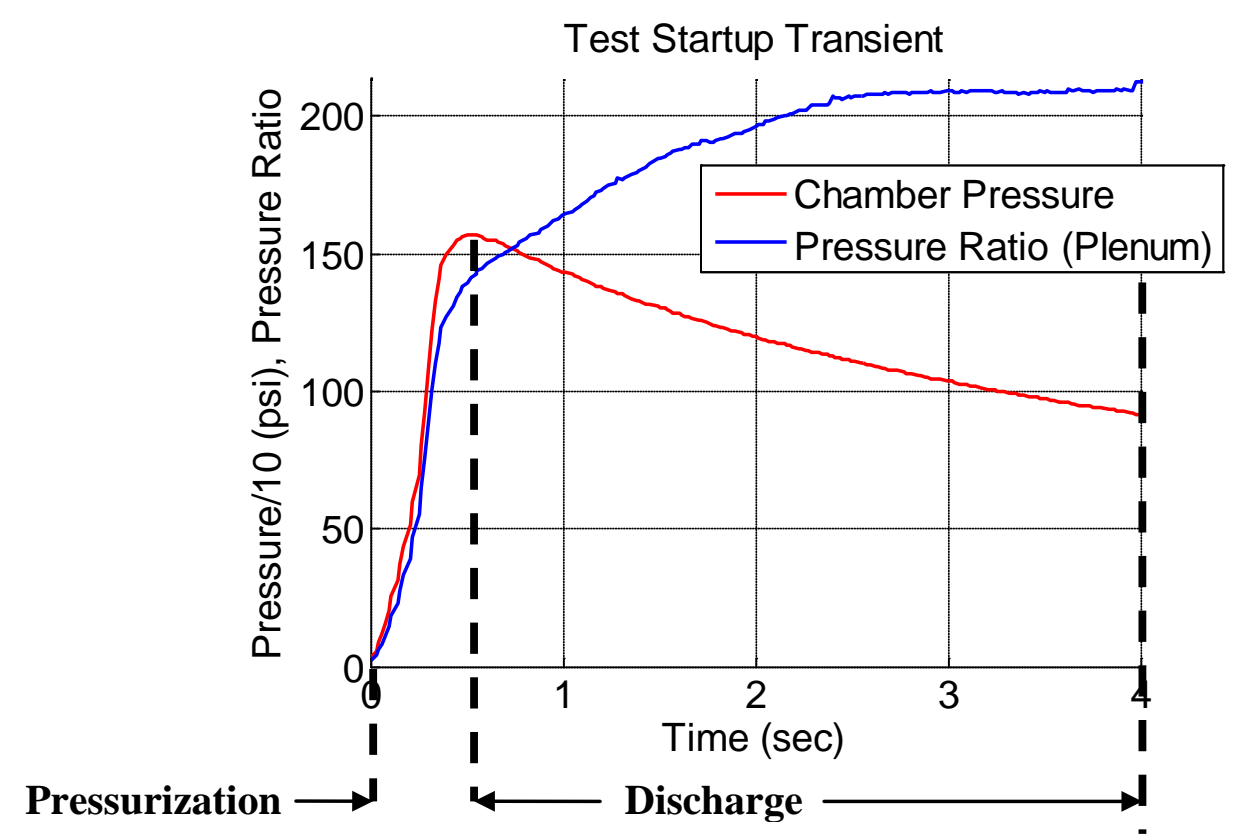

Figure 55: Test 31, Maximum Pressure Ratio during Working Transient Region

The working transient region is time from the actuation of the primary valve to the time when maximum pressure ratio was achieved. A significant change in the rate of pressure ratio increase is observed at the inflection point of the maximum primary chamber pressure at approximately 0.5 seconds. This time slice of each test was observed and divided into two sections; the primary chamber pressurization transient and, the chamber discharge transient. Table 4 shows the relative percentage of the time slice of each working transient region, and the associated pressurization and discharge transient percentages. 
Table 4: Test Transient

\begin{tabular}{|c|c|c|c|}
\hline Test & Time Slice (sec) & Pressurization Transient (\%) & Discharge Transient (\%) \\
\hline 29 & 2.45 & $14.3 \%$ & $85.7 \%$ \\
\hline 30 & 5.34 & $8.3 \%$ & $91.7 \%$ \\
\hline 31 & 3.98 & $13.1 \%$ & $86.1 \%$ \\
\hline 33 & 1.99 & $19.6 \%$ & $80.4 \%$ \\
\hline
\end{tabular}

For low pressurization transient percentages, we can suggest that the rapid pressurization of the primary flow produces a primary plume which establishes itself in the mixing duct quickly and could establish a Fabri-choking case in a relatively short period of time. Depending on the startup transient percentage, a saturated flow condition may only be inferred and not directly measured due to recording rate of the data. By observing the Mach number in the secondary duct, the saturated case should be visible as producing Mach 1 flow in the secondary duct. The Mach number in the secondary ducts during the working transient region is presented in Figure 56.

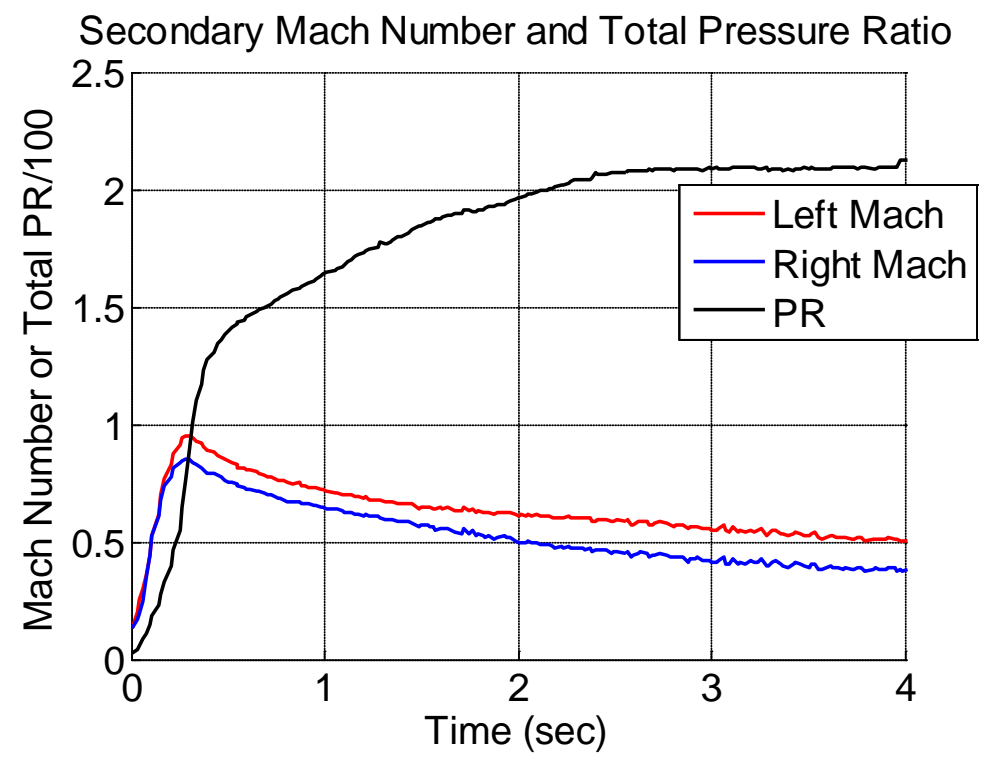

Figure 56: Test 31, Secondary Mach number and Total Pressure Ratio 
The Mach number of the secondary flow in the left duct shows the peak Mach number to be just below Mach 1, suggesting a flow that increases in speed to its maximum point and inflects in correspondence with the pressurization and discharge transient inflection point. The flow does not achieve a saturated flow condition, where the flow achieves Mach 1 in the secondary duct. The secondary flow does not choke at the duct most likely as a result of the quick transient start up process of the secondary and primary flows. The visualization of this can be seen by the rate of increase of the Pressure Ratio. The Mach number inflection point suggests that the establishment of the secondary flow is directly related to the transient pressurization region of the primary flow. Figure 57 is of Test 33, which has a higher percentage of transient pressurization.

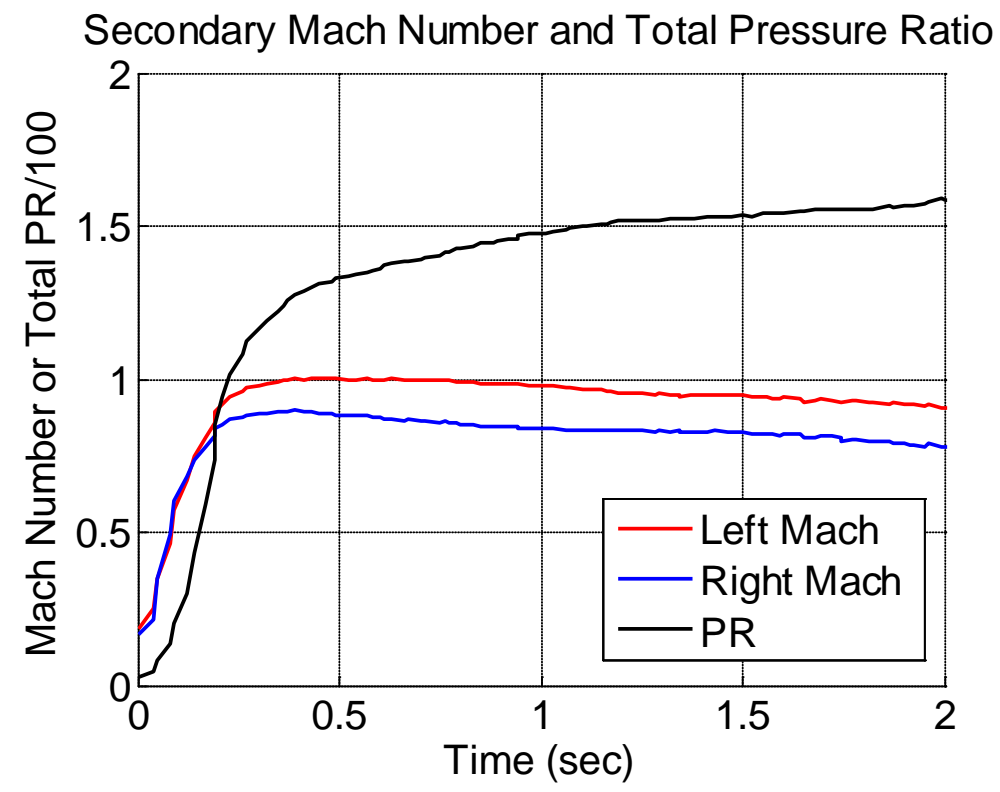

Figure 57: Test 33, Secondary Mach number and Total Pressure Ratio

Test 33 had a higher percentage of the pressurization transient than Test 31, and was able to obtain a saturated case in the left duct. This suggests that the rate at which the primary flow becomes established is related to the establishment of the secondary flow saturated case. The secondary flow does not become fully established until after the transient 
inflection point. After this, the primary flows shock structure is fully formed and allows for observation of the transient flow-field and Pressure Ratio increases to its maximum value.

The Mach number at the end of the secondary duct would be 1 for the saturated flow condition, but that is not observed by the data for Test 31 but is for Test 33 which had a higher pressurization transient percentage. The saturated flow does not become established due to the quick transient startup of the CPAAR in tests with low pressurization transient percentages. The flow-field instead, establishes to some form of Fabri-choking dependent on what the pressure ratio is when the transient inflection point occurs.

\section{Comparison}

Entrainment Ratio is independent of Pressure Ratio and highly influenced by the primary mass flow rate. During the course of formal testing, the highest peak pressure ratio achieved was 220 and the lowest peak was 134 . The tests produced data which performed identically in terms of the performance of the ejector. The repeatable performance of the CPAAR shows the flow-field behaves consistently across several

different tests and allows for cross analysis of the data. Figure 58 is a performance plot of the formal testing of the system. 


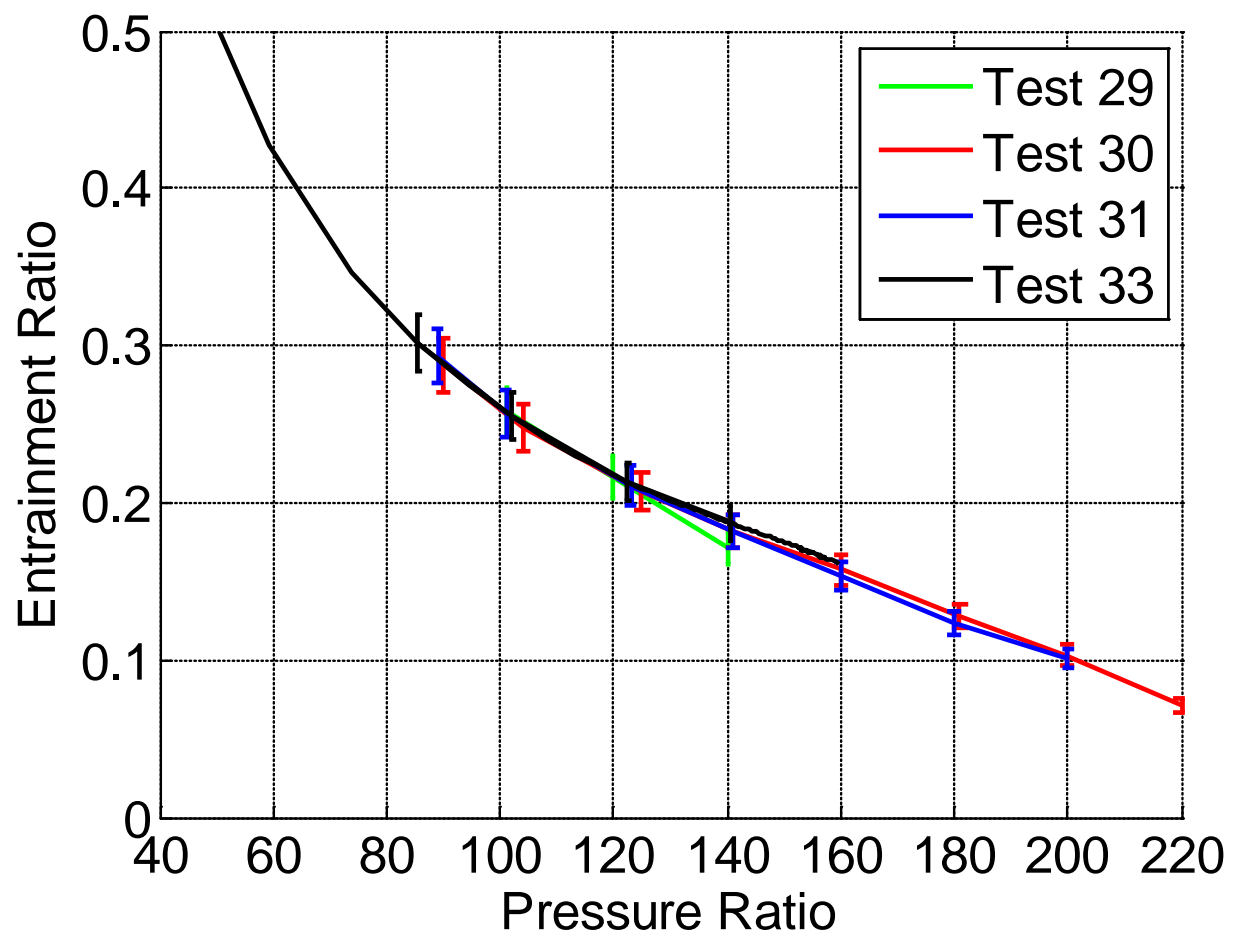

Figure 58: Formal Testing Ejector Performance

All of the tests replicate performance for similar pressure ratios up until the maximum performance of the test. At this time an uncharacteristic reduction in entrainment occurs during Test 29, due to the unsteady nature of the primary plume degradation and its affect on pressure measurements at the maximum pressure ratio inflection point.

Gist observed the instantaneous pressure increase along the wall of the duct during testing, the un-choke process of the primary flow. In this test series that behavior has been observed at pressure ratios similar to those tests by Gist. At very high pressure ratios, such as those above 180, it has been observed that the instantaneous pressure increase of the plume shock breakdown becomes a gradual process. The change in pressure along the wall is relative to the rate of change of the pressure ratio, which at during the test is a function of the decrease in primary plume degradation and increasing secondary flow pressure. 


\subsection{CPSE Simulation}

Based on the data collected, the total momentum of the primary flow along the plume boundary was not sufficient to expand and block off the secondary flow for the tests conducted during this test series. But the simulation was able to calculate some values based on flow conditions recorded during several tests. There were several issues related to the calculation of the entrainment ratio by the code, one such issue was the emergence of discontinuities in the plume Mach number.

The following results were obtained from the code, but it should be noted, the code produced an error which did not plot the associated figure of plume size and Mach numbers. The only value produced was the entrainment ratio of the two flows. The sole presentation of this data is to exhibit how an inviscid simulation should predict the performance and its difference to an experimental flow. The CPSE simulation code is currently being rewritten to account for smaller nozzle geometries and robustness. Figure 59 represents the performance of the CPAAR as a function of pressure ratio and entrainment ratio. 


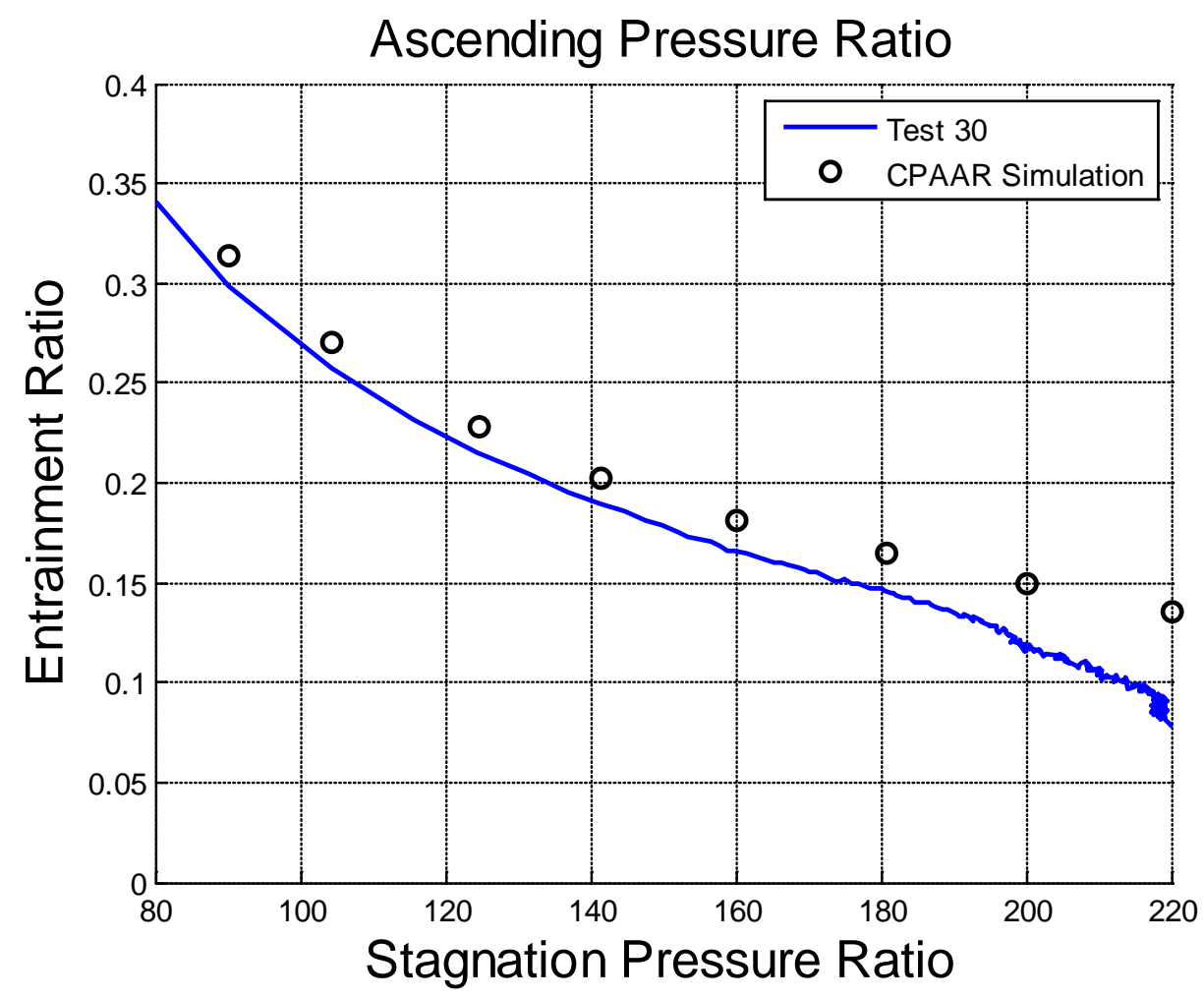

Figure 59: Ejector Performance and CPSE Simulation

The blue line represents the data recorded during the test and the dots are a series of CPSE simulation results. The simulation used the inlet conditions of each pressure ratio point at which it was calculated. As shown, the simulation becomes less accurate as the pressure ratio increases. This is contradictory to earlier simulation samples compared with Gist's data. The primary difference is the much high pressure ratio observed during this test series. The simulation predicts an entrainment ratio about $98 \%$ larger than what as actually observed during the test.

Morham suggests as a result of the small error between his simulations and Gists experimental data, the primary method of momentum transfer is due to pressure gradient effects. The figure above produces data which suggests that as the pressure ratio increases, viscous interactions between the two flows become a much larger driver in 
entrainment ratio reduction which is similar to many studies of ejector performance presented in the past ${ }^{7,8,21}$.

The MATLAB code used for reduction and analysis of the raw data was used to isolate specific instances during each test. The index of pressure ratios in increments of 20, starting at a pressure ratio of 40 , and ending at the maximum pressure ratio was recorded and used to identify the values of the required variables for the simulation input. The input of the simulation were data points recorded during a test, the subsequent plume size and entrainment ratio calculation was then produced and compared to the calculated entrainment ratio from the test data and shown in Table 4.

Table 5: Simulation Error

\begin{tabular}{|c|c|c|c|c|}
\hline & PR (max) & ER & ER (Simulation) & Simulation Error \\
\hline Test 30 & 220.3 & 0.051 & 0.135 & $90.86 \%$ \\
\hline Test 31 & 211.4 & 0.076 & 0.149 & $47.43 \%$ \\
\hline Test 33 & 157.0 & 0.161 & 0.202 & $7.86 \%$ \\
\hline
\end{tabular}

At low Fabri-choke pressure ratios, the simulation has a very small error relative to the experimental data, as initially compared to Gist and verified by this series of testing, with a similar error in the calculation. Although as the pressure ratio is increased, and as the simulation becomes closer to a Fabri-block condition, the error is much higher.

Due to the inviscid assumption of the model, it is unable to calculate the mass transfer between to the two flows along the viscous layer of the plume boundary. 


\section{Conclusions}

The Cal Poly Air-Augmented Rocket test apparatus and cell was rebuilt, and research was restarted. The testing was performed using high pressure gaseous nitrogen. Several modifications were made to the system, including increasing the mixing duct length and addition of a secondary plenum. Several phenomena were observed as a result of the addition of a fixed volume plenum. The experimental results were compared to previous CPAAR results obtained during atmospheric testing along with previous transient analysis of ejector systems. The following conclusions were made based on the experimental results.

1. The addition of a fixed volume plenum produces a transient flow condition in the mixing duct that can be assumed to have a quasi-steady quality once both flows have become established.

2. The smallest startup transient during formal testing was $1.1 \%$ of the total transient flow time. The flow field can be analyzed as steady state under a quasi-steady assumption during the period after the start-up transient region of the secondary flow and is independent of the primary starting transient.

3. The development of the supersonic saturated case in a transient flow regime is dependent on the transient pressurization of the primary flow. If the primary flow is sufficiently quick, the primary flow structure will develop before the secondary flow has become establish, and essentially bypass a saturated condition and begin entrainment in a Fabri-choke mode. 
4. The Fabri-choke case was observed during operation and the location of the Fabri-choking area of the secondary flow was observed in the data. The location of the secondary choke point has a logarithmic relationship with the total pressure ratio. At a pressure ratio of 100, the choke location occurs at approximately 1.5 inches downstream of the nozzle exit. At $P R=220$, the choke location occurs 6 inches downstream from the nozzle exit.

5. The area of the secondary flow choking location was derived mathematically from observed pressure data and apparatus geometry. The location of the Fabri-choke moves downstream in the mixing duct as pressure ratio increases and the secondary flow choking area decreases. The area of the choking location changes from $0.46 \mathrm{in}^{2}$ to $0.27 \mathrm{in}^{2}$ from $\mathrm{PR}=100$ to $\mathrm{PR}=220$.

6. The rate of change of the Fabri-choke location downstream is linearly related to the reduction in area of the Fabri-choke location during transient operation.

7. The low stagnation pressure and high speed of the secondary flow aids the collapse of the quasi-steady transient primary flows shock structure as primary flow momentum decreases.

8. The plenum produces consistent results and increases the rate of pressure ratio growth compared to atmospheric tests and produces results capable of being analyzed as quasi-steady with steady state assumptions. The plenum is capable of achieving pressures below 5 psia, and achieved a minimum pressure of 3.3 psia during operation. To date, the plenum has recorded over 70 evacuation and pressurization cycles. 
9. Asymmetry of the flow becomes more pronounced at higher pressure ratios and creates a bias towards the collapse of the primary plume shock structure.

10. Blocking of the right secondary duct occurs due to an asymmetry of the flow. As the right duct is blocked, the left duct becomes a region of instability.

11. At high pressure ratios, the asymmetry causes the un-choke process of the secondary flow to occur at a much slower characteristic rate.

12. As pressure ratio increases, the viscous interactions of the two flows become a larger driver in entrainment. At low secondary flow rates, mass transfer occurring between to the two flows becomes more dependent on the viscous layer of the plume boundary.

13. The CPSE simulation is unable to accurately predict AAR performance when its inputs are changed from the original Cal Poly AAR configuration. At high pressure ratios $(\mathrm{PR}=220)$, the error in the prediction is $90 \%$. 


\section{Future Work}

The research done in this series of testing has produced valuable data for the CPAAR project in understanding the performance of supersonic ejectors at high pressure ratios. The modifications made to the test apparatus was done based on suggestions as a result of previous research on the facility. To further study the flow-field in a supersonic mixerejector, further modifications and research can be done to supplement the study of the CPAAR to validate research for air-augmented rocket systems.

As suggested in previous research ${ }^{11}$, viscous interactions should be integrated into the simulation capabilities. The present research has provided data to use as verification of any viscous interactions additions to the CPSE simulation. A viscous model would be able to produce predictions of entrainment and secondary flow data with greater accuracy.

The largest issue which needs to be addressed with relation to the testing of the apparatus is the transient flow of the primary and secondary flows. The transient flow of the primary reduces the total time supersonic flow can be produced and the maximum pressure ratio attainable, the addition of a high pressure, high mass flow regulator $(>2.5$ $\mathrm{lb} / \mathrm{sec}$ ) for the primary flow would allow the rocket to be operated at various flow-field conditions. A flow regulation system for the secondary flow would also allow for further manipulation of the flow field at the discretion of the research. By producing results which are steady-state, the data produced would be of greater fidelity in comparison to a simulation code and produce results closer to actual AAR performance and flight conditions. 
Along with methods of flow regulation, increasing the volume of the plenum and an ability to reduce the pressure in the secondary flow before the test begins would reduce the startup transient behavior of the current tests. The best method of this is completely encasing the apparatus in a large volume that can be reduced to a certain vacuum level. Also, a study of the asymmetry in the flow should be done to determine what the exact contributing factors are, and the degree in which they affect the asymmetry observed in this and previous research.

Lastly, CPAAR needs to be fired under hot fire conditions. The addition of heat transfer effects to the simulation and hot fire tests would produce data which is much more applicable to actual AAR operation. The data obtained from hot-fire tests and simulations could be correlated to previous cold flow tests and present the relationship of cold flow research to hot fire research and actual AAR performance. 


\section{References}

1. Lehman, Milton, This High Man: The Life of Robert H. Goddard, New York, NY: Farrar, Strauss, and Co., 1963

2. Miller, Jay, The $X$-Planes: $X-1$ to $X$-45. Hinckley, England: Midland Publishing, 2001

3. Merlin, Peter. Mach 3+: NASA USAF YF-12 Flight Research 19691979. Washington, D.C.: Diane Publishing Co., NASA History Division Office, 2002

4. Gist, D.R., "Examination of Flow Field Characteristics and Fabri-Choking of a 2-D Augmented Rocket”, Master's Thesis, Department of Aerospace Engineering, Cal Poly, San Luis Obispo, CA, 2007.

5. Fabri, J., \& Paulon, J., “Theory and Experiments on Supersonic Air-to-Air Ejectors”, NACA TM 1410, Sept 1956

6. Fabri, J., and Siestrunk, R., "Supersonic Air Ejectors", Advances in Applied Mechanics, Vol. 5, Von Karman and Dryden, editors, Academic Press, 1958, pp. 135.

7. Addy, L.A., "On the Steady State and Transient Operating Characteristics of Long Cylindrical Shroud Supersonic Ejectors”, University of Illinois, Ph. D. dissertation. University Microfilms, Inc. Ann Arbor, MI., 1963.

8. Lijo, V., Kim, H. D., Rajesh, G., and Setoguchi, T., "Numerical simulation of transient flows in a vacuum ejector-diffuser system”, ISSN 0954-4100, Proceedings of the Institution of Mechanical Engineers. Part G, Journal of Aerospace Engineering, 2010.

9. Foster, T.J., “Rectangular Ducted Methane/GOX Thruster”, Master's Thesis, Department of Aerospace Engineering, Cal Poly, San Luis Obispo, CA, 2008.

10. D. Gist, T. Foster and D. DeTurris, "Examination of Fabri-Choking in a Simulated Air Augmented Rocket," AIAA 2007-5392, 43rd AIAA/ASME/SAE/ASEE Joint Propulsion Conference, July 2007.

11. Morham, B.G., "Numerical Examination of Flow Field Characteristics and Fabri Choking of 2-D Supersonic Ejectors”, Master's Thesis, Department of Aerospace Engineering, Cal Poly, San Luis Obispo, CA, 2010.

12. Anderson, J.D., Modern Compressible Flow, McGraw-Hill Book Company, United States of America, 1982.

13. Brown, C., St.Clair, R., \& Bulman, M. "Kplume Modeling for Ejector Plume Modeling”, written for ISTAR at NASA Marshall Space Flight Center, November 2003.

14. Selvy, B. "Development of an Air Augmented Rocket Plume Test Apparatus” Senior Project, Cal Poly Aerospace Engineering Dept., San Luis Obispo, CA., 2003.

15. Chattopadhyay, C., Pressure Vessels: Design and Practice, CRC Press, 2004.

16. Bednar, H.H., Pressure Vessel Design Handbook, Van Nostrand Reinhold, 1986.

17. Omega Engineering, $P X-302$ Pressure Transducer Specifications Sheet. Website: http://www.omega.com/Pressure/pdf/PX302.pdf, 2006.

18. Omega Engineering, Revised Thermocouple Reference Tables: Type K. Website: http://www.omega.com/temperature/Z/pdf/z218-220.pdf, 2006. 
19. Sutton, George P. \& Biblarz, Oscar, "Rocket Propulsion Elements", John Wiley \& Sons, Inc., New York, 2001.

20. Zucrow, M.J. \& Hoffman, J.D., Gas Dynamics, Wiley Publishing, New York, 1976.

21. Montre, T., "Experimental Investigation of a 2-D Air augmented Rocket: Effects of Nozzle Lip Thickness on Rocket Mixing and Entrainment”, Master's Thesis, Department of Aerospace Engineering, Cal Poly, San Luis Obispo, CA, 2011. 


\section{Appendix}

\section{MATLAB Raw Test Data Manipulation Code}

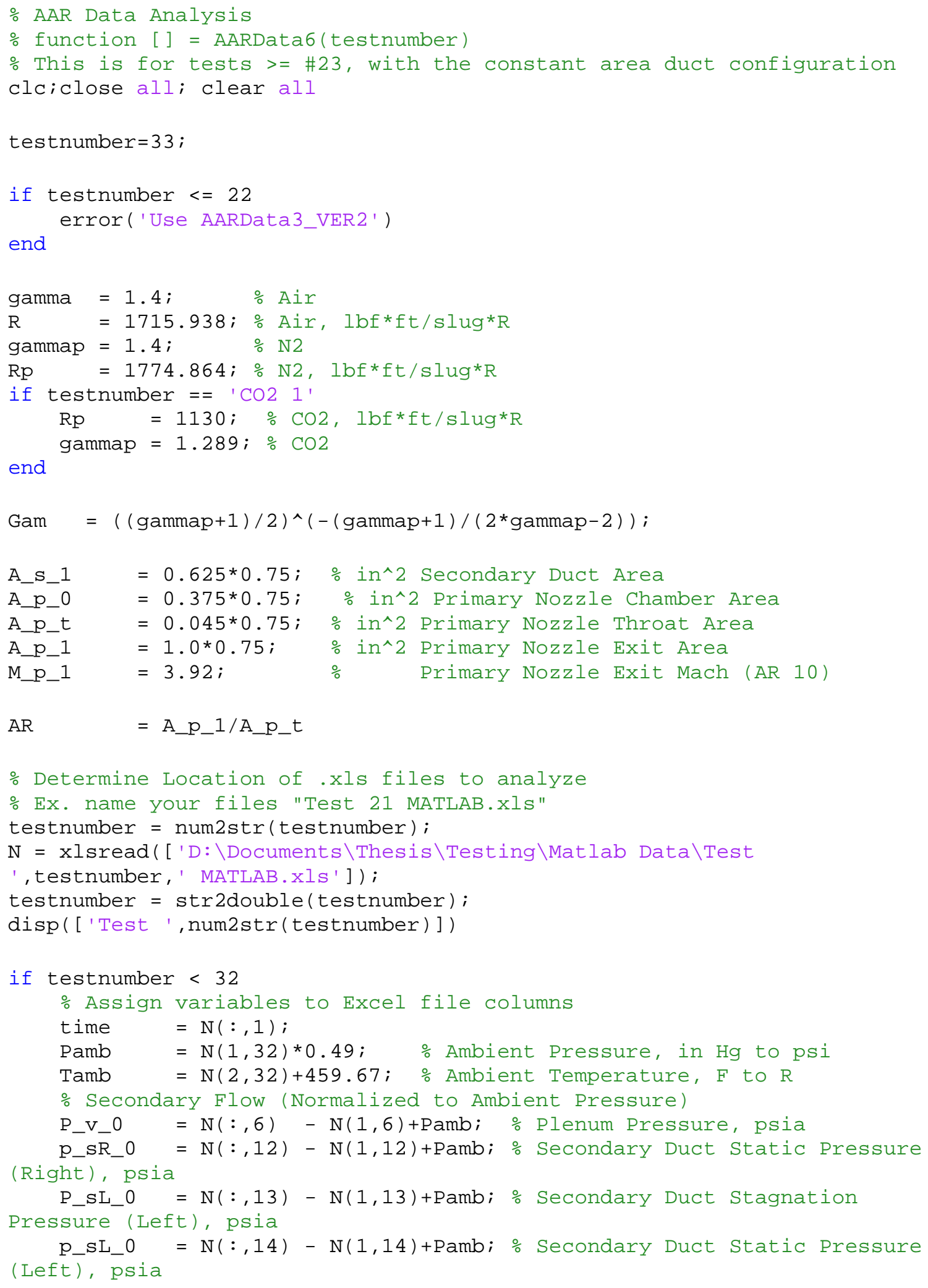




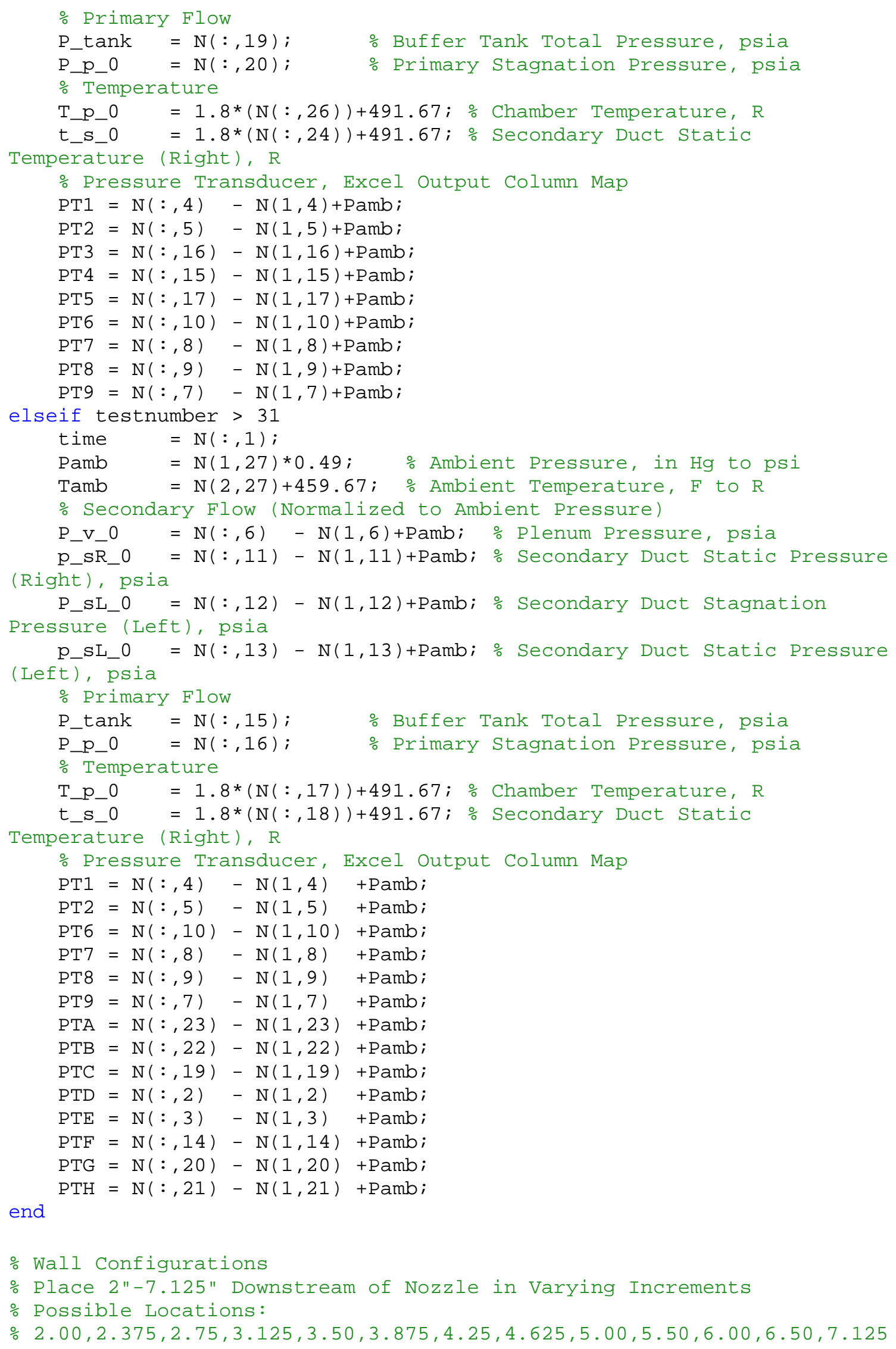




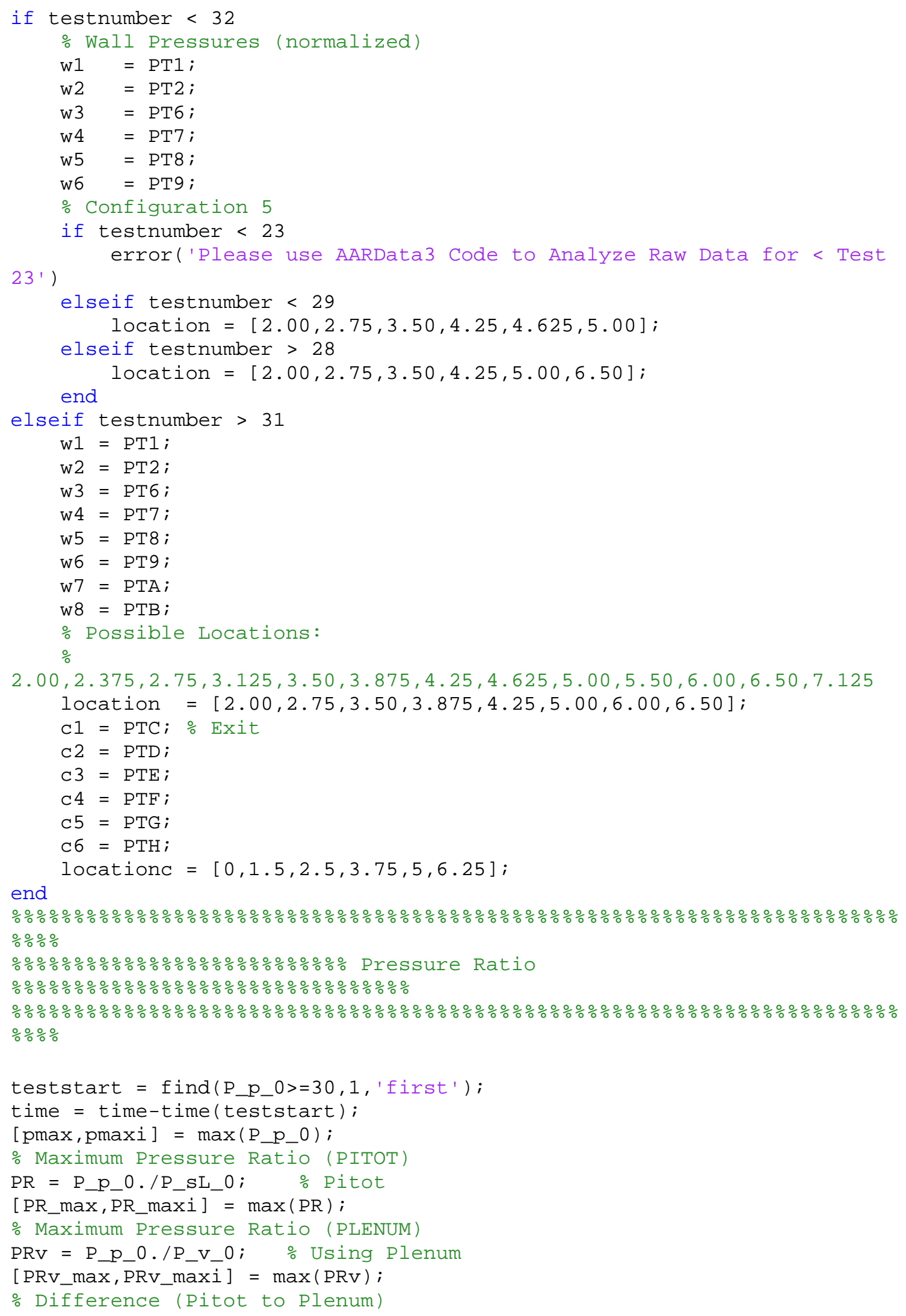




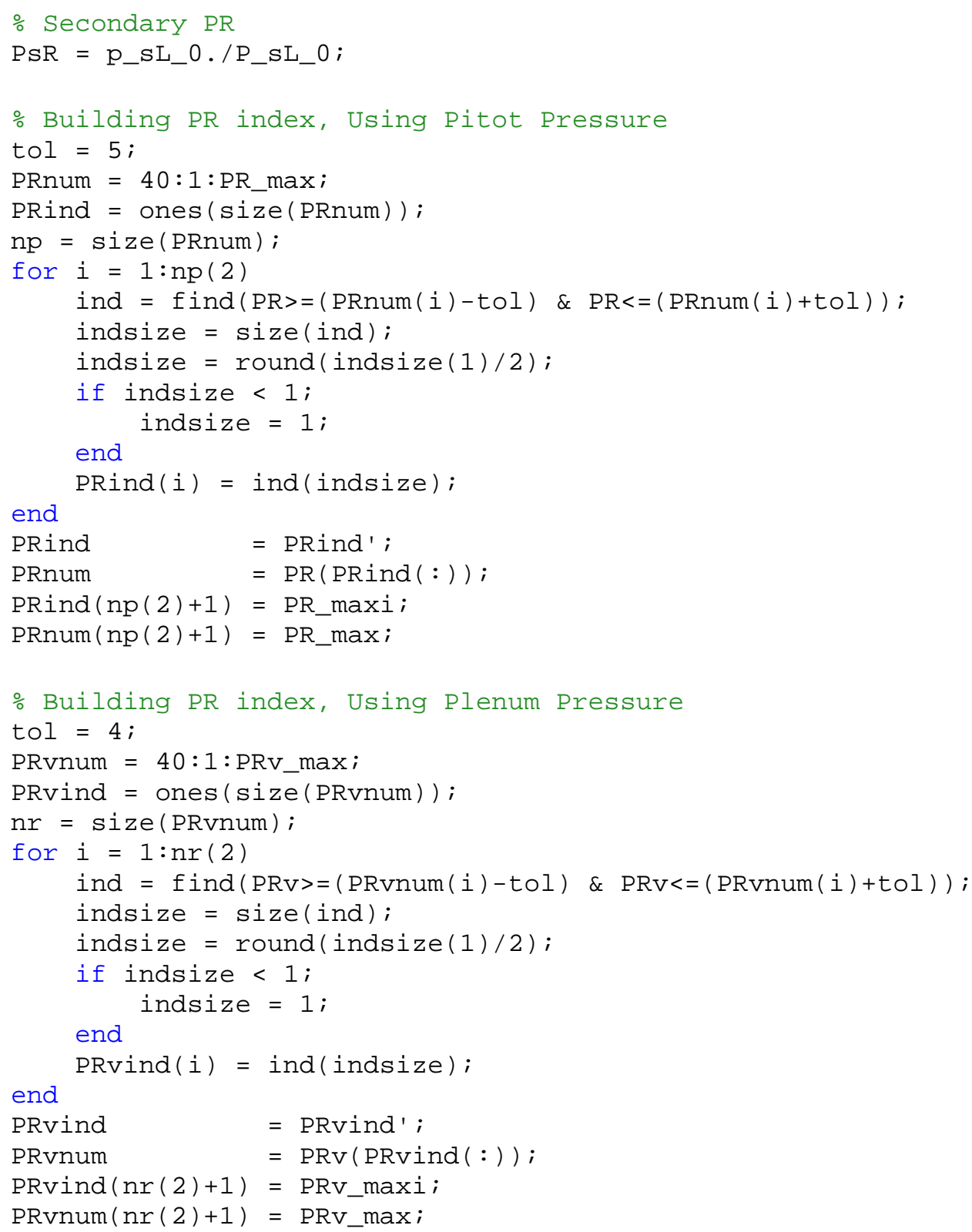

\%\%\%\%\%\%\%\%\%\%\%\%\%\%\%\%\%\%\%\%\%\%\%\%\%\%\%\%\%\%\%\%\%\%\%\%\%\%\%\%\%\%\%\%\%\%\%\%\%\%\%\%\%\%\%\%\%\%\%\%\%\%\% $\% \% \%$ 


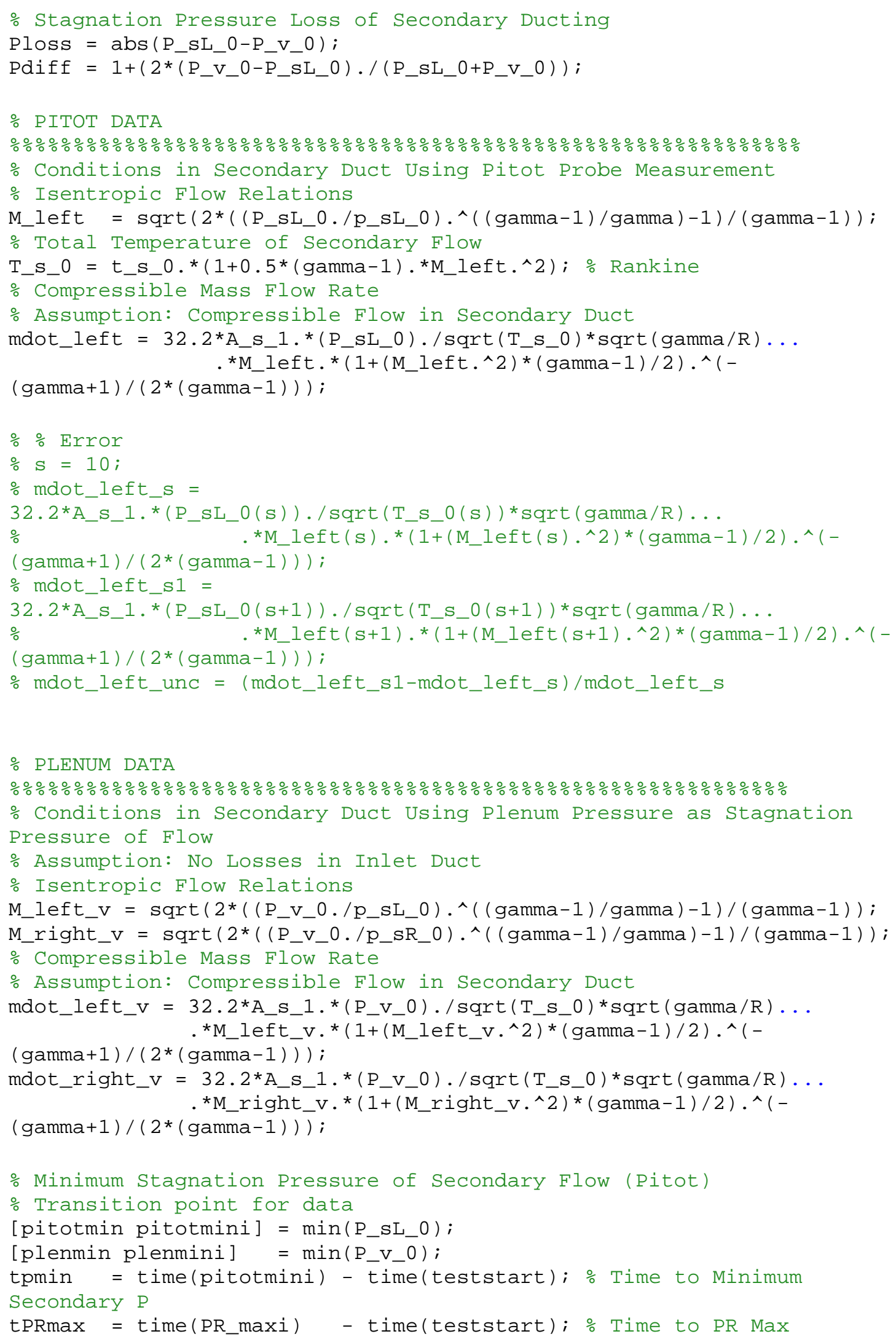


\% Total Transient Flow Time

\% From teststart to P_p_omaxi is Starting Transient Region

\% From P_p_omaxi to Pitotmini is Quasi-Steady Transient Region

tran_t $=$ time $($ pitotmini)-time $($ preteststart $) ; \quad \%$ Total Transient ( seconds)

tran_s = time $\left(P_{-}\right.$_p_omaxi)-time $($preteststart $) ; \%$ Starting Transient ( seconds)

tran_q = time $($ pitotmini $)-\operatorname{time}\left(P \_p \_\right.$omaxi $) ; \quad \%$ Quasi-Steady Region ( seconds)

$\operatorname{disp}([$ 'Total Time $=$ ', num2str(tran_t)] )

disp(['Start-up Transient (\%) = ', num2str(100*tran_s/tran_t), '\%'])

$\operatorname{disp}([' T e s t$ Transient $(\%)=$ ', num2str(100*tran_q/tran_t), '\%'])

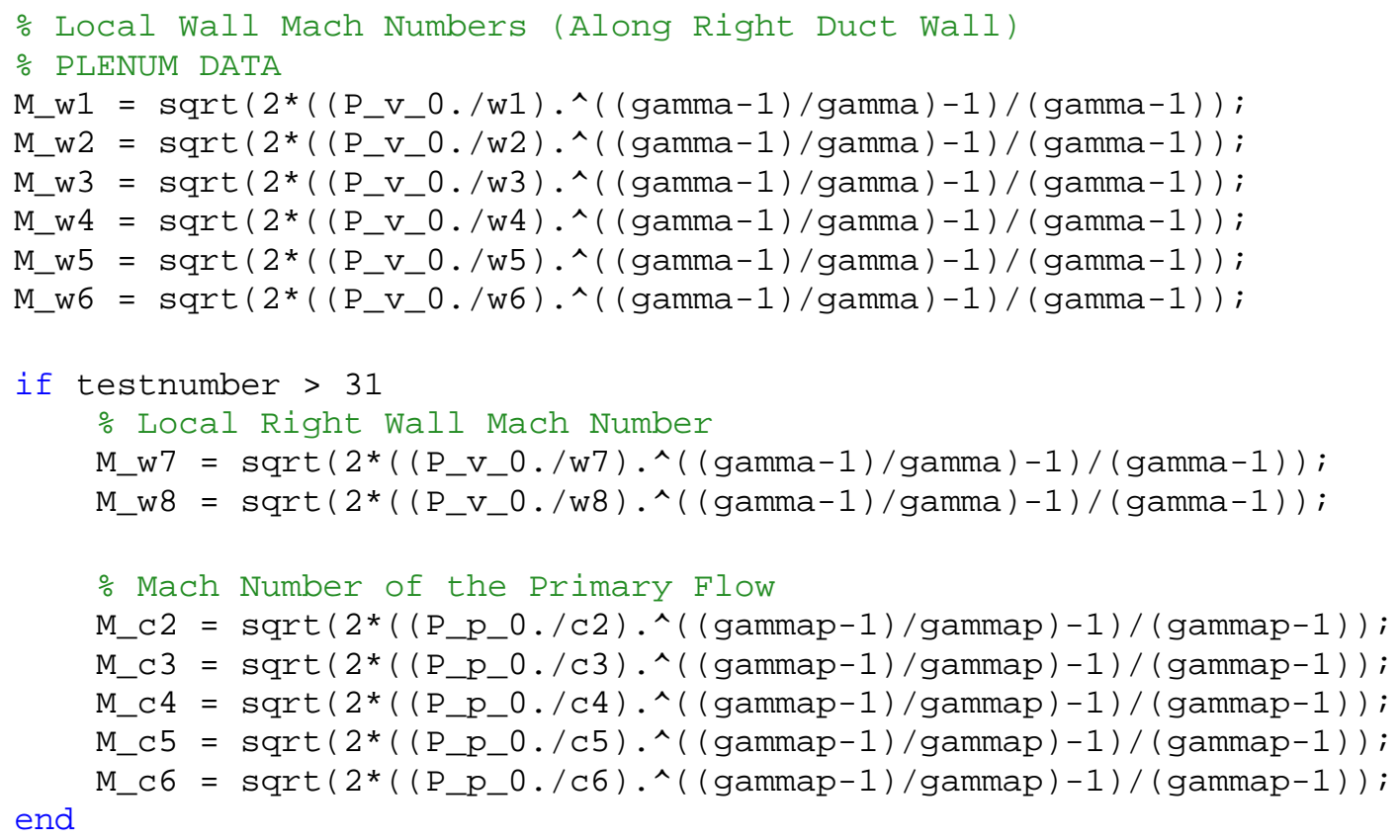




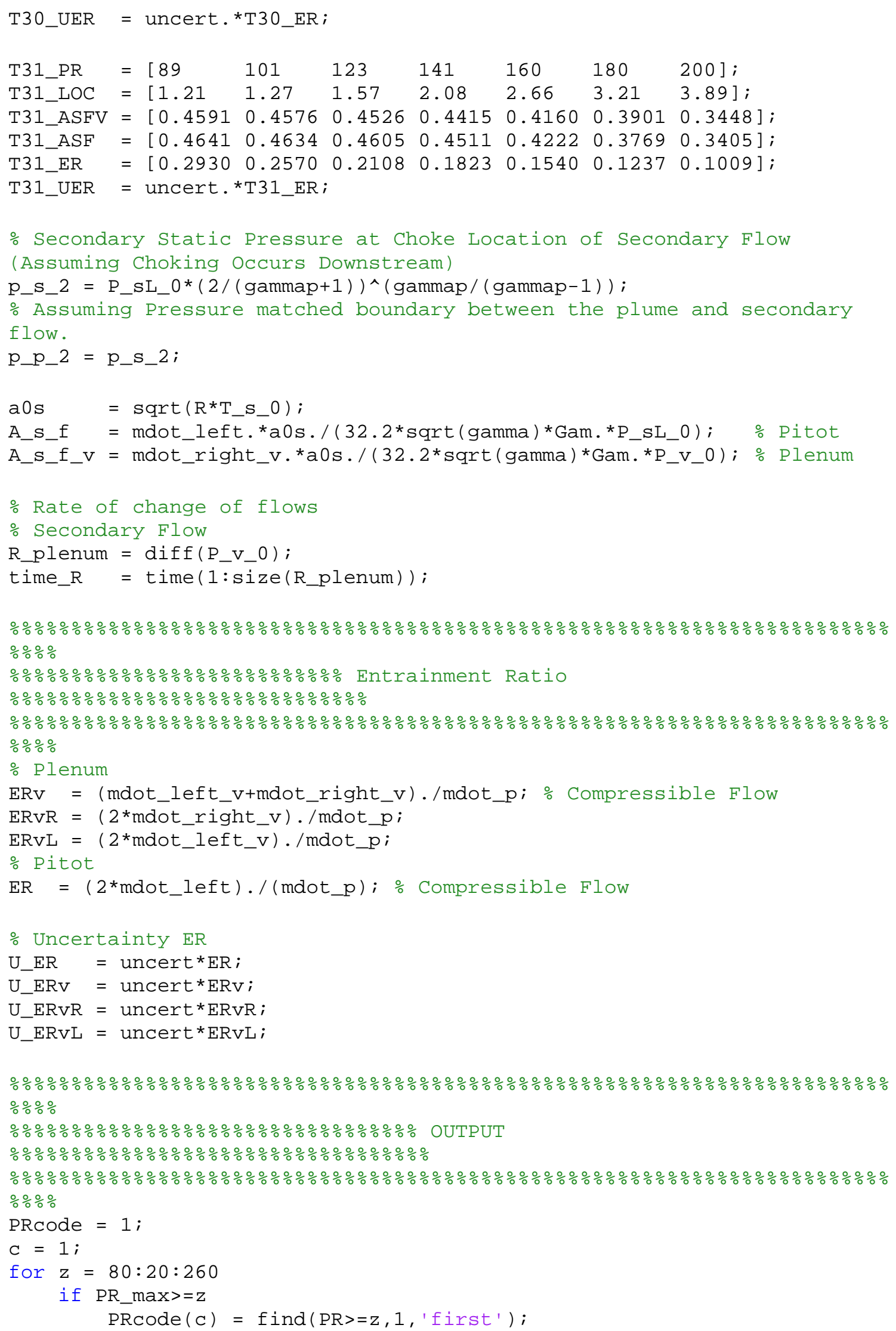




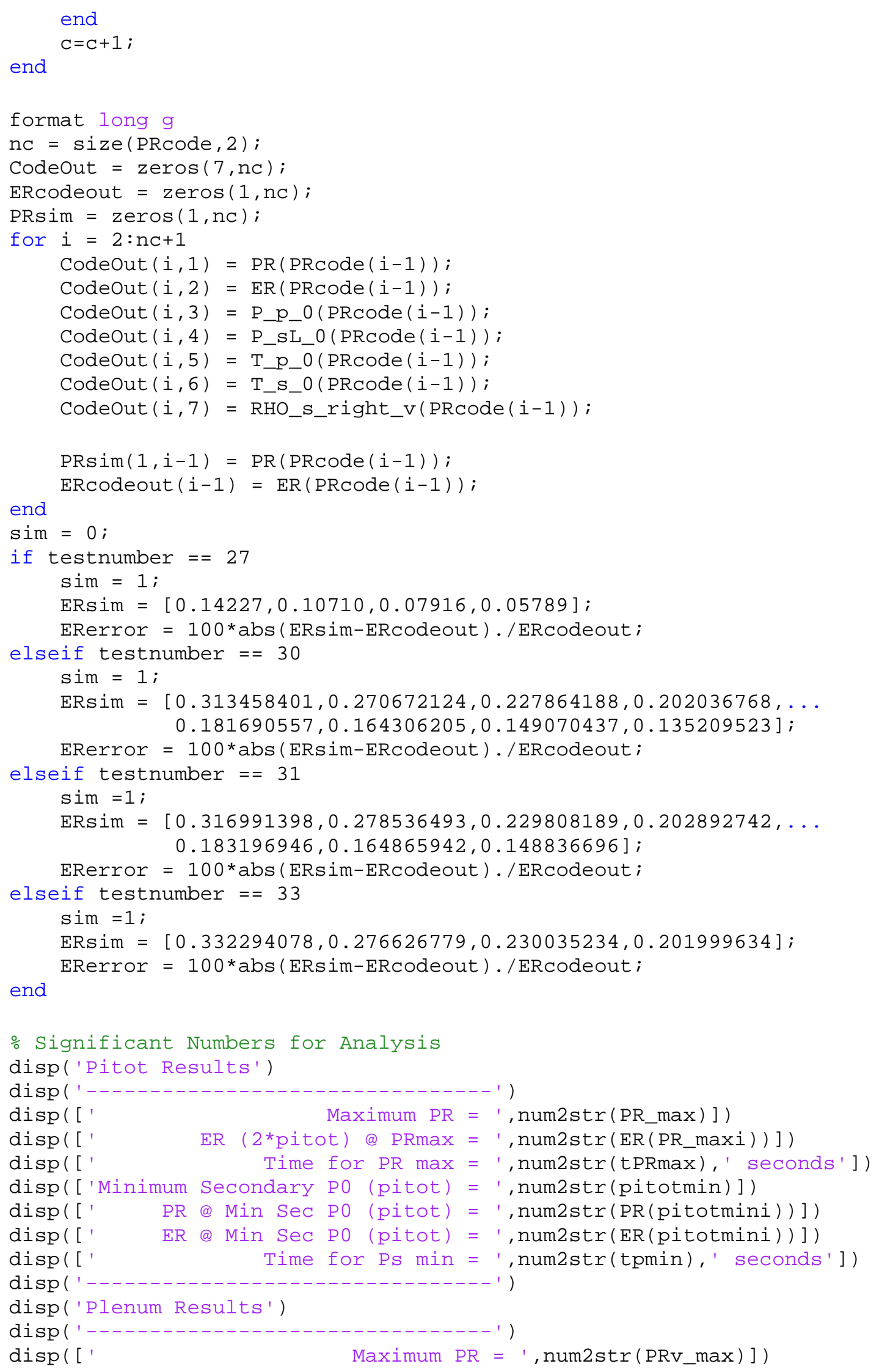




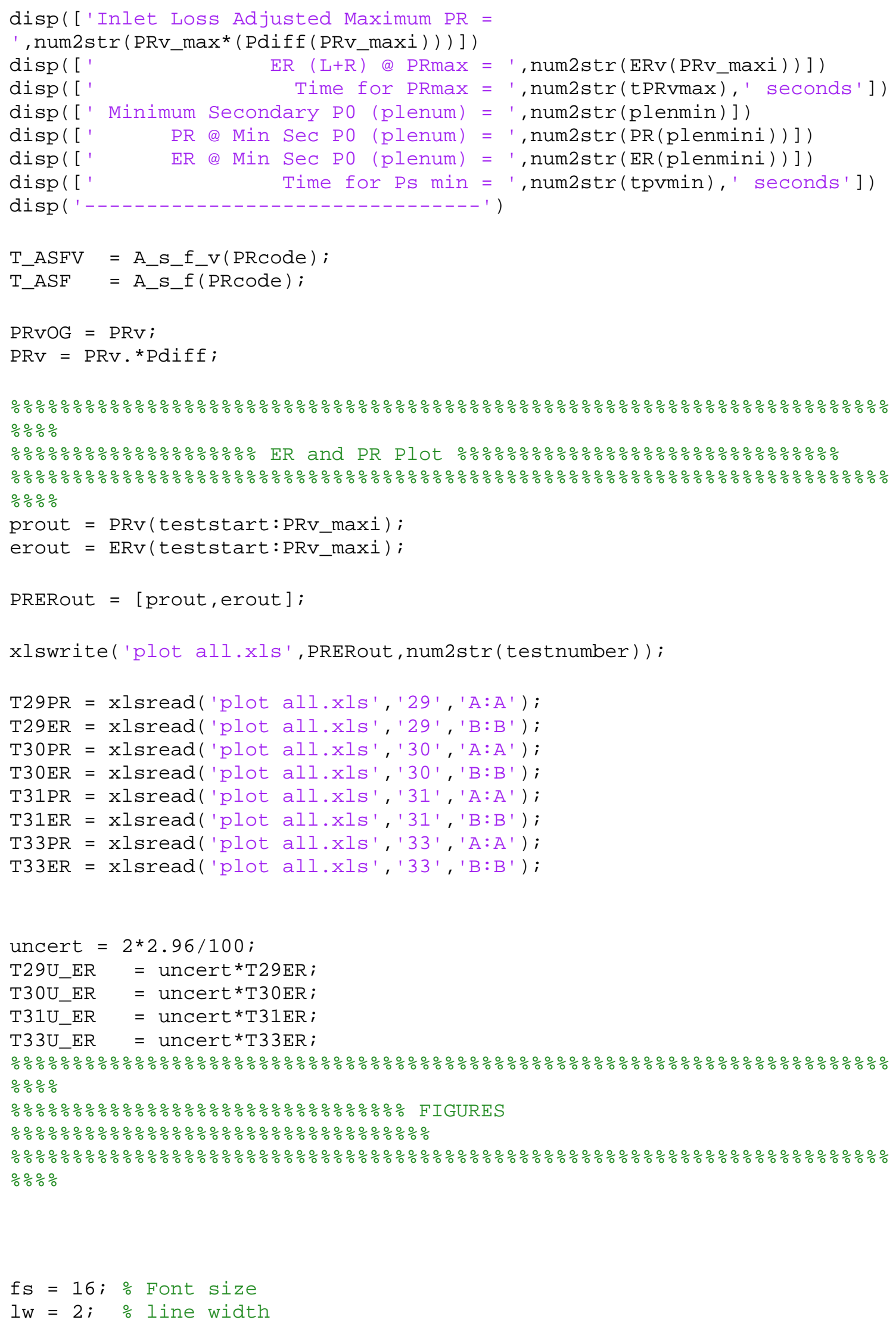




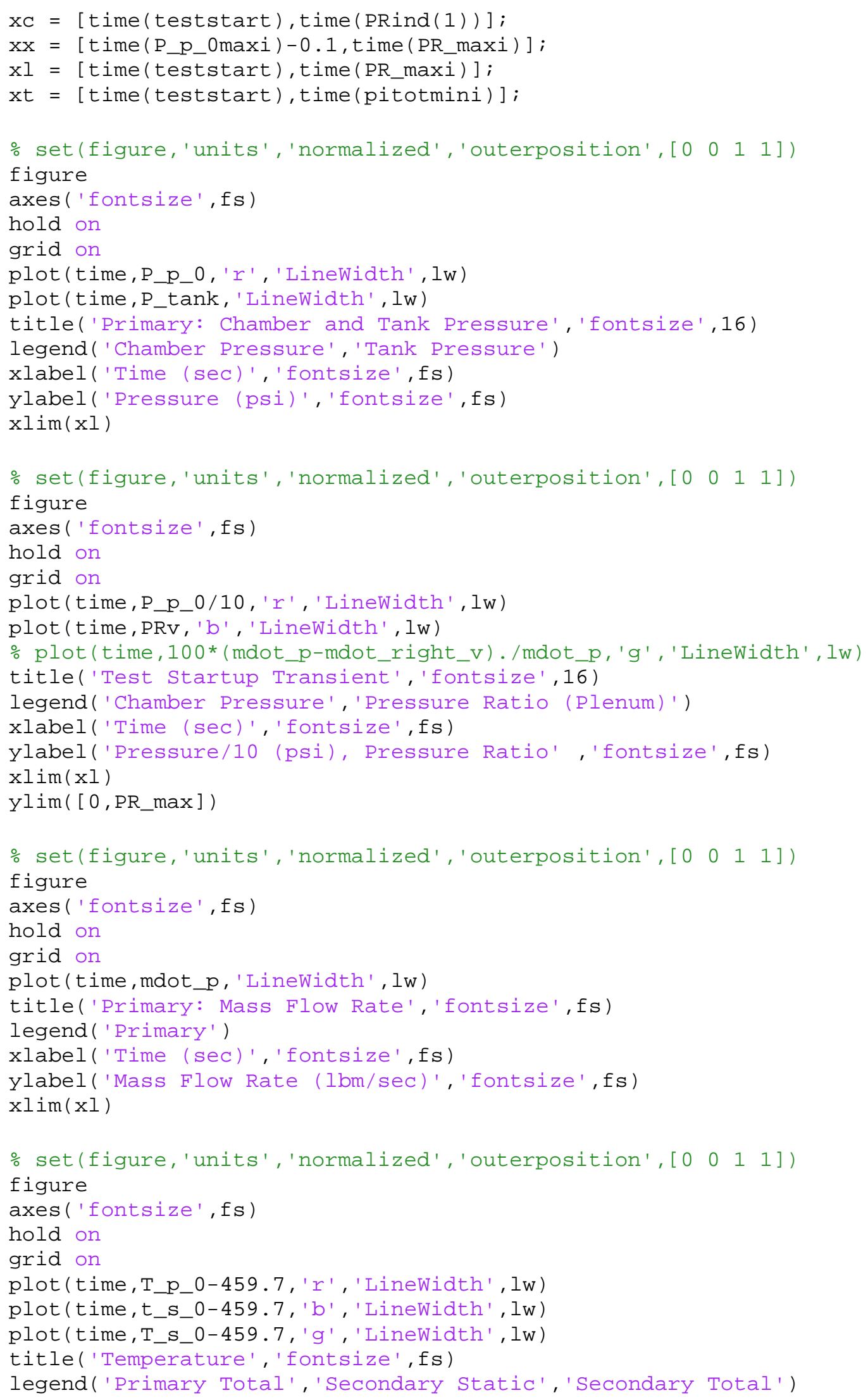




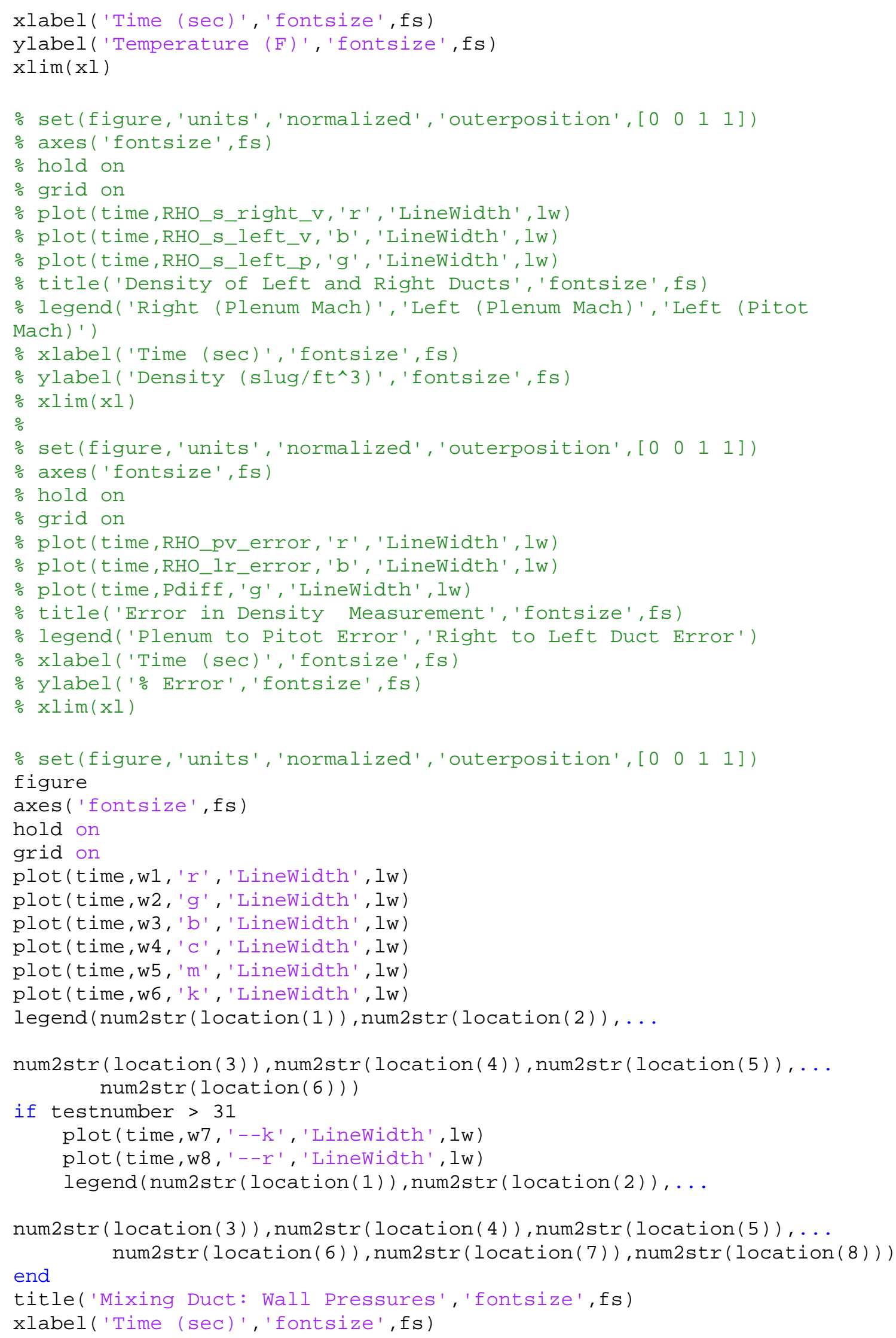




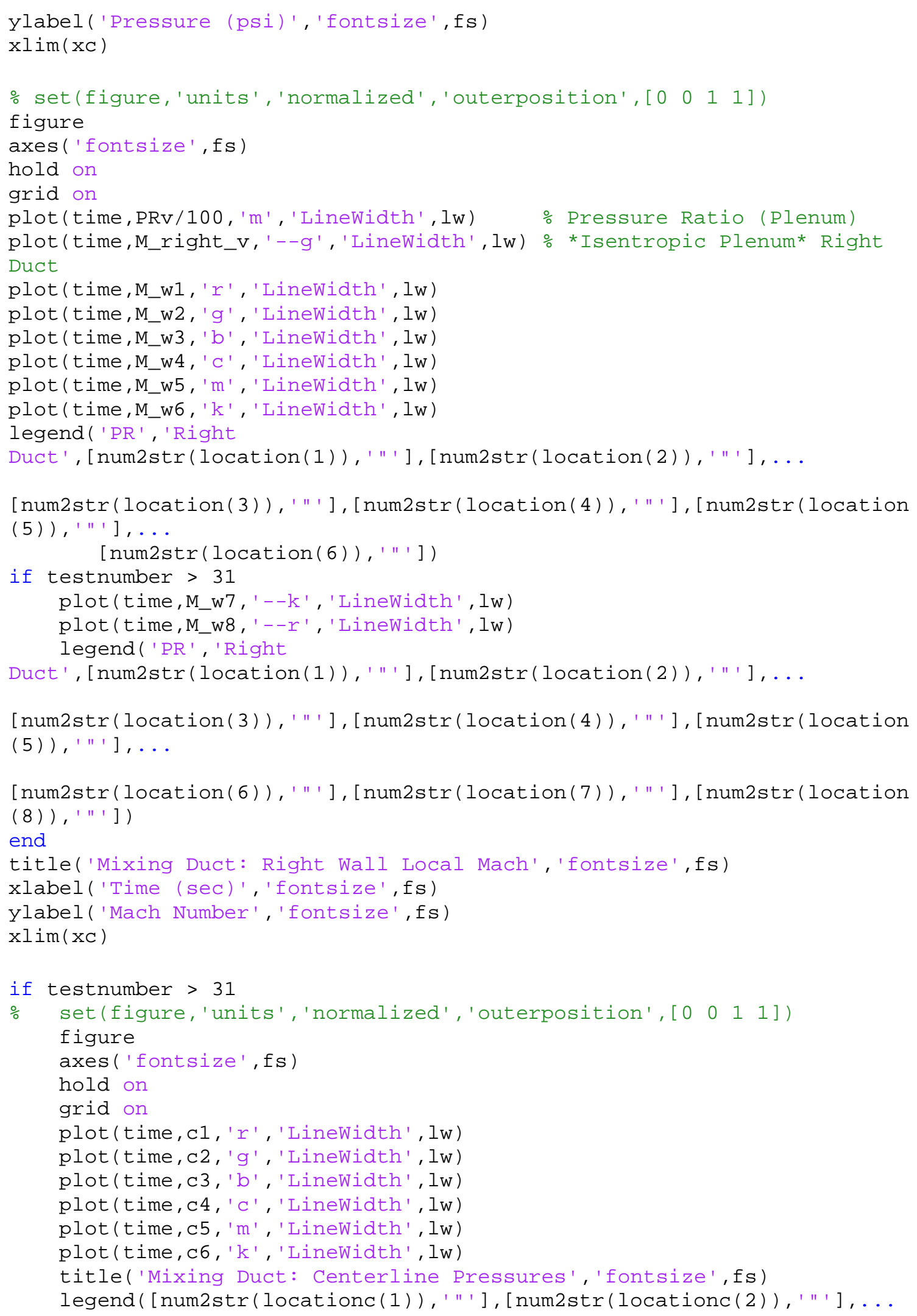




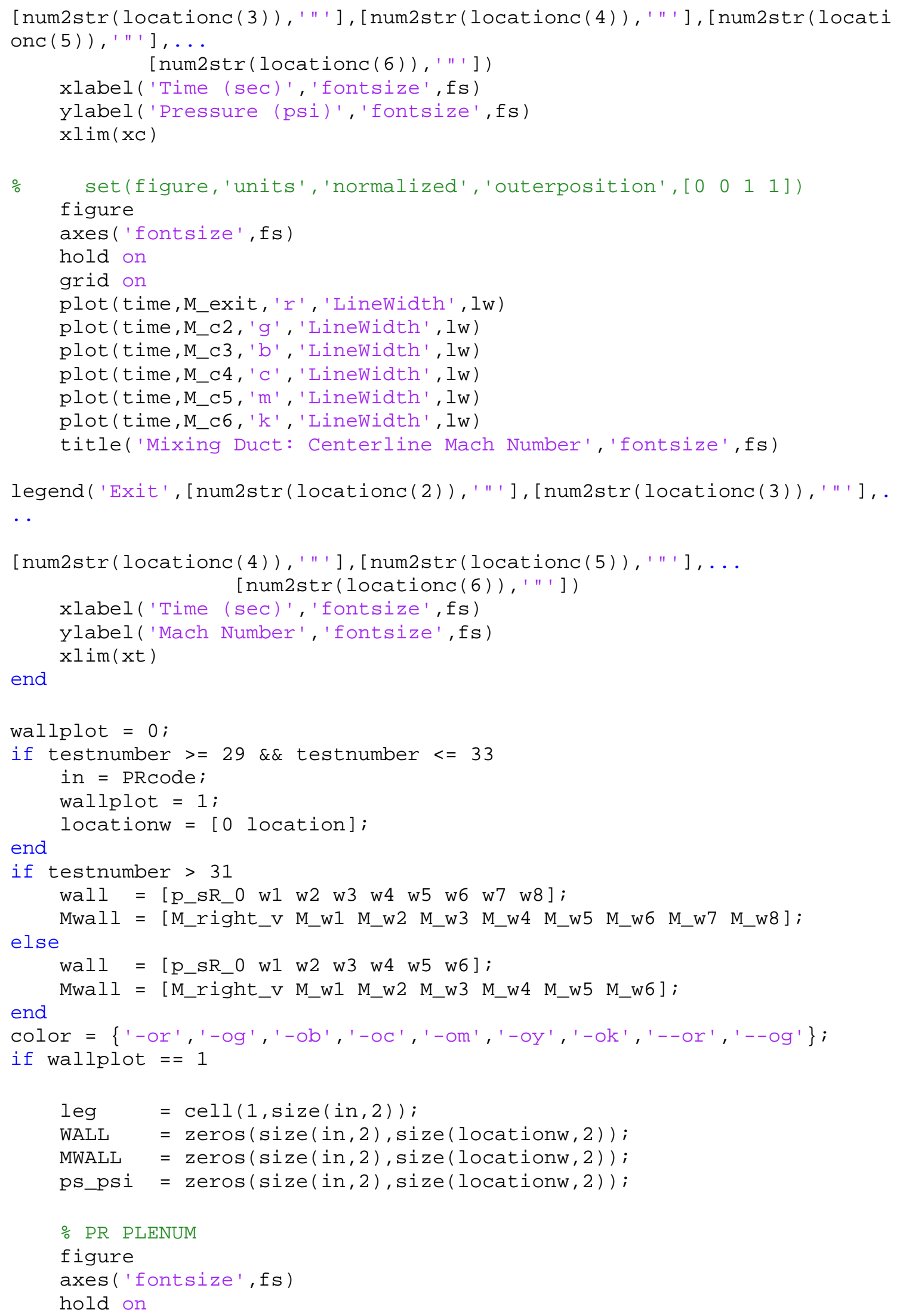




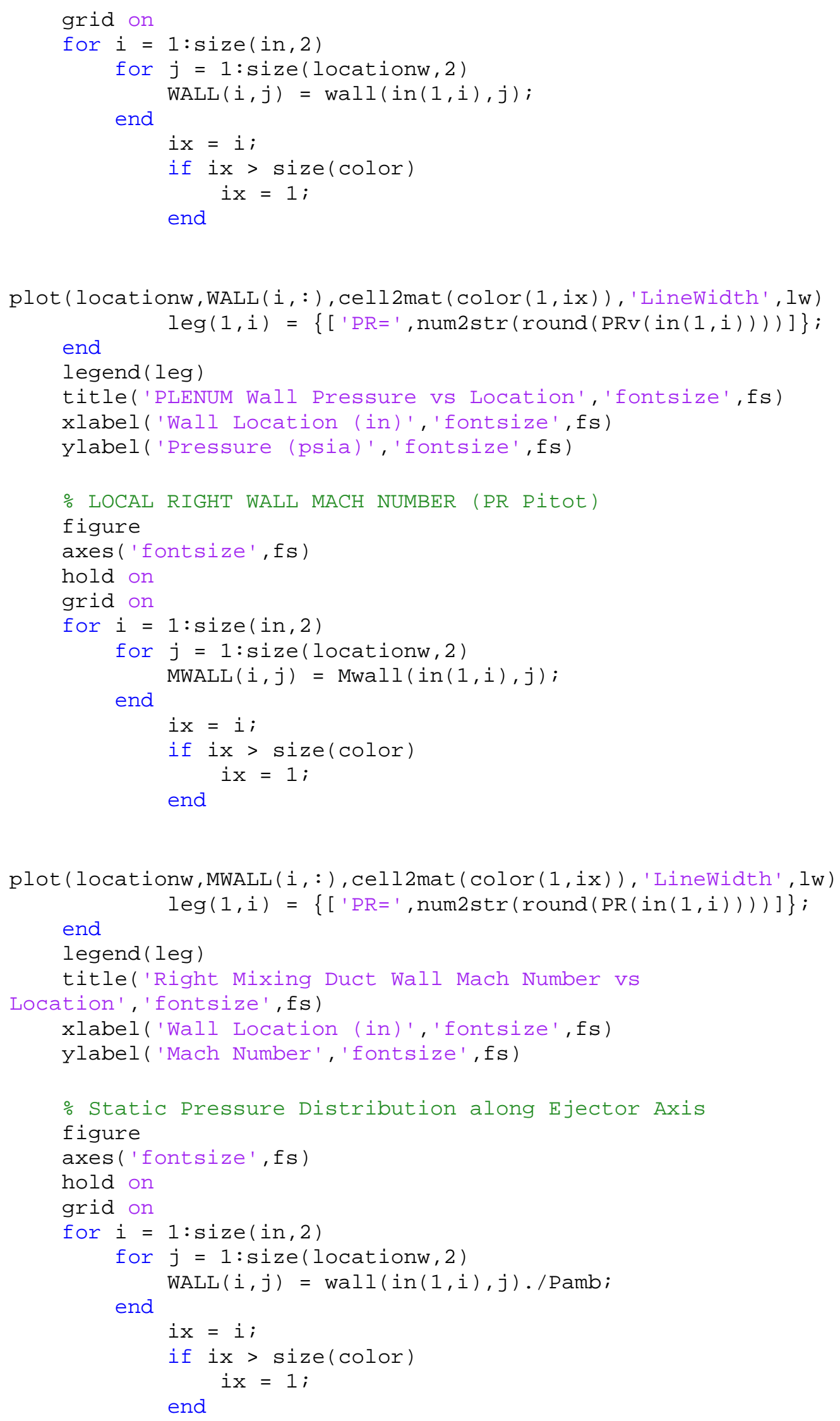




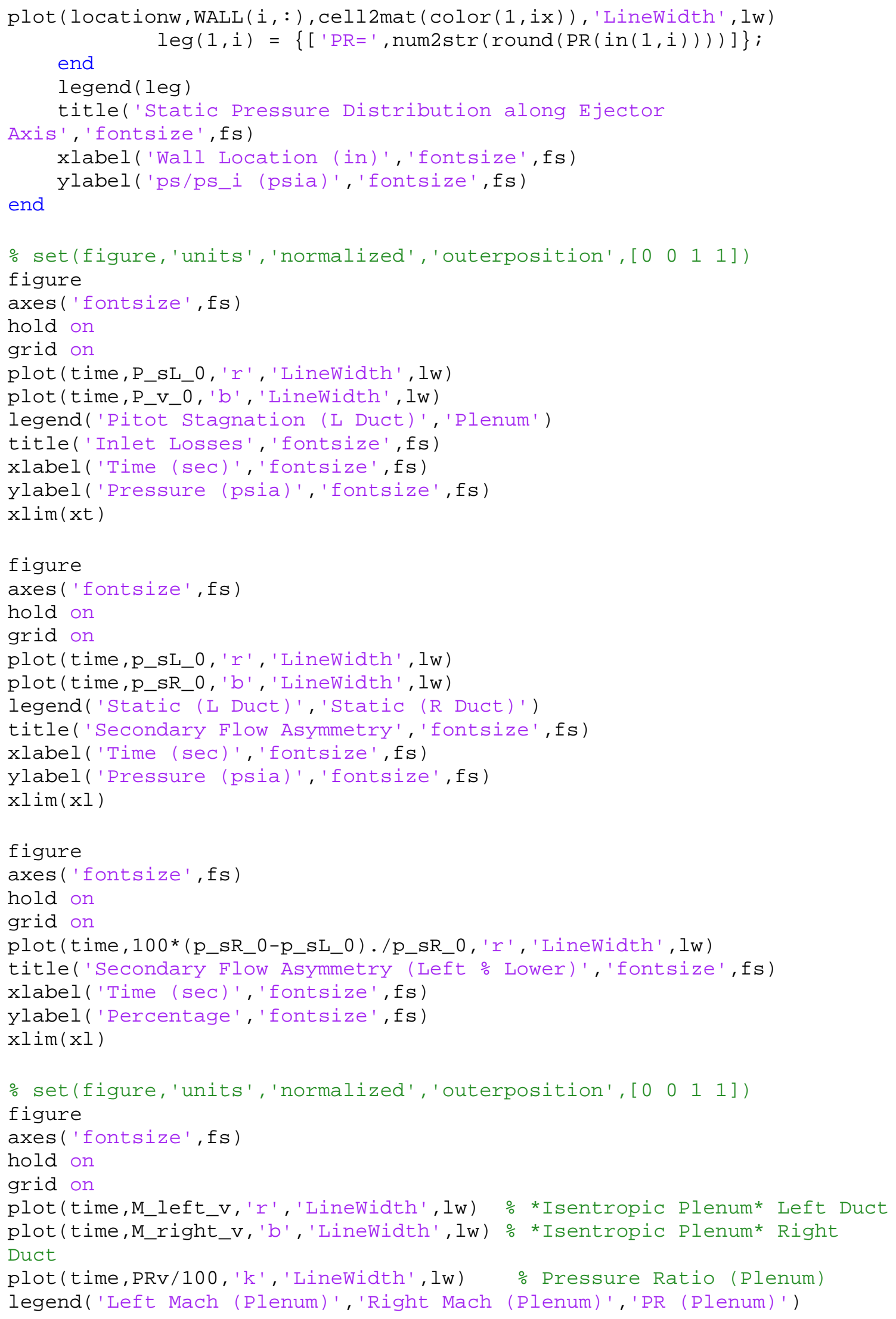


title('Secondary Mach Number and Total Pressure Ratio','fontsize',fs)

xlabel('Time (sec)','fontsize',fs)

ylabel('Mach Number or Total Pressure Ratio/100', 'fontsize',fs)

$x \lim (x l)$

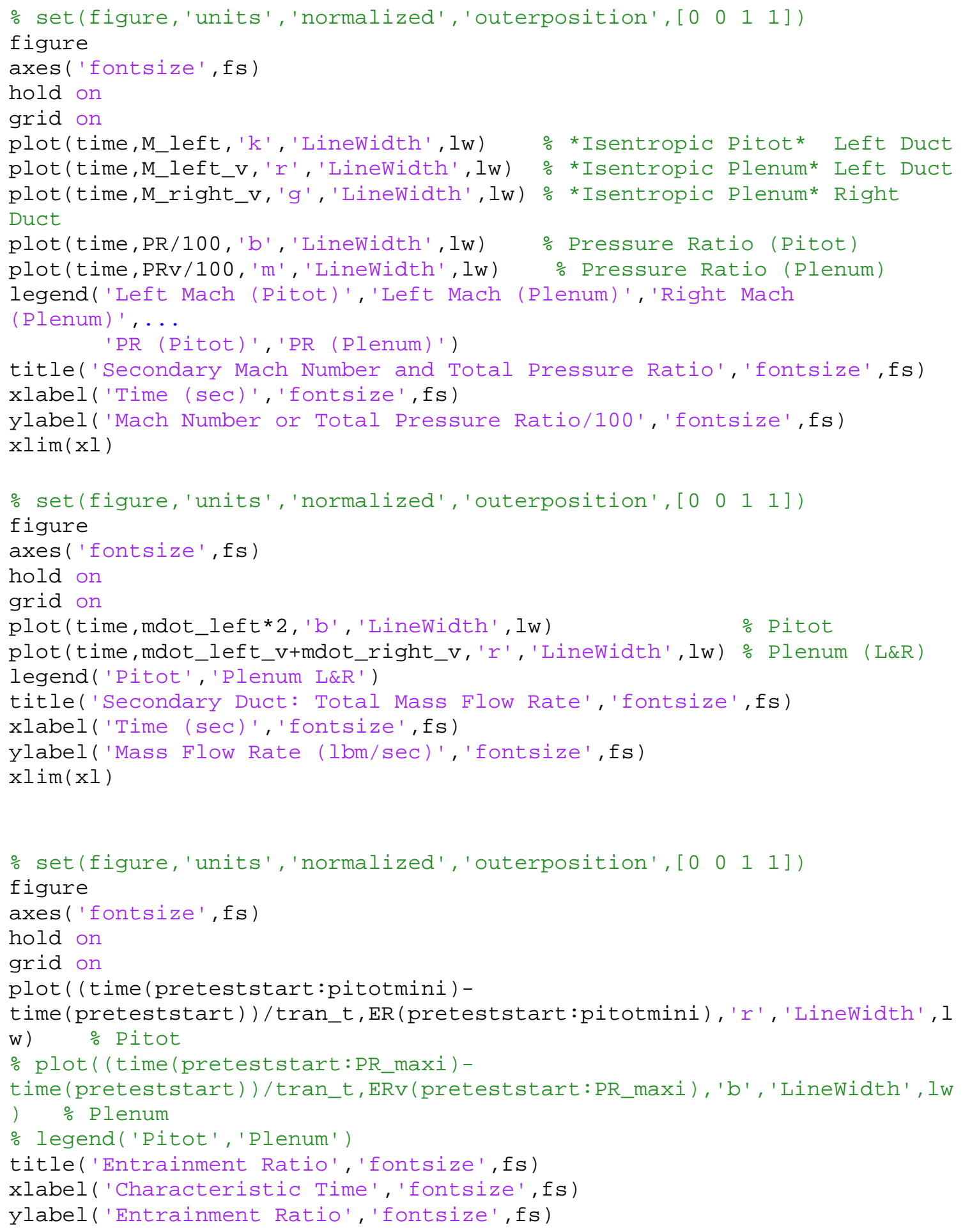




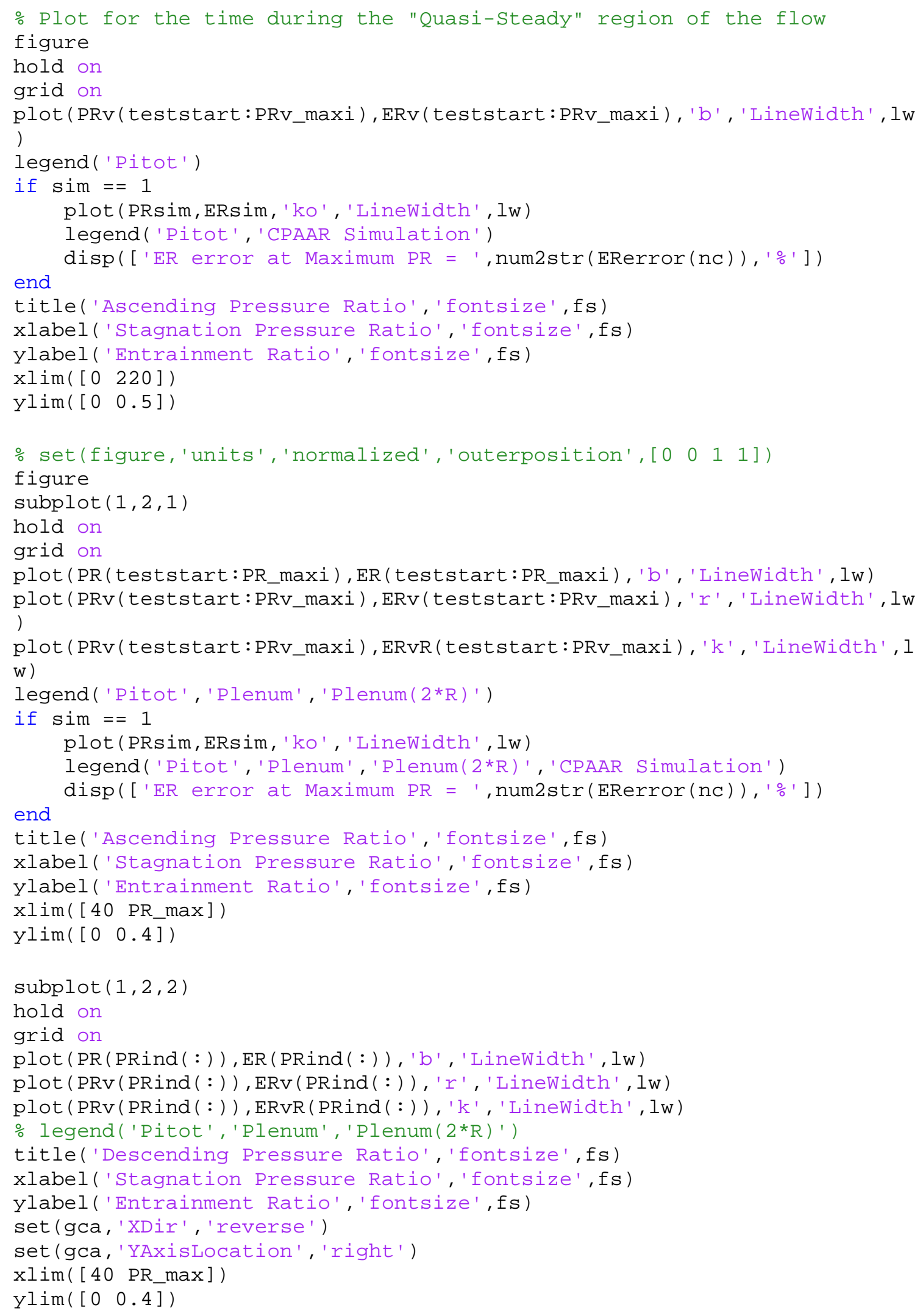




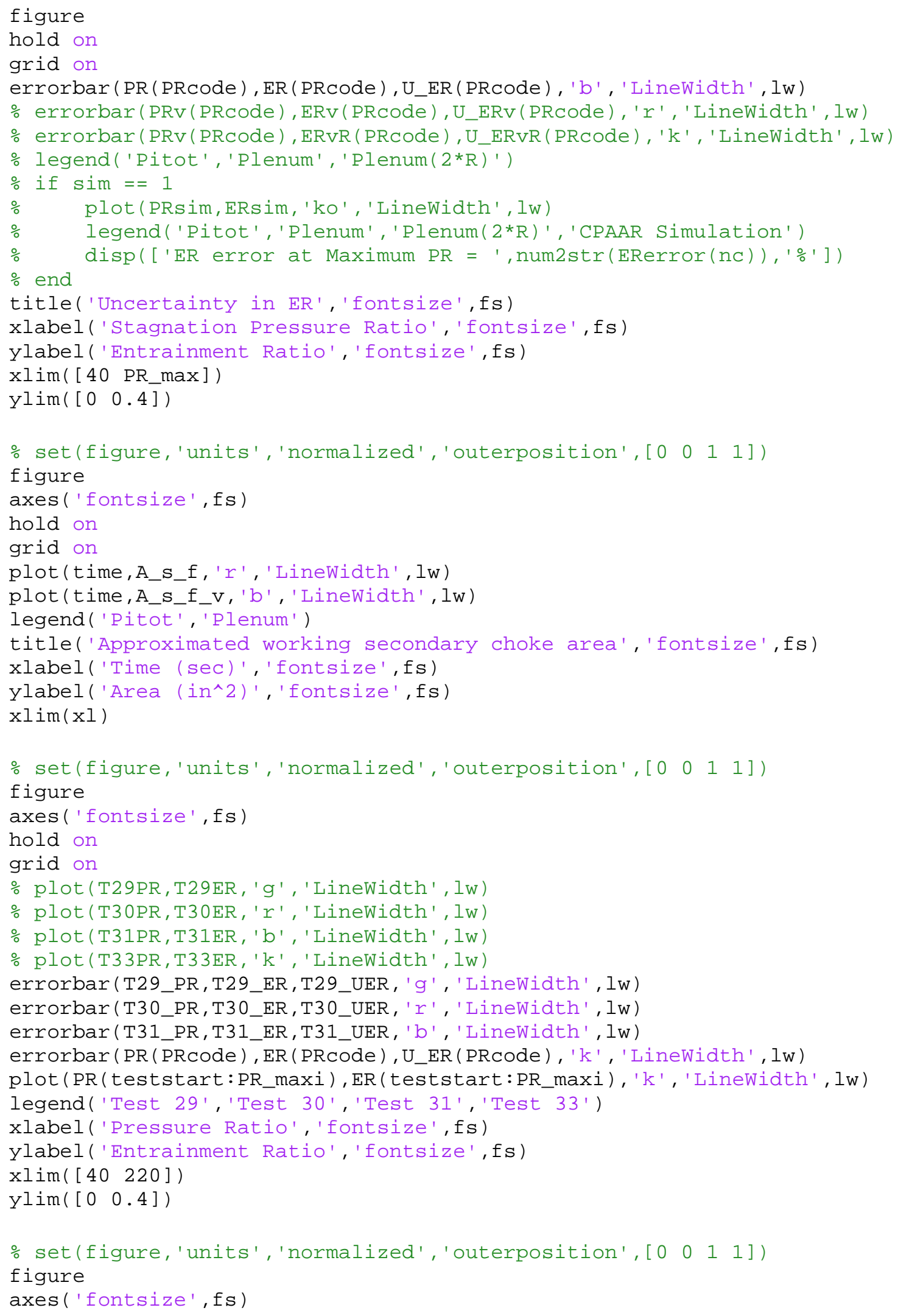




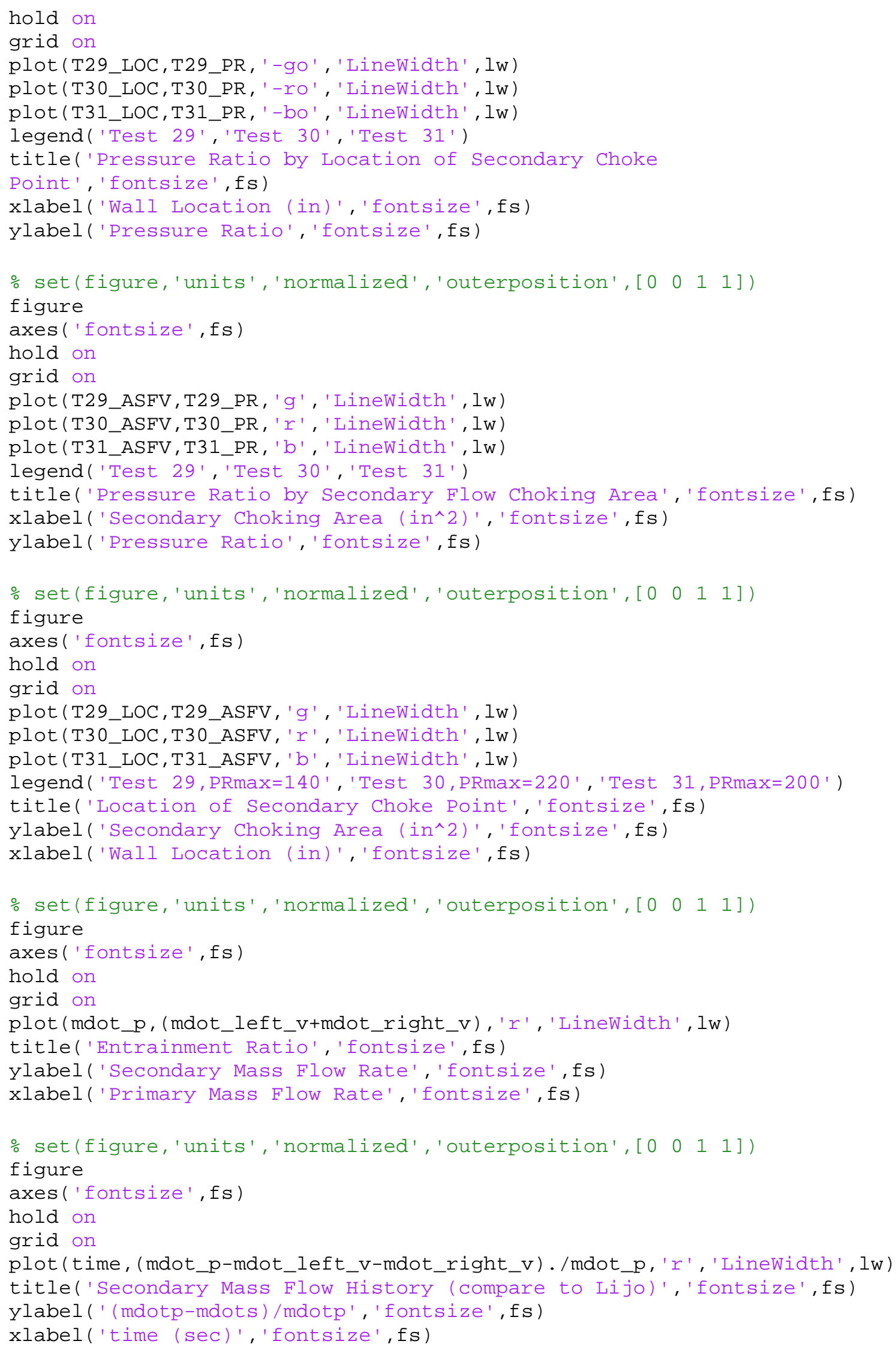




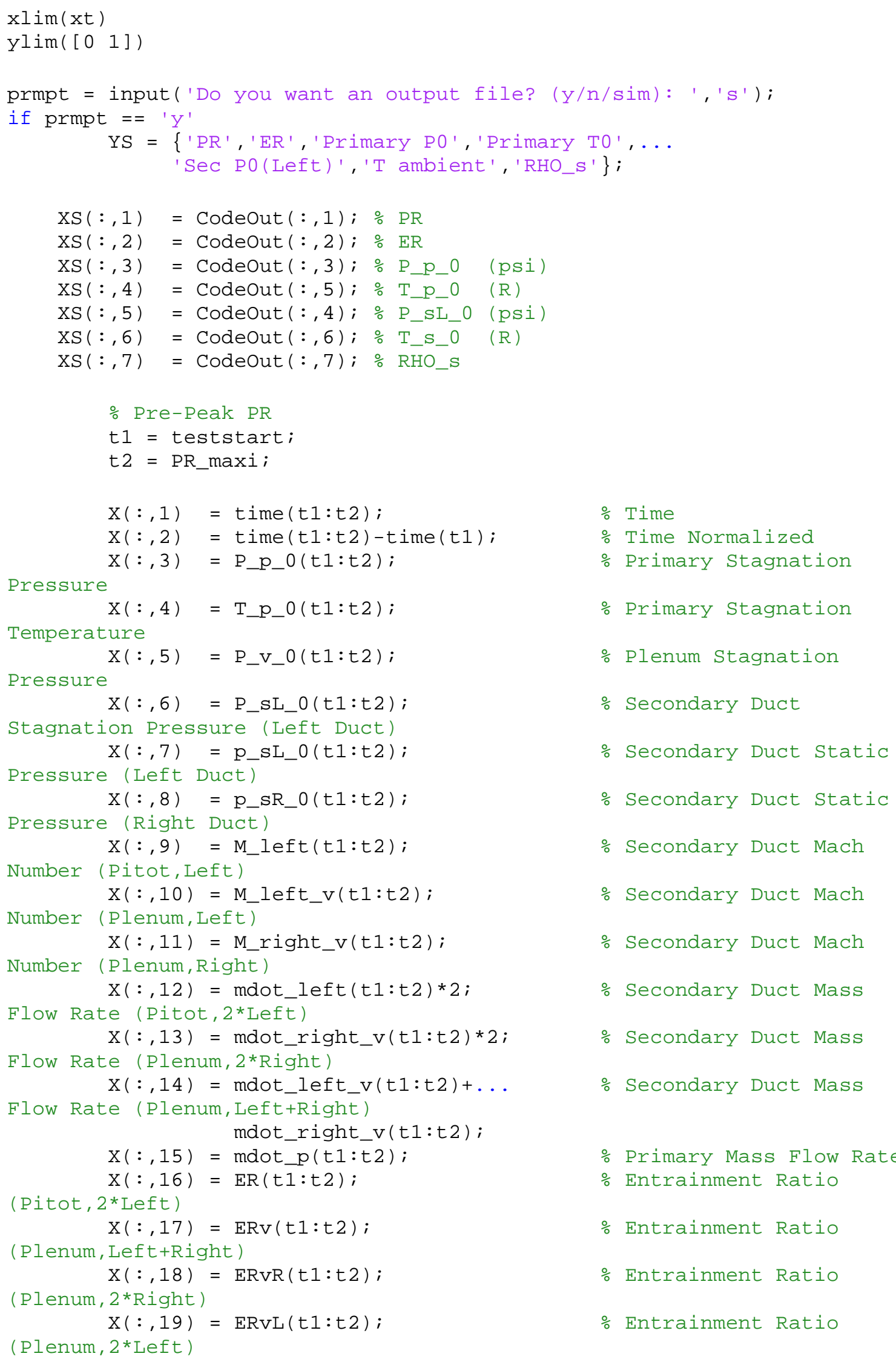




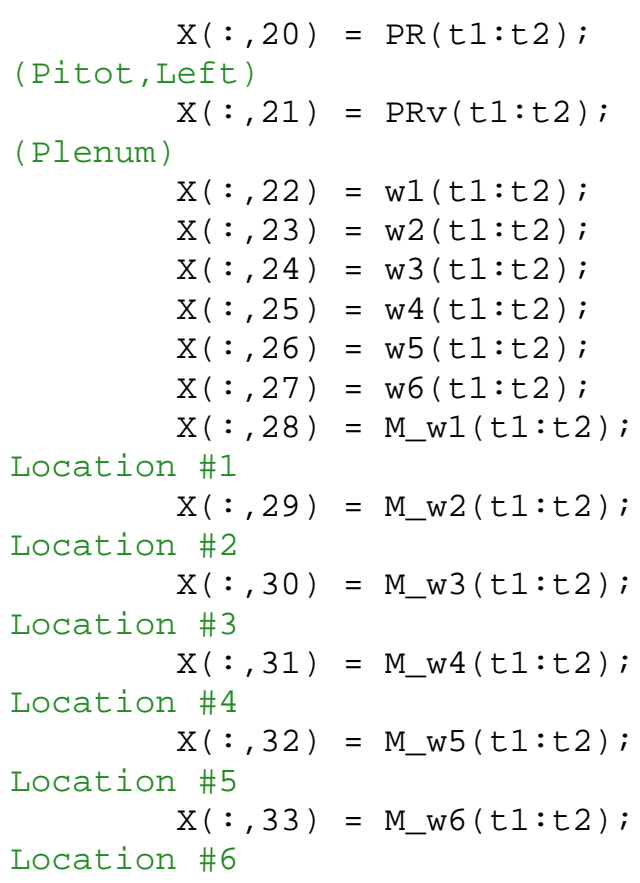

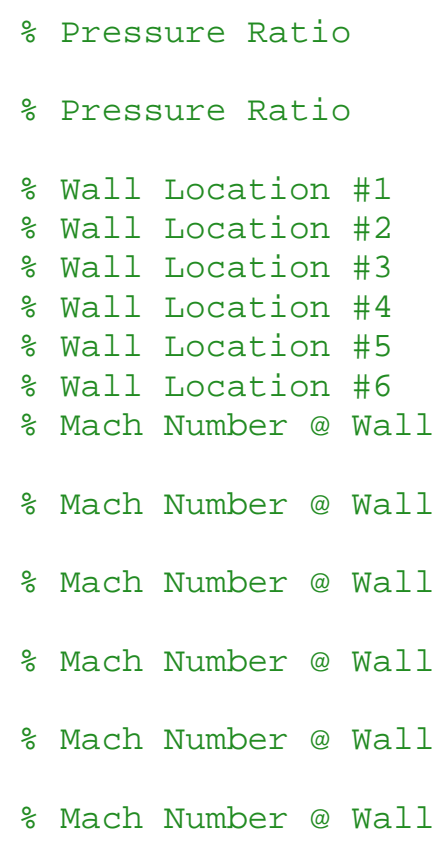




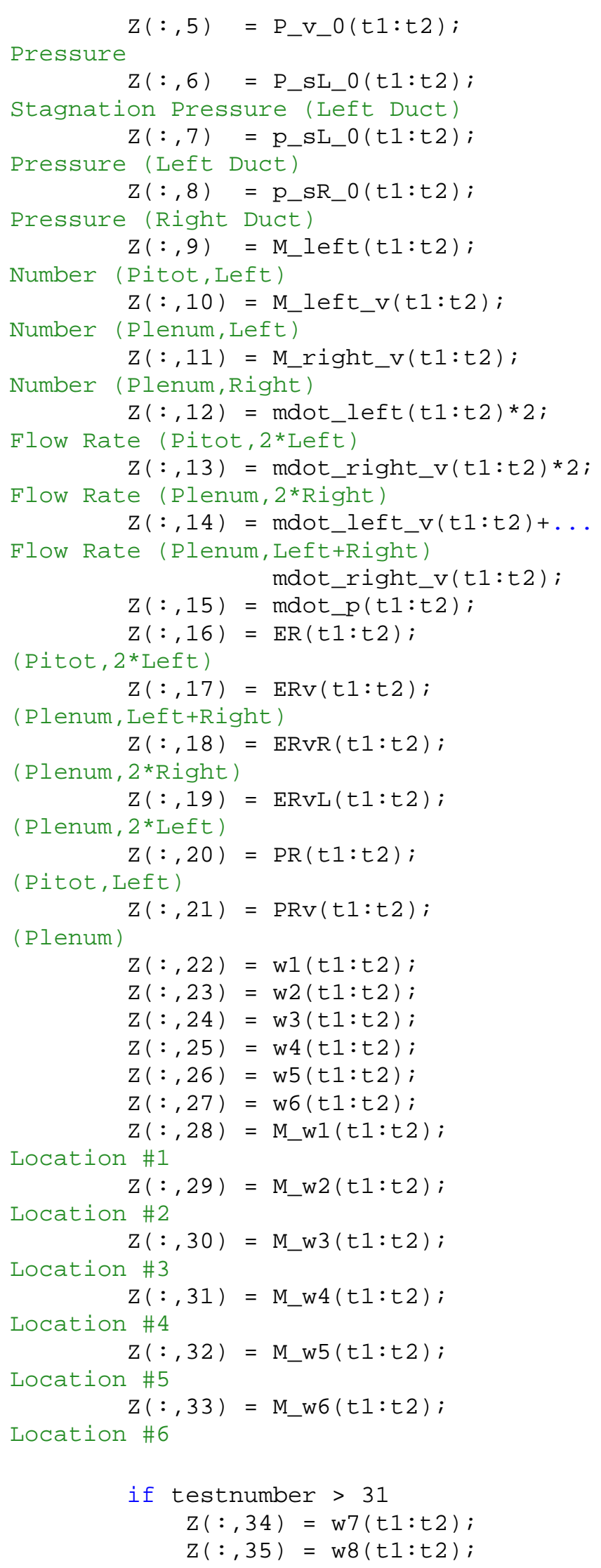

$\%$ Wall Location \#7

$\%$ wall Location \#8 


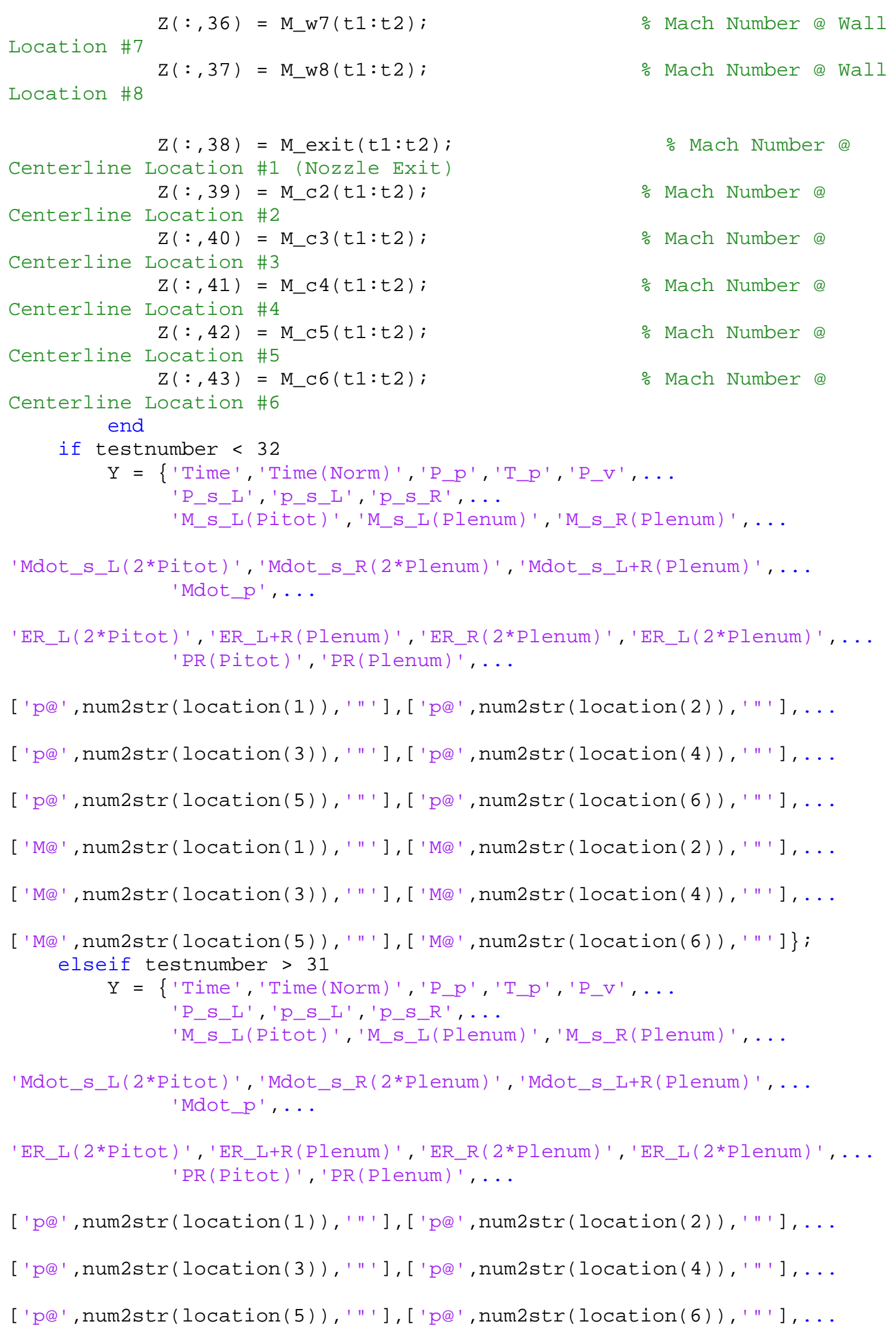


['M@ ', num2str(location(1)), '" '], ['M@ ', num2str(location(2)), ' " '], . . ['M@' , num2str(location(3)), '"'], ['M@' , num2str(location(4)), '"' ], . . ['M@', num2str(location(5)), '"'], ['M@ ', num2str(location(6) ), '" '], . . ['p@', num2str(location(7)), '"'], ['p@', num2str(location(8)), '"'], . . ['M@', num2str(location(7)), '"' ], ['M@ ', num2str(location(8) ), '" '], . . ['Mc@',num2str(locationc(1)),'"' ], ['Mc@',num2str(locationc(2) ), ' " '], . . ['Mc@', num2str(locationc(3)), '"'], ['Mc@', num2str(locationc(4)), '"'], . . ['Mc@',num2str(locationc(5)), ' " '], ['Mc@' ,num2str(locationc(6)), ' " ']\}; end

xlswrite(['output ', num2str(testnumber), '.xls'],YS, 'Code Input Titles');

xlswrite(['output ', num2str(testnumber), '.xls'],XS, 'Code Input Data'); xlswrite(['output ', num2str(testnumber), '.xls'],Y, 'Titles'); xlswrite(['output ', num2str(testnumber), '.xls'], X, 'Data (PrePeak)' );

xlswrite(['output ', num2str(testnumber), '.xls'],Z, 'Data (PostPeak )' );

end 